\title{
MASTER
}

COO-119-233

\section{Research in Radiobiology}

\author{
Semi-Annual Report of Progress \\ in the Internal Irradiation Program \\ of the
}

RADIOBIOLOGY DIVISION OF THE DEPARTMENT OF ANATOMY

UNIVERSITY OF UTAH COLLEGE OF MEDICINE

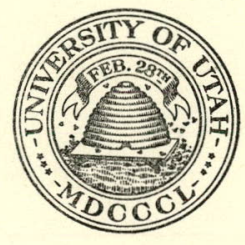

Respectfully Submitted by

THOMAS F. DOUGHERTY, Director

SEPTEMBER 30, 1965

RELEASED FOR ANNOUNCEMENT

IN NUCLEAR SCIENCE AESTRACTS

CONTRACT NO. AT (11-1)-119 


\section{DISCLAIMER}

This report was prepared as an account of work sponsored by an agency of the United States Government. Neither the United States Government nor any agency Thereof, nor any of their employees, makes any warranty, express or implied, or assumes any legal liability or responsibility for the accuracy, completeness, or usefulness of any information, apparatus, product, or process disclosed, or represents that its use would not infringe privately owned rights. Reference herein to any specific commercial product, process, or service by trade name, trademark, manufacturer, or otherwise does not necessarily constitute or imply its endorsement, recommendation, or favoring by the United States Government or any agency thereof. The views and opinions of authors expressed herein do not necessarily state or reflect those of the United States Government or any agency thereof. 


\section{DISCLAIMER}

Portions of this document may be illegible in electronic image products. Images are produced from the best available original document. 


\title{
RESEARCH IN RAD IOBIOLOGY
}

\author{
Semi-Annual Report of Work in Progress \\ in the Internal Irradiation Program
}

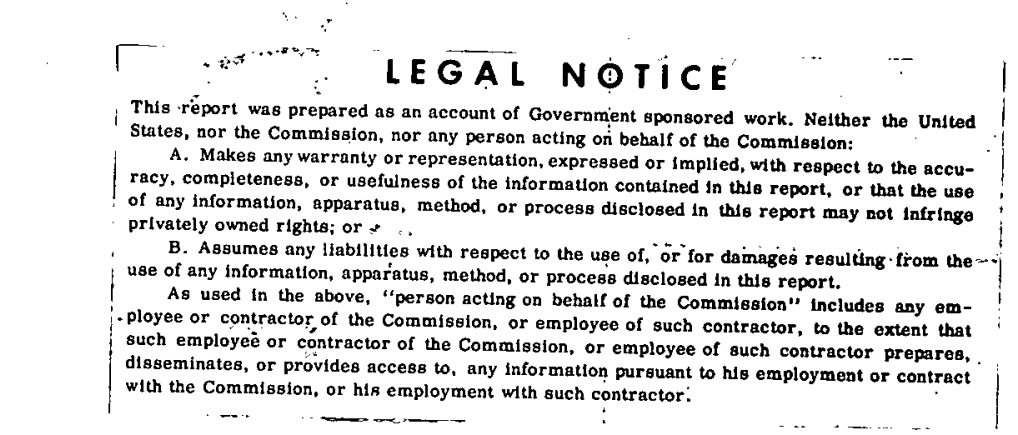

Radiobiology Division of the Department of Anatomy, University of Utah College of Medicine

Respectfully Submitted by:

Thomas F. Dougherty, Director

REIHASED FOR ANNOUNCEMENT

IN MUCLEAR SCIEMCE ABSTRACTS

September 30,1965

Contract No. AT (11-1)-119 
TABLE OF . CONTENTS

Page

Availability, of Previous Reports.

Group Leaders and Sections

Current Census of the Beagle Colony

Preface to Injection Tables

Dosimetry Revisions

Injection Tables

I. Toxicity Animals

A. Radium-226

10

B. Plutonium-239

18

C. Radium-228 (Mesothorium)

26

D. Thorium-228 (Radiothorium)

E. Strontium-90

II. Test Animals

A. Radium-226

44

B. Plutonium-239

50

C. Radium-228 (Mesothorium)

54

D. Thorium-228 (Radiothorium)

56

E. Strontium-90

58

F. Radium-224 (Quickradium)

$62^{\circ}$

G. Ancillary

Special Topics

An Analysis of Hematölogical Data from Beagles Given

$\mathrm{Ra}^{226}$ Using the Randomized Block Analys is of Variance. 68

Soft Tissue Retention of $\mathrm{Sr}^{90}$ in the Beagle.

Determination of $\mathrm{Sr}^{\circ}{ }^{\circ}$ in Biological Material with Solvent

Extraction of $\mathrm{Y}^{90}$ into Scintillator Solution.

Preparation of $\mathrm{Sr}^{90}$ Standard in Plastic Block for.

Densitometric Evaluation of Radioautograms.

Gamma-Ray Emitters in Concrete.

Use of the Chinchilla in the Study of Bone Seeking Radionuclides.

Radium Retention in Chinchillas. 
AVA ILABILITY OF PREVIOUS REPORTS

Copies of our reports may be obtained from the Office of Technical Services, U. S. Department of Commerce, Washington $25 ;$ D. C.

\begin{tabular}{|c|c|c|c|c|}
\hline Report & Date & Title & Photocopy & Microfilm \\
\hline$\overline{c 00-119-233^{*}}$ & Sep 1965 & Research in Radiobiology. & $\$ 10.90$ & $\$ 5.45$ \\
\hline C00-119-232* & Mar 1965 & Research in Radiobiology & 22.20 & $.11 \cdot 10$ \\
\hline Coo-119-231* & Sep 1964 & Research in Radiobiology & 17.20 & 8.60 \\
\hline $\operatorname{co0}-119-230^{*}$ & Jul 1964 & Safety Manual & 3.10 & 1.55 \\
\hline C00-119-229* & Mar 1964 & Research in Radiobiology & 13.00 & 5.84 \\
\hline $\operatorname{coo}-228^{*}$ & Sep 1963 & Research in Radiobiology. & 13.00 & 5.84 \\
\hline $\operatorname{coo}-227^{*}$ & Mar 1963 & Research in Radiobiology & 15.50 & 7.22 \\
\hline $\operatorname{co0}-226^{*}$ & Sep 1962 & Research in Radiobiology & 10.50 & 4.31 \\
\hline $\operatorname{co0}-225$ & Mar 1962 & Research in Radiobiology & 10.50 & 4.28 \\
\hline $\operatorname{co0}-224^{*}$ & Sep 1961 & Research in Radiobiology & 9.10 & 3.32 \\
\hline $\operatorname{co0}-223^{*}$ & Mar 1961 & Research in Radiobiology & 6.30 & 3.00 \\
\hline $\operatorname{coo}-222^{*}$ & Sep 1960 & Research in Radiobiology & 22.80 & 7.20 \\
\hline $\operatorname{coo}-221^{*}$ & Aug 1960 & Interim Report of $\mathrm{Sr}^{90}$ & 3.30 & 2.40 \\
\hline $\operatorname{coo}-220^{*}$ & Mar 1960 & Research in Radiobiology & .34 .80 & 9.90 \\
\hline $\cos -219^{*}$ & Sep 1959 & Semi-Annual Report & 10.80 & 3.90 \\
\hline $\operatorname{co0}-218^{*}$ & Mar 1959 & Annual Report & 34.80 & 9.90 \\
\hline AECU- 4112 & Feb 1959 & Radioactive Fallout & 3.30 & 2.40 \\
\hline $\operatorname{coo}-217^{*}$ & Sep 1958 & Semi-Annual Report & 28.80 & 8.40 \\
\hline $\cos -216^{*}$ & Mar 1958 & Escape of Radon \& Thoron & 9.30 & 6.30 \\
\hline $\cos -215^{*}$ & Mar 1958 & Annual Report & 28.80 & 8.40 \\
\hline AECU-3 3583 & Sep 1957 & Semi-Annual Report & 19.80 & 6.30 \\
\hline AECU-3522 & Mar 1957 & Annual Report & 27.30 & 8.10 \\
\hline TID-16459 & Sep 1956 & Semi-Annual Report & 5.60 & 2.05 \\
\hline TID-16458 & Mar 1956 & Annual Report & 10.50 & 4.40 \\
\hline AECU-3109 & Sep 1955 & Semi-Ánnual Report & 12.30 & 4.50 \\
\hline AECU-3418 & Mar 1955 & Annual Report & 9.30 & 3.60 \\
\hline TID-7639 & Consultan & ts Meeting & 19.75 & 9.40 \\
\hline
\end{tabular}


Division of Radiobiology, Department of Anatomy, University of Utah College of Medicine

\section{Section}

Director

Biochemistry

Bone

Chem istry

Clinic \& Pathology

Hematology

Physics

Radiology
Group Leader

Prof. Thomas F. Dougherty

Dr. David L. Berliner

Dr. Webster S. S. Jee

Dr. Betsy J. Stover

Dr. Glennn N. Taylor

Dr. Jean H. Dougherty

Dr. Charles W. Mays

Dr. William R. Christensen 
CURRENT CENSUS OF THE BEAGLE COLONY

September 30, 1965

Assignment

Number of Dogs

Plutonium, toxicity (Pu-239)

Plutonium, test (Pu-239)

Radium, toxicity (Ra-226)

Radium, test (Ra-226)

Quickradium, test $(\mathrm{Ra}-224)$

Radiothorium, toxicity (Th-228)

Radiothorium, test (Th-228)

Mesothorium, toxicity (Ra-228)

Mesothorium, test $(\mathrm{Ra}-228)$

Strontium, toxicity (Sr-90)

Strontium, test (Sr-90)

Controls, aging 45

Controls, $x-$ ray

Unassigned (Male 68, Female 64) 


\section{INJECTION TABLES}

Tables I and II list the toxicity and test animals, respectively. Toxicity animals are those animals which will be maintained until sacrifice becomes a clinical necessity; test animals may be sacrificed as needed for special studies.

Dogs are put into the toxicity study groups of six or seven of the same sex (five or six dose levels plus one control). Litter mates are used whenever possible. Each animal receives the designated dose of one radionuclide in a single I.V. injection. The animals are injected at approximately 17 months of age. At this age the skeleton is mature with all epiphyses fused except those of the ribs. Twelve such groups have been injected for each of the fivé radionuclides, $\mathrm{Ra}^{226}$, $\mathrm{Pu}^{239}, \mathrm{Ra}^{238}$, $\mathrm{Th}^{228}$, and $\mathrm{Sr}^{90}$. The current injection program is limited to $\mathrm{Ra}^{23 s}$ and $\mathrm{Pu}^{239}$ at lower dose levels.

The five dose levels designated by integers are those specified at the early meetings of the consultants, and those designated by nonintegers have been added by the laboratory staff. Since those dose levels were originally specified as "retained" doses, the actual. injected doses are four times the desired "retained". doses of $\mathrm{Ra}^{236}$, $\mathrm{Ra}^{228}$ (Mesothorium), and $\mathrm{Sr}^{\circ 0}$, and $\mathrm{l} .11$ times the desired "retained" doses of $\mathrm{Pu}^{239}$ and $\mathrm{Th}^{228}$ (Radiothorium).* The desired "retained" activities are the same for all

$*$

Since radioactive decay and excretion occur continuously, the term "retained" dose is obviously meaningless unless the time after injection is specified. Our present measurements indicate that

$\begin{array}{ll}\text { average } \mathrm{Ra}^{226} & \text { retention }=0.25 \text { after } 271 \text { days } \\ \text { average } \mathrm{Pu}^{239} & \text { retention }=0.90 \text { after } 6 \text { days } \\ \text { average } \mathrm{Ra}^{228} & \text { retention }=0.25 \text { after } 214 \text { days } \\ \text { average } \mathrm{Th}^{228} & \text { retention }=0.90 \text { after } 6 \text { days } \\ \text { average } \mathrm{Sr}^{90} & \text { retention }=0.25 \text { after } 135 \text { day.s }\end{array}$


the radionuclides except $S r^{\circ}$, in which case they are greater by a factor of 10. Dose level 1 , is the basis of the scheme, and is 10 times the maximum permissible $\mathrm{Ra}^{226}$ in man. Level $1=10 \times \frac{0.1 \mu \mathrm{C} \mathrm{Ra} 26}{70 \mathrm{~kg} \text { man }}=$ 0.01 .43 "retained" $\mu \mathrm{c} / \mathrm{kg}$. All other dose levels are simple multiples of level 1 as shown below.

$\begin{array}{lrlr}\text { Level } 0.1 & \text { is } 1 / 27 & \text { of level } 1 \\ \text { Level } 0.2 & \text { is } 1 / 9 & \text { of level } 1 \\ \text { Level } 0.5 \text { is } 1 / 3 & \text { of level } 1 \\ \text { Level } 1.5 & \text { is } & 2 \text { times level } 1 \\ \text { Level } 1.7 \text { is } & 3 \text { times level } 1 \\ \text { Level } 2 & \text { is } & 6 \text { times level } 1 \\ \text { Level } 3 & \text { is } 18 \text { t.imes level } 1 \\ \text { Level } 4 & \text { is } 54 \text { times level } 1 \\ \text { Level } 5 & \text { is } 162 \text { times level } 1\end{array}$

The numbering system for the dogs has been built around the injection program and serves as a code to describe each dog's place in: the experiment. The first letter tells the sex of toxicity animals $(M=$ male, $F=$ female). When the first letter is $T$, the dog is a test animal. $\mathrm{M}, \mathrm{F}$, or $\mathrm{T}$ is followed by a number which denotes chronological order of groups in the case of toxicity dogs and of individual test dogs.

Next comes a code letter for the radionuclide: $\mathrm{R}=\mathrm{Ra}^{226}, \mathrm{P}=\mathrm{Pu}^{239}$, $M=\mathrm{Ra}^{2.28}, \mathrm{~T}=\mathrm{Th}^{228}, \mathrm{~S}=\mathrm{Sr}^{90}, \mathrm{Q}=\mathrm{Ra}^{2.24}, \mathrm{~J}=\mathrm{Sr}^{85}$, and $\mathrm{A}=$ Aging Control.

"A" following the regular dog number means that the dog. is a replacement. " $\mathrm{H}$ " following the regular dog number means that the dog received its dose in more than one injection. " $\mathrm{B}$ ", "C", or " $\mathrm{D}$ " denotes assignment to serial sacrifice schedule. Any of the above letters denoting a radionuclide may follow the final number, in which case the letter indicates that two radionuclides were "given. The dose level refers to the radionuclide appearing first in the identifying code.

Example: MIR5 is a male animal in the first radium group at the highest dose level. 
'Althoügh MlR5,'MIR4; MIR3;, MIR2; MIRI, and MIRO constitute a group and were injected at the same time, the tables are arranged according to dose level to facilitate comparison of all the R5 animals, all the R4 animals, etc.

The conditions listed in the injection tables under "Comments on Dead Dogs" present the lesions or factors that had the most prominent effect on the clinical status of the animal. For example, multiple rib fractures, which seldom produced symptoms, are not listed, even though their incidence was usually much higher than the crippling fractures involving thë limb bones or mandiblé. The hematological changes have been omitted unless they were extreme. Increased rate of tooth loss, hepatic changes, eye lesions, and many other factors in the various syndromes have not been included because of "space limitations. Over the years many soft tissue tumors have been removed surgically; these tumors were the subject of a separate report, Research in Radiobiology, September 30, 1963 (C00-228), pp. 95-108. In many instances, the conditions that have been listed were the reasons for sacrifice of the animal but they were not the immediate cause of death. Most of the animals were euthanized when death appeared eminent or when life could no longer be humanely prolonged.

\section{DOS IMETRY REVISIONS}

A number of new values appear for the dose in rads to the skeleton at death. $\mathrm{Ra}^{226}, \mathrm{Ra}^{228}$, and $\mathrm{Sr}^{90}$ doses are calculated for each dog using his individually observed retention values: $\mathrm{Pu}^{239}$ and $\mathrm{Th}^{228}$ doses ar̉e from öur average skeletal retention equations. 
For our standard beagle, the following equations, were used for the effective* skeletal retention at ( $t$ ) days after injection:

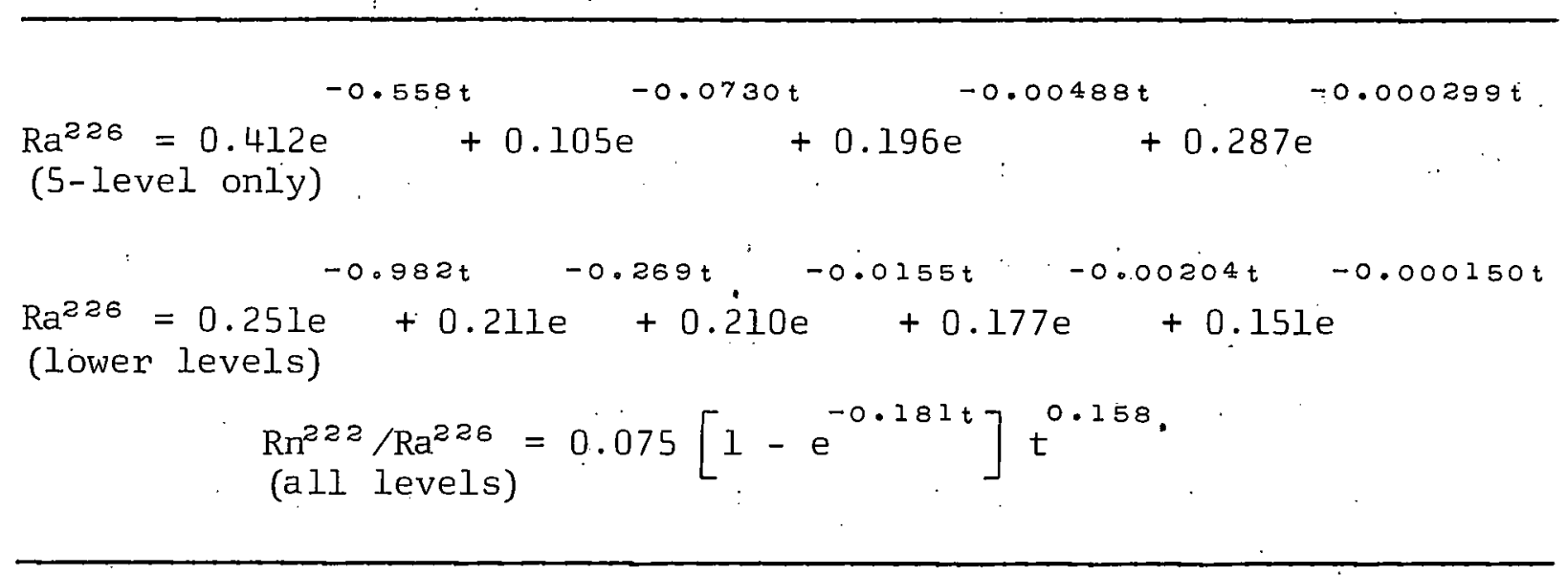
$\mathrm{Pu}^{239^{\prime}}=0.72 \mathrm{t}^{-0.040}$

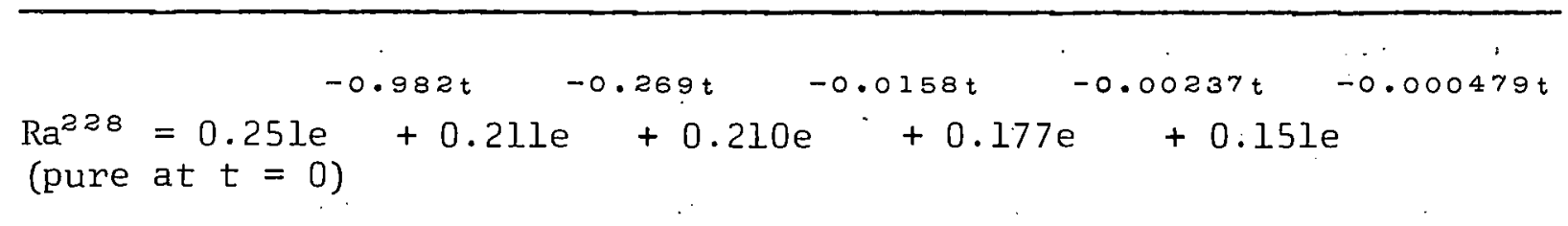

$84.4 \%$ retention of in vivo produced $\mathrm{Th}^{228}$ and daughters.

$$
\begin{aligned}
\mathrm{Th}^{228} & =0.69 \mathrm{e}^{-0.00113 t} \\
\mathrm{Ra}^{224} / \mathrm{Th}^{228} & =0.895 \\
\mathrm{~Pb}^{212} / \mathrm{Th}^{228} & =0.866
\end{aligned}
$$

$S r^{90}=0.402 e^{-0.748 t}+0.259 e^{-0.102 t}+0.181 e^{-0.00469 t}+0.158 e^{-0.000302 t}$.

*Effective retention is decreased by both radioactive decay and biological elimination. 
We hope to present our detailed retention data and dosimetric analysis in a future report.

$\mathrm{Ra}^{228}$ doses deserve special comment. The dose from "pure" $\mathrm{Ra}^{238}$

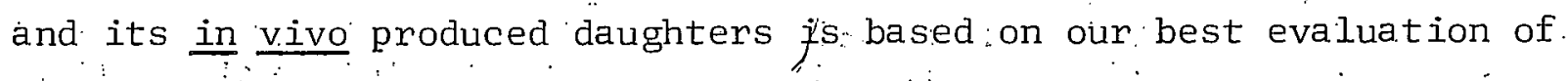
$5.77 \pm 0.02 \mathrm{yr}$ for the $\mathrm{Ra}^{238}$ half-period. The tabulated total doses include the contributions from. Th ${ }^{238}$ contamination in the injection solutions. For example, $\mathrm{Th}^{2} 28$ contaminations of $0.6 \%, 3 \%$, and $15 \%$, respectively; account for $2.8 \%, .13 \%$ and $42 \%$ of the total dose in rads at 1000 days: If injected $\operatorname{Th}^{228}$ is 4 times more toxic rad-for-rad than is in vivo produceed $\mathrm{Th}^{228}$, these injected $\mathrm{Th}^{228}$ contaminations would account for $10 \%, 37 \%$ and: $74 \%$ of the total biological damage at 1000 days. Therefore, it may be desirable to use only results from the slightly. contaminated $\left(0.6 \% \mathrm{Th}^{228}\right)$ dogs in evaluation of $\mathrm{Ra}^{228}$. toxicity. 
TABLE I. TOXICITY ANIMALS (SEPT.30, 1955)

A. RADIUM-225

DOG

NUMBER

MOIRO.O

FO3RO.0

MOR O.0

MOSR.O. 0

FCGR 0.0

$M 07 R 0.0$

FOSR 0.0

FO SR 0.0

M IORO:O

F11RO.O

F12RO.0

il $13 R 0.0$

FI 4 R 0.0

MISPC.O

F16R0.0

F3IRO.OB

F3IRO.OC

F31R O.OD

$F 32 P O .0 B$

F $32 R 0.0 C$

F32R O.OD

F3 $3 R 0.0 B$
AT INJECTION

AGE hEIGHT

(DAYS) (KG)

558

487

$6 C 1$

461

460

483

511

638

$7 \mathrm{CO}$

522

544

50.1

515

536

564

469

536

536

542.

542

532

532

532
8.03

14.6

11.4

11.0

6.57

8.43

11.0

8. 21

11.7

10.9

1C. 2

8.68

12.3

10.8

12.8

10.0

10.6

9.88

9.9

7.8

$11 . ?$

5. 7

9.8
DATE

IN JEC TED

$(\mu \mathrm{C} / K G)$
DAYS SINCE

IN JECTED

D MO YR 3J/9/65 DEATH

$20 \quad 04 \quad 53$

151153

100354

$\begin{array}{lll}7 & 0454\end{array}$

$\begin{array}{lll}22 & 05 & 54\end{array}$

27754

$24 \quad 354$

$21 \quad 12 \quad 54$

11455

270755

$20 \quad 12 \quad 55$

170156

4354

$2.310 \quad 54$

4255

$7+55$

231254

$\begin{array}{lll}23 & 10 & 54\end{array}$

21955

21955

$21 \quad 955$

21955

21935
3116

3675

2139

4194

4018

3182

3360

3361

1550
3718

3572

3544

575

342

238

176

342

342

9

9

9

9

9

F $13 R 0.2$

F1 $14 R 0.2$

$15 R 0.2$

529

9.77

.00577

4. 354

575

8.10

.00836

$2310 \quad 54$

34 ?

EC4 $1 \mathrm{C.8}$

.00873

4255

$23 t$

8.90

. 00665

74.55

176

M $13 R .0 .5$

F 1480.5

M1 $15 R 0.5$

FIERO.5

529

510

11.0

9.75

$400 \quad 10.4$

$500 \quad 11.4$

.0171

4354

575

$\begin{array}{lll}23 & 10 & 54\end{array}$

342

4255

238

.0263

7455

176

MOLR1.0

MO2R I. O

FO 3R 1.0
471

8.48

627

706
10.0

8. 68
.0618

.0876

.0576
200453

$\begin{array}{lll}15 & 11 & 53\end{array}$

$10 \quad 03 \quad 54$
4546

4054

3850
DOSE TO

SKELETCN

(RAOS) 
DOG

NUMBER

MOLRO.O MOZRO. 0 F.03R 0.0 MOLRO.O MOSR 0.0 FO GR 0.0 MOTR O.O FO $8 R 0.0$ FOSRO.O MIOR O. 0 FIIRO.0 FI $12 R 0.0$ M I 3R 0. 0 F1 1 RR 0.0 M15R0.0 F 1 6R 0.0 F $31 R 0.0 B$ F31RO.OC F $31 R 0.0 D$ F32RO.OB F $32 R 0.0 \mathrm{C}$ F32R O.OO F 33R 0.OB

$M 13 R 0.2$ FI $4 R 0.2$ M15R0.2 FI6RO. 2

M1 3R 0.5 F1 4R0.5 M 1 5R 0.5 FI $6 R 0.5$

MOLR I.O MO2R 1.0 FO $3 R 1.0$
SEM INOMA, LYMPHOSARCOMA

TRANSITIONAL CELL. CARCINOMA

STATUS EPILEPTICUS

OBTURATING PULMONARY EMBOLISM

STATUS EPILEPTICUS

STATUS EPILEPTICUS, NEPHRITIS

PANCREATIC ADENOCARCINOMA AGR TIC BODY TUMOR 


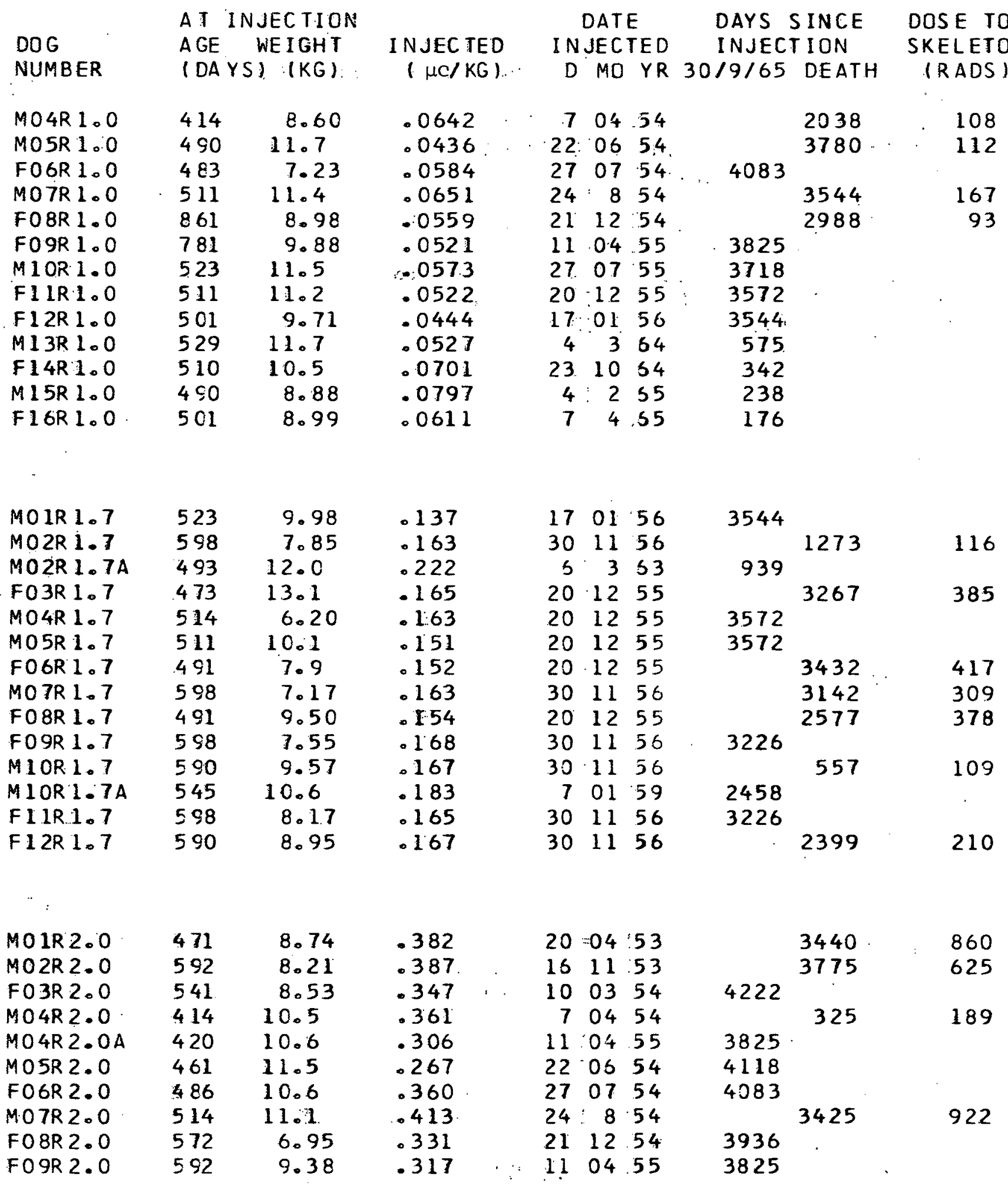


DOG

NUMBER COMMENTS ON DEAD DOGS

MO4R 1.0 MOSR 1.0 FO6R 1.0 MOTR 1.0 FO 8R 1.0 FOSR 1.0 M1OR 1.0 FIIR 1.0 F I 2R 1.0 $M 13 R 1.0$ F14R1.0 MI IR I.O

F16R1。0

$M 01 R 1.7$ MO2R 1. 7 MO2R 1.7A FO3R 1.7 $M 04 R 1.7$ MOSR 1.7 FO6R 1.7 MOTR 1.7 FOQR 1.7 FO9R 1.7 MIOR 1.7 MIOR 1.7A FIIR 1.7 FI 2R 1.7

L YMPHOSARCOMA

MAMMARY GLAND CARCINOMA

STA TUS EPILEPTICUS

L YM.PHOSARCOMA

TRA UMA

TRANSITIONAL CELL CARCINOMA,HYDRONEPHROSIS

BAC TERIAL TOXEMIA INTERSTITIAL CELL CARCINOMA DRUG ALLERGY

TRA UMA

MOIR2.0

HEMANGIOSARCOMA

$M O 2 R 2.0$ OSTEUSARCOMA

FO3R 2。0

$M O 4 R 2.0$

PERFORATED I LEUM

$M 04 R 2.0 A$

MOSR 2. 0

F06R 2.0

MOTR2.0

FO 8R 2.O

OSTEOSARCOMA, CUSHING SYNDROME

FO $9 R \cdot 2.0$ 
DOG

NUMBER

M1OR2.0

FIIR2.0

FI2R2.0

MOIR3.0

MO 2R 3.0

F0 3R 3.0

MO $4 R 3.0$

MOSR 3.0

FO6R 3.0

MOTR 3.0

FO BR 3.0

FO 9R 3.0

$M 1 O R 3.0$

FI 1R 3.0

F12R 3.0
A T INJECTION AGE WEIGHT (DAYS) (KG)

\section{3}

495

497
IN JEC TED

$(\mu \mathrm{C} / \mathrm{KG})$
DATE

IN JECTED

DAYS SINCE INJECT IDN

D MO YR 30/9/65 DEATH

$2707 \quad 55$

$\begin{array}{lll}20 & 12 & 55\end{array}$

17156

200453

15 11.53

$10: 0354$

$\begin{array}{lll}7 & 04.54\end{array}$

$2205 \quad 54$

$2707 \quad 54$

240854

21.12 .54

$1104: 55$

$\begin{array}{lll}27 & 07 & 55\end{array}$

$20 \cdot 1255$

17156
3569

3297

2948
DOSE TO

SKELETON

(RADS)

1220

728

742

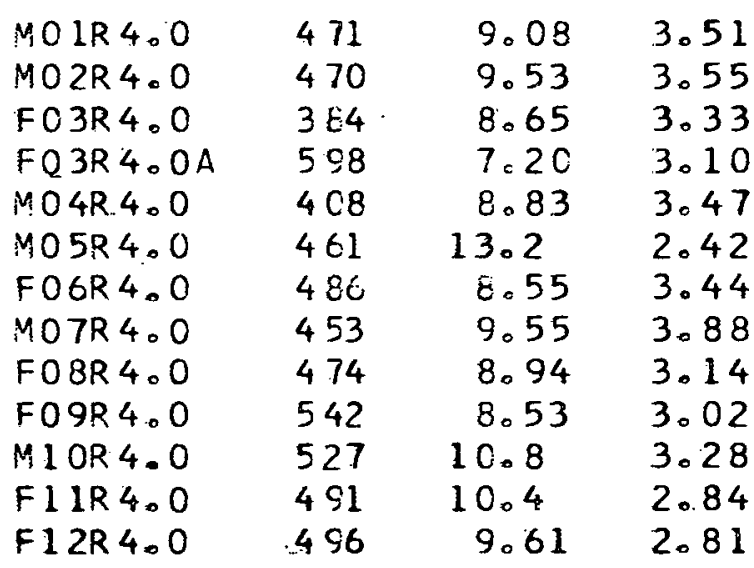

2395

1727

2323

2361

2317

2246

3029

1916

1839

2336

1333

1639

$\begin{array}{ll}\text { MOIR 5.0 } & 473 \\ \text { MO2R 5.0 } & 470 \\ \text { FO 3R 5.0 } & 380 \\ \text { MO4R 5.0 } & 4 C 8 \\ \text { MOSR 5.0 } & 458 \\ \text { FO 6R 5.0 } & 486\end{array}$

$\begin{array}{cc}9.87 & 10.5 \\ 8.85 & 10.8 \\ 7.82 & 10.1 \\ 8.90 & 10.6 \\ 10.9 & 10.1 \\ 9.66 & 10.2\end{array}$

$\begin{array}{rrr}20 & 04 & 53 \\ 16 & 11 & 53 \\ 10 & 03 & 54 \\ 30 & 11 & 56 \\ 7 & 04 & 54 \\ 22 & 05 & 54 \\ 27 & 07 & 54 \\ 24 & 8 & 54 \\ 21 & 12 & 54 \\ 11 & 04 & 55 \\ 27 & 07 & 55 \\ 20 & 12 & 55 \\ 17 & 01 & 56\end{array}$

1606

1834

490

1614

1518

1659

1939

1647

1324

147.1

1553

1469

1435
$20: 0.453$

908

1380

481

$10: 03 \quad 54$

7: $04: 54$

1091

1220

1015

6575
6150
2208
3855
6063
4505
7133
5844
4615
4095
7582
5273
3877

$\begin{array}{rrr}22 & 6 & 54 \\ 27 & 07 & 54\end{array}$
14943

18071

7147

16417

15433

15414 
DOG

NUMBER

M 1OR2.0

FIIR2.0

O STEOSARCOMA

F1 2R2.0

MAMMARY ADENOCARCINOMA.

MOLR 3.0

MO2R3.O

FO 3R 3.0

MO4R 3.0

MOSR 3.0

FO6R 3.0

MOTR3. 3.0

FO 8R 3.0

FOSR 3.0

M1OR 3.0

F11R 3.0

F1 $12 R 3.0$
DSTEOSARCOMA

O STEOSARCOMA

O STEOSARCOMA

O STEOSARCOMA

OSTEOSARCOMA

D STEOSARCOMA

O STEOSARCONA

OSTEOSARCOMA

OSTEOSARCOMA

OSTEOSARCOMA

P YOMETRITIS + SECONDARY PERITONITIS

O STEOSARCOMA

CONMENTS ON DEAD DOGS
$M 01 R 4.0$
O STEOSARCONA
MO2R 4.0
O STEOSARCOMA
FO 3R 4.0
CAN INE DISTEMPER
F.03R 4.0A
O STEOSARCOMA
$M 04 R 4.0$ :
O STEO SARCOMA
MOSR 4.0
O STEOSARCOMA
FO6R 4.0
OSTEOSARCOMA
MOTR 4.0
OSTEOSARCOMA.
FO 8R.4.0
O STEO SARCOMA
FOSR $4: 0$
OSTEOSARCOMA
M1OR 4.0.
O STEOSARCOMA
F1 1R.4.0
O STEOSARCOMA
F $12 R 4: 0$
O STEOSARCOMA

MOIR 5.0

O STEOSARCOMA

$M O 2 R 5.0$

FO 3R 5.0

$M O 4 R 5.0$

O STEOSARCOMA

CANINE DISTEMPER

MO 5R.5.0

- STEOSARCOMA

OSTEOSARCOMA

FO 6R 5.0

O STEOSARCOMA 


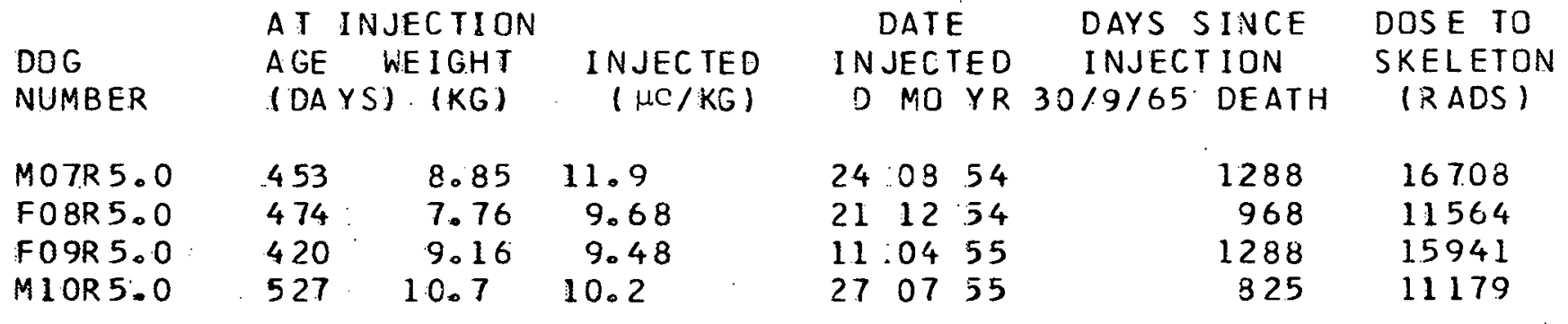


DOG

NUMBER

MOTR 5.0

FO 8R 5.0

FO $9 R 5.0$

MIOR 5.0

\section{COMMENTS ON DEAD DOGS}

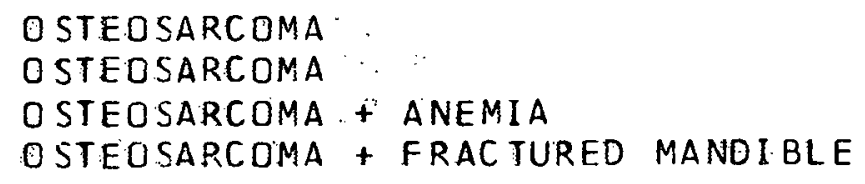


B. PLUTONIUM $\div 239$

\begin{tabular}{|c|c|c|c|c|c|c|c|c|}
\hline \multirow{3}{*}{$\begin{array}{l}\text { DOG } \\
\text { NUMBER }\end{array}$} & \multicolumn{2}{|c|}{ AT INJECTION } & \multirow{3}{*}{$\begin{array}{l}\text { I NJEC TED } \\
:(\mu \mathrm{K} / \mathrm{KG})\end{array}$} & \multicolumn{3}{|c|}{ DATE } & \multicolumn{2}{|c|}{ SINCE } \\
\hline & & & & I $N$ & JEC T & TED & INJECT & IION \\
\hline & (DAYS.) & $1(K G)$ & & $D$ & MO & $Y R$ & $30 / 9 / 65$ & DEAT! \\
\hline MOLPO.O & 443 & 9.70 & & 1 & 12 & 52 & & 400 \\
\hline$F 02 P 0.0$ & 424 & 6.36 & & 2 & 03 & 5.3 & & 275 \\
\hline$M 03 P 0.0$ & 515 & 10.8 & & 1 & 05 . & 53. & 4504 & \\
\hline M04P O.O & 426 & 10.7 & & 15 & 09 & 53 & 4397 & \\
\hline FOSP 0.0 & 620 & 9.75 & & 14 & 10 & 53 & & 4088 \\
\hline FO GP O.O & 410 & 5.59 & & 12 & 05 & 54 & 4159 & \\
\hline FOTP 0.0 & 515 & 6.90 & & 25 & 10 & .54 & 3993 & \\
\hline MO 8 P O.O & 585 & 10.9 & & 15 & 03 & 55 & 3852 & \\
\hline FO SP 0.0 & 658 & 11.0 & s & 2.2 & 11 & 55 & & 3032 \\
\hline FIOP 0.0 & 658 & 11.0 & & 22 & 11 & 55 & 3600 & \\
\hline M11P0.0 & 602 & 10.3 & & 24 & 04 & 56 & 3446 & \\
\hline$M 12 P 0.0$ & 630 & 10.9 & & 29 & 05 & 56 & 3411 & \\
\hline$F 13 P 0.0$ & 517 & 9.47 & & 4 & 3 & 54 & 575 & \\
\hline F14PO.0 & 452 & 9.89 & & 12 & 5 & 54 & 506 & \\
\hline MI $15 P 0.0$ & 527 & 12.1 & & 23 & 10 & 54 & 342 & \\
\hline M16P0.0 & 485 & 13.9 & & 7 & 4 & 55 & 176 & \\
\hline$M 31 P 0.0 B$ & 452 & 11.8 & & 12 & 5 & 54 & 506 & \\
\hline$M 31 P 0.0 C$ & 452 & 12.6 & & 12 & 5 & 64 & 506 & \\
\hline M32P0.0B & 452 & 11.2 & & 12 & 5 & 64 & 506 & \\
\hline$M 32 P 0.0 C$ & 542 & 10.3 & ' & 21 & 9 & 55 & 9 & \\
\hline$M 33 P 0.0 B$ & 517 & 12.1 & & 21 & 9 & 55 & 9 & \\
\hline
\end{tabular}

$\begin{array}{lcccrrrr}\text { F13P0.1 } & 515 & 9.46 & .00068 & 4: 3 & 54: & 575 \\ \text { F14P.0.1 } & 452 & 10.3 & .00055 & 12 & 554 & 506 \\ \text { M15P0.1 } & 536 & 9.67 & .00071 & 23 & 10 & 34 & 342 \\ \text { M16P0.1 } & 501 & 12.0 & .00059 & 7 & 4 & 55 & 176 \\ \text { M31P0.1B } & 517 & 12.2 & .00068 & 4: 3 & 54 & 575\end{array}$

\begin{tabular}{|c|c|c|c|c|c|c|c|}
\hline$F 13 P 0.2$ & 517 & 9.44 & .00206 & 4 & 3 & 54 & 575 \\
\hline F14P0.2 & 516 & 7.44 & .00173 & 12 & 5 & 64 & $\begin{array}{l}506 \\
342\end{array}$ \\
\hline M15P0.2 & 505 & 10.9 & .00201 & 23 & 10 & .34 & 342 \\
\hline M16P0.2 & 500 & 11.4 & .00163 & 7 & 4 & 55 & \\
\hline$M 31 P 0.2 B$ & 515 & 10.7 & .00185 & 4 & 3 & 54 & 57 \\
\hline $\mathrm{F} 31 \mathrm{PO} 0.2 \mathrm{C}$ & 452 & 11.9 & .00169 & 12 & 5 & 64 & 0 \\
\hline$F 31 P 0.2 n$ & 429 & 9.35 & .00186 & 12 & 5 & 64 & \\
\hline F $32 P 0.2 C$ & 494 & 10.1 & .00183 & 4 & 2 & 35 & \\
\hline$F 32 P 0.20$ & 490 & 8.04 & .00193 & $4_{4}$ & 2 & 55 & \\
\hline
\end{tabular}


DOG

NUMBER

COMMENTS ON DEAD DOGS

MOLP O.:O F02P 0.0 M03P0.0 MO4PO.0 F0 $5 P 0.0$ FO6P0.0 FO $7 P 0.0$ MO 8 P O.0 FO 9 P. 0.0 FIOP 0.0 M11P0.0 $M 12 P 0.0$ F13P0.0 FI 4P. 0.0 M15P0.0 MI 6P0.0 M31P0.OB M31PO.OC $M 32 P 0.0 B$ M32P0.OC M33P0.0B

F13P0.1 F1 $4 P 0.1$ M15P0.1 M16P0.1 M31PO.1B

F13P0.2 FI $4 P 0.2$ M15P0.2 $M 16 P 0.2$ M31P0.2B F. $31 P 0.2 C$ F31P0.2D F $32 \mathrm{PO} 0.2 \mathrm{C}$ F $32 P 0.2 D$

F13P0.5
SPLENIC RUPTURE, METASTATIC SEMINOMA ANE S THE TIC ACCIDENT

ADRENAL CORTICAL CARCI NOMA

PULMONARY EMBOLISM, NEPHRITIS 


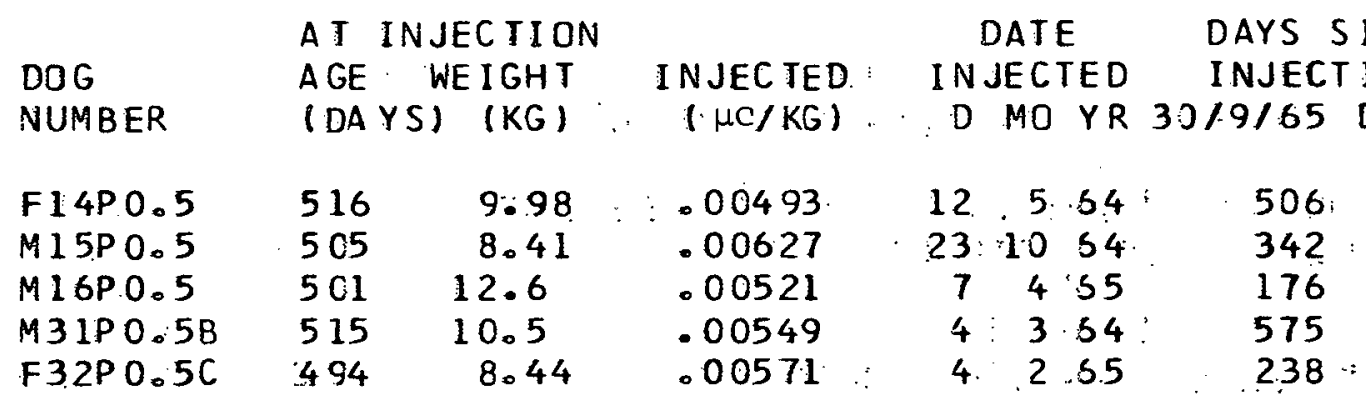

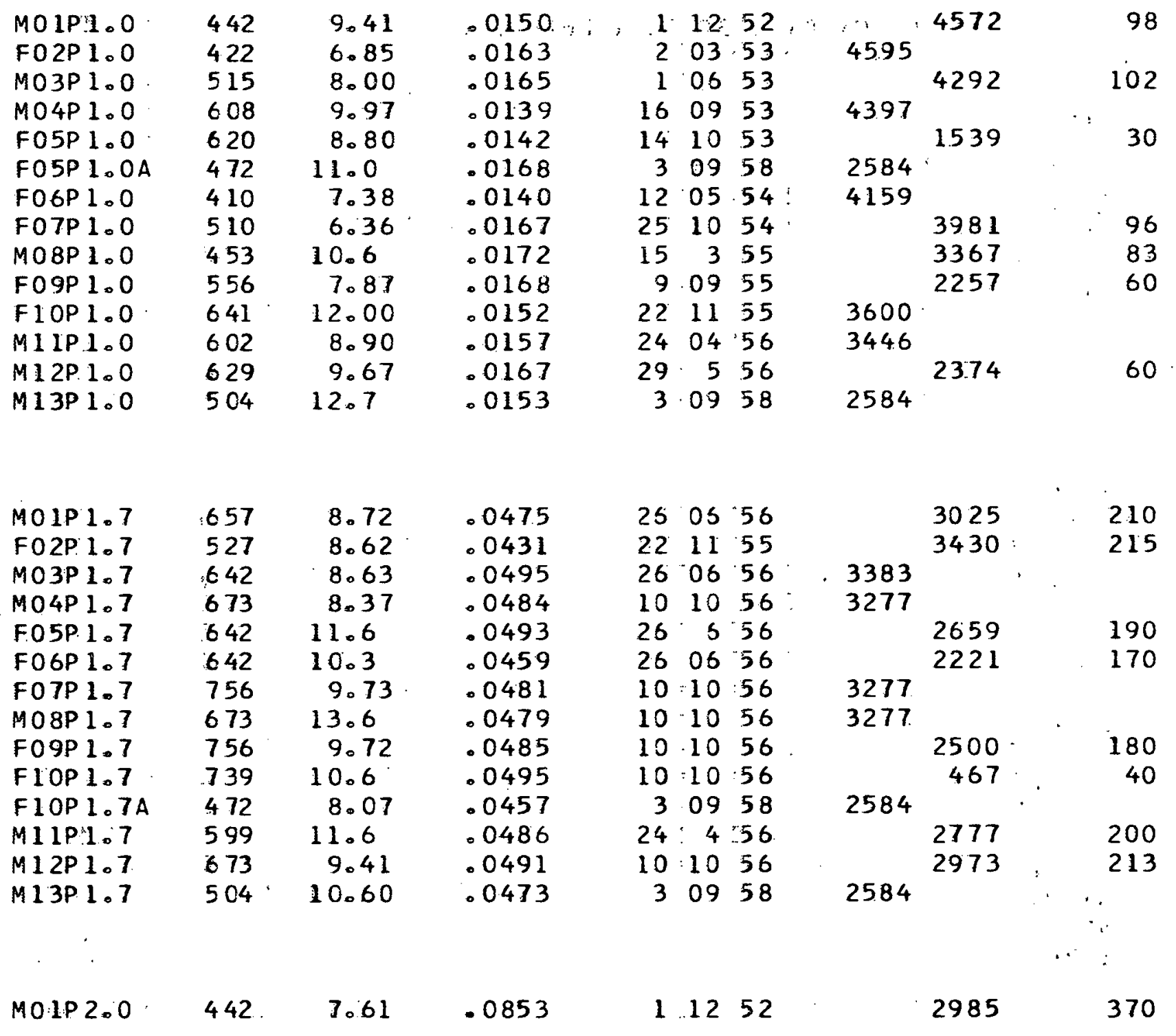


DOG

NUMBER

F1 4P. 0.5

M15P0.5

$M 16 P 0.5$

$M 31 P 0.5 B$

F32P0.5C

MO1P1.0

OSTEOSARCOMA

F02P 1.0

MO3P 1.0

O STEOSARCOMA

MO4P1.0

F05P 1.0

COLITIS, ENTERITIS + SECONDARY HEPATIC NECROS IS

F05P 1.0A

FO SP 1.0

FO7P 1.0

MO8P 1.0

TRA UMA LYMPHADENOPHATHY

F0 9P 1.0

O STEOSARCOMA

F1OP 1.0

M11P1.0

$M 12 P 1.0$

$M 13 P 1.0$

O STEOSARCOMA

CHRONIC PANCREATITIS

MO1P 1.7

FO2P 1.7

O STEOSARCOMA

MO3P 1.7

MO 4P 1.7

FO5P1.7

FO6P1.7

F07P 1.7

M08P 1.7

F09P 1.7

F10P1.7

OSTEOSARCOMA

F1OP1.7A

ACUTE ENTERITIS

$M 11 P: 1.7$

M12P1.7

O STEOSARCOMA

O STEOSARCOMA

M13P1.7

-BILE DUCT CARCINOMA

LEUKEMIA

MO1P2.0: OSTEOSARCOMA 
DOG

NUMBER

FO 2P. 2.0

M03P 2.0

MO4P. 2. 0

F05P 2.0

F0 6P 2.0

FO TP 2.0

MO 8P 2:0

FO 9P 2.0

F1OP2.0

M11P2.0

$M 12 P 2.0$
AT INJECTION AGE WEIGHT (DAYS) (KG)

422

485

608

594

417

485

406

552

551

599

622

$$
\text { 7. } 73
$$

10.5

9.84

8.12

7.54

8.40

9.73

9.72

7. 94

10.3

9.98
DATE

I NJEC TED

$(, \mu C / K G)$
INJECTED

D MO YR

20353

10553

$\begin{array}{lll}16 & 09 & 53\end{array}$

$14: 1053$

$1205 \quad 54$

$\begin{array}{lll}25 & 10.54\end{array}$

$\begin{array}{lll}15 & 03 & 55\end{array}$

9.0955

221155

24:04:56

2905.56
DAYS SINCE INJECTION

30/9/65 DEATH
DOSE TO SKELETDN

( $R$ ADS )

$\begin{array}{ll}2780 & 460 \\ 3185 & 420 \\ 2948 & 380 \\ 2423 & 310 \\ 2947 & 370 \\ 2093 & 310 \\ 1761 & 250 \\ 2014 & 300 \\ 2912 & 410 \\ 1617 & 230 \\ 2284 & 370\end{array}$

1476

1947

1604

1950

1504

1617

1627

177.1

1894

1547

1198

1659
580

900

700

840

630

670

750

780

840

700

550

760

$\begin{array}{llll}\text { MO1P4.0 } & 442 & 7.61 & .823 \\ \text { F02P4.0 } & 567 & 8.65 & 1.03 \\ \text { MO3P4.0 } & 485 & 9.36 & .929 \\ \text { MO4P4.0 } & 566 & 8.74 & .974 \\ \text { F05P4.0 } & 650 & 7.05 & .872 \\ \text { FO6P4.0 } & 420 & 9.26 & .811 \\ \text { FO 7P4.0 } & 485 & 8.45 & .963 \\ \text { MO8P4.0 } & 651 & 9.22 & .887 \\ \text { FO9P4.0 } & 552 & 8.58 & .960 \\ \text { F1OP4.0 } & 527 & 6.48 & .868 \\ \text { M11P4.0 } & 596 & 9.56 & .927 \\ \text { M12P4.0 } & 598 & 11.4 & .838\end{array}$

112.52

$\begin{array}{lll}2 & 03 & 53\end{array}$

10653

$\begin{array}{lll}16 & 09 & 53\end{array}$

141053

$1205 \quad 54$

2510.54

15.03 .55

90955

$22 \quad 1155$

$24: 04: 56$

2905.56
1724

1556

1198

1066

1245

1357

1198

1157

1343

1241

1288

1463
2100

2380

1680

1560

1650

1660

1730

1560

1920

1660

1790

1840

MOIP 5.0 
DOG

NUMBER

COMMENTS ON DEAD DOGS

F02P 2.0

MO3P 2.0

MO4P 2.0

FO5P 2.0

F06P 2.0

FO 7P 2.0

MO 8P 2.0

FO $9 P 2.0$

F $10 P 2.0$

M1 1P.2.0

M $12 P 2.0$

MO1P3.0

F02P.3.0

MO 3P 3.0 =

MO4P 3.0

FO5P 3.0

FO6P 3.0 :

FO 7P 3.0

MO 8 P 3.0

FO9P 3.0

F1OP.3.0

$M 11 P 3.0=$

M1293.0 :

$M 01 P 4.0$

FO2P 4.0 :

MO 3P 4. $0^{\circ}$

MO4P 4.0 :

FO $5 P 4.0$ :

F06P 4.0.

FO 7P 4.0

MO 8P 4.0.

FO 9P 4.0

F10P 4 .0

$M 11 P 4.0$

$M 12 P 4.0$
O STEOSARCOMA

- STEOSARCOMA

O STEOSARCOMA

O STEOSARCOMA

O STEOSARCOMA

SQUAMOUS CELL CAREI NOMA

A SP IRATION PNEUMONIA

O STEOSARCOMA

O STEOSARCOMA

OSTEOSARCONA

OSTEOSARCOMA

O STEOSARCOMA

O STEOSARCOMA

D STEOSARCOMA

O STEOSARCOMA

O STEOSARCOMA

O STEOSARCOMA

O STEOSARCOMA

O STEOSARCOMA

O STEOSARCOMA

O STEOSARCOMA

O STEOSARCOMA

O STEOSARCOMA

O STEOSARCOMA

O STEOSARCOMA

O STEOSARCOMA

O STEOSARCOMA

O STEOSARCOMA

O STEOSARCOMA

O STEOSARCOMA

O STEOSARCOMA

O STEOSARCOMA

O STEOSARCOMA

O STEOSARCOMA

O STEOSARCOMA 
DO G

NUMBER

F02P 5.0

MO 3P 5.0

MO 4P 5.0

F0 5P 5.0

F06P5.0

F07P 5.0

MO 8P 5.0

F0995.0
A T INJECTION AGE WEIGHT (DA YS) (KG)

I NJEC TED

$(\mu \mathrm{C} / \mathrm{KG})$. :

$8.75 \cdot 3.30$

150

515

566

691

407

482

497

552
8.10

3. 00

$9.18 \quad 3.17$

$8.77 \quad 2.77$

$7.90 \quad 2.57$

$8.33 \quad 2.99$

9.55

9.45

2.69

2.73
DATE

IN JECTED

D MO YR 30/9/65 DEATH

20353

10653

16.09 .53

$\begin{array}{llll}14 & 10 & 53\end{array}$

12.0554

$25 \quad 10 \quad 54$

$\begin{array}{llll}15 & 03 & 55\end{array}$

90955
DAYS SINCE

INJECI ION

1576

. 499

1562

2059

1194

1491

1192

1145
DOSE TO

SKELETON

( $R$ ADS)

7830

2340

7380

8690

4620

6630

4840

4750 
DOG

F02P5.0 M03P5.0 M0 4P 5.0 F0 5P.5.0 F06P 5.0 FO7P5.0 MO8P 5.0 FO9P 5.0
OSTEOSARCOMA + FRAC TUREED MANDIBLE LIVER DEGENERATION + ASCITES O STEOSARCOMA O STEOSARCOMA, LIVER DEGENERATI ON + HEPAT IC HEMORRHAGE O STEOSARCOMA OSTEOSARCOMA + CRIPPLING FRACTURE GINGIVITIS OSTEOSARCOMA, EPISTAXIS + CIRCULATORY COLLAPSE 
C. RADIUM-228 (MESOTHORIUM) *

\begin{tabular}{|c|c|c|c|c|c|c|c|c|}
\hline & $A T I N$ & JEC TIO & & & DATE & & DAYS & SINCE \\
\hline$D O G$ & $A G E$ & WEIGH T & I NJECTED & IN & $J E C$ & TED & INJEC & TION \\
\hline NUMBER & & ). $(K G)$. & $(\mu C / K G)$ & D & MO & $Y R$ & $30 / 9 / 65$ & DEA \\
\hline $01 M 0.0$ & 732 & 7.33 & & 4 & 1 & 54 & & 3451 \\
\hline & 545 & 6.94 & & 29 & 11 & 54 & 3958 & \\
\hline MO3MO.O & 579 & 13.0 & & 13 & 03 & 56 & 3488 & \\
\hline & 601 & 1.0 .3 & & 15 & 01 & 57 & 3180 & \\
\hline FOSMO:O & 671 & 11.2 & & 5 & 03 & 57 & 3131 & \\
\hline 0.0 & 492 & 7.56 & & 23 & 04 & 57 & 3082 & \\
\hline $07 M 0.0$ & 395 & 8.71 & & 4 & 5 & 57. & & 1414 \\
\hline FOTMO.OA & .594 & 10.9 & & 15 & 1 & 53 & 989 & \\
\hline O 8M0.0 & 654 & 11.6 & & 9 & 03 & 50 & 2031 & \\
\hline 0.0 & 575 & 12.4 & & 13 & 04 & 30 & 1996 & \\
\hline 0.0 & 581 & 13.3 & & 17 & 7 & 52 & 1171 & \\
\hline $1 M 0.0$ & 475 & 9.31 & & 18 & 9 & 52 & 1108 & \\
\hline$M 12 M 0.0$ & $6 \$ 5$ & 10.0 & & 22 & 12 & 50 & 1743 & \\
\hline
\end{tabular}

\begin{tabular}{|c|c|c|c|c|c|c|c|}
\hline FOIMO. 5 & 492 & 9.47 & .0173 & 17. & 7 & 52 & 1171 \\
\hline$F O 2 M 0.5$ & 492 & 9.15 & .0173 & 17 & 7 & 52 & 1171 \\
\hline MO3MO. 5 & 493 & 10.9 & .0199 & 18 & 9 & 52 & 110 \\
\hline MO4MO. 5 & 475 & 12.8 & .0199 & 18 & 9 & 62 & 116 \\
\hline F0 $5 M 0.5$ & 534 & 7.83 & .0172 & 23 & 10 & 52 & \\
\hline MOGMO. 5 & 510 & 10.3 & .0171 & 23 & 10 & 52 & 10.1 \\
\hline F07MO.5 & 492 & 8.87 & .0172 & 17 & 7 & 52 & 117 \\
\hline FOBMO. 5 & 654 & 12.6 & .0159 & 9 & 03 & 50 & 2 \\
\hline MOSMO.5 & 485 & 11.9 & .0170 & 13 & 04 & 50 & 19 \\
\hline MIOMO. 5 & 492 & 10.6 & .0174 & 17 & 7 & 62 & 11 \\
\hline F1LMO.5 & 50.5 & 7.82 & .0202 & 18 & 9 & 52 & \\
\hline M12MO.5 & 510 & 10.6 & .0165 & 23 & 10 & 62 & 107 \\
\hline
\end{tabular}

\begin{tabular}{|c|c|c|c|c|c|c|c|c|c|}
\hline FOIM1.0 & 718 & 7.75 & .0463 & 4 & 01 & 54 & & 2952 & 196 \\
\hline $\begin{array}{l}F 01 M 1.0 A \\
F 02 M 1.0\end{array}$ & 590 & 8.07 & .0512 & 23 & 10 & 52 & 107.3 & & \\
\hline MO3M1.0 & $\begin{array}{l}439 \\
575\end{array}$ & 8.25 & $\begin{array}{r}.0324 \\
0580\end{array}$ & 29 & 11 & 54 & 3958 & & \\
\hline MO4M1.O & 601 & 9.90 & .0481 & 15 & 01 & 57 & 3180 & הוצוב & \\
\hline F05M 1.0 & 658 & 8.80 & .0490 & 5 & 03 & 57 & 3131 & & \\
\hline MO6M 1.O & 521 & 10.6 & .0468 & 23 & 04 & 57 & 3082 & & \\
\hline FOTM1.O & 534 & 9.89 & .0489 & 4 & 6 & 57 & & 2159 & \\
\hline FOBM 1.0 & 654 & 12.4 & .0491 & 9 & 03 & 50 & 2031 & & \\
\hline MO9M 1.0 & 485 & 10.1 & .0504 & 13 & 04 & 50 & 1996 & & \\
\hline$M 1 O M 1.0$ & 492 & 9.43 & .0501 & 17 & 7 & 52 & 1171 & & \\
\hline$F 11 M: 1.0$ & 505 & 8.91 & .0613 & 18 & 9 & 52 & 1108 & & \\
\hline
\end{tabular}


DOG

NUMBER

FO $1 M 0.0$

FO2MO.O

MO $3 M O . O$

MO4MO.O

FO5MO.O

MOSMO.O

FO TMO.O

FOTMO.OA

FO BM O.O

MO9MO.O

M1OMO.O

F11M0.0

$M 12 M O . O$

FOIMO. 5

FO2MO. 5

MO $3 M O .5$

MO 4MO. 5

F05MO. 5

MO $6 M 0.5$

FOTMO. 5

F0 8MO. 5

MO9MO. 5

MIOMO. 5

FIIMO. 5

MI2MO. 5

FOIM1.0

FO1M1.OA

FO $2 M 1.0$

$M O 3 M 1.0$

MO4M1.O

F05M 1.0

MO6M 1.0

FOTM 1.0

FOSM 1.0

MO 9M 1.0

M1OM1.0

FIIMI.0
COMMENTS ON DEAD DOGS

PUR ULENT MENI NGOENCEPHAL ITIS

SIA TUS EPILEPTICUS

\section{HEMANG I OSARCOMA}

O STEOSARCOMA

HEMANG IOSARCOMA 


\begin{tabular}{|c|c|c|c|}
\hline \multirow{3}{*}{$\begin{array}{l}\text { DOG } \\
\text { NUMBER }\end{array}$} & \multicolumn{2}{|c|}{ AT INJECTION } & \multirow{3}{*}{$\begin{array}{r}\text { IN JEC } \\
(\mu \mathrm{C} /\end{array}$} \\
\hline & $A G E$ & WE I GHT & \\
\hline & I DAYS & $(K G)$ & \\
\hline$M 12 M 1.0$ & 528 & 9.27 & .049 \\
\hline FO $1 M 1.7$ & 510 & 7.52 & .151 \\
\hline FO 2M.1.7 & 560 & 9.90 & .183 \\
\hline MO3M1.7 & 576 & 11.0 & .180 \\
\hline MO4M 1.7 & 601 & 8.94 & .143 \\
\hline F05M 1.7 & 658 & 12.8 & .141 \\
\hline MO6M 1.7 & 521 & 10.0 & .144 \\
\hline FO TM 1.7 & 534 & 10.2 & .146 \\
\hline FC 8M 1.7 & $t 54$ & 10.8 & .148 \\
\hline MO9M 1.7 & 485 & 12.6 & .149 \\
\hline M1OM 1.7 & 492 & 10.1 & .124 \\
\hline$F 11 . M 1.7$ & 505 & 10.7 & .179 \\
\hline M12M1.7. & 524 & 9.28 & .153 \\
\hline
\end{tabular}

DATE DAYS SINCE DOSE TO INJECTED INJECTION SKELETON D MD YR 30/9/65 DEATH (RADS)

$23 \cdot 10 \quad 52 \quad 1073$

$\begin{array}{cccc}\text { FO M 2.0 } & 676 & 7.60 & .276 \\ \text { FO2M2.0 } & 517 & 8.25 & .194 \\ \text { MO3M2.0 } & 576 & 11.0 & .358 \\ \text { MO4M2.0 } & 601 & 9.88 & .282 \\ \text { F05M2.0 } & 509 & 8.30 & .295 \\ \text { MOGM2.0 } & 502 & 12.4 & .306 \\ \text { FO M 2.0 } & 534 & 10.1 & .298 \\ \text { FO M2.0 } & 654 & 12.4 & .300 \\ \text { MO9M2.0 } & 630 & 9.99 & .302 \\ \text { MIOM2.0 } & 430 & 11.2 & .311 \\ \text { F11M2.0 } & 505 & 7.03 & .381 \\ \text { M12M2.0 } & 524 & 9.47 & .306\end{array}$

$\begin{array}{rrrrr}4 & 01 & 54 & & 1780 \\ 29 & 11 & 54 & & 965 \\ 13 & 03 & 56 & & 619 \\ 15 & 1 & 57 & & 2282 \\ 5 & 3 & 57 & & 2688 \\ 23 & 4 & 57 & & 2674 \\ 4 & 6 & 57 & & 2239 \\ 9 & 03 & 50 & 2031 & \\ 13 & 4 & 50 & & 1254 \\ 17 & 7 & 52 & 1171 & \\ 18 & 9 & 52 & 1108 & \\ 23 & 10 & 52 & 1073 & \end{array}$

$\begin{array}{rrrrr}23 & 10 & 52 & 1073 & \\ 13 & 03 & 56 & & 2383 \\ 13 & 3 & 56 & & 2709 \\ 15 & .01 & 57 & & 2864 \\ 5 & 03 & 57 & 3131 & \\ 23 & 04 & 57 & 3082 & \\ 4 & 6 & 57 & & 2646 \\ 9 & 03 & 50 & 2031 & \\ 13 & 04 & 50 & 1996 & \\ 17 & 7 & 52 & 1171 & \\ 18 & 9 & 52 & 1108 & \\ 23 & 10 & 52 & 1073 & \end{array}$

733

723

424

600

2282

2674

2239

870

$65 \quad 198$

$19 \quad 355$

033

928

1382

1064

561

$\begin{array}{cccc}\text { FO 1M 3.0 } & 519 & 10.4 & .858 \\ \text { FO 2M 3.0 } & 460 & 6.70 & .612 \\ \text { MO 3M 3.0 } & 579 & 10.4 & .965 \\ \text { MO4M3.0 } & 601 & 10.2 & .916 \\ \text { FO SM 3.0 } & 531 & 8.51 & .940 \\ \text { MOSM 3.0 } & 502 & 9.09 & .953 \\ \text { FO TM 3.0 } & 534 & 9.94 & .907 \\ \text { FOBM 3.0 } & 633 & 11.8 & .950 \\ \text { MO M 3.0 } & 630 & 9.83 & .918\end{array}$

$\begin{array}{rrr}4 & 01 & 54 \\ 29 & 11 & 54 \\ 13 & 3 & 56 \\ 15 & 01 & 57 \\ 5 & 03 & 57 \\ 23 & 04 & 57 \\ 4 & 5 & 57 \\ 9 & 3 & 50 \\ 13 & 4 & 50\end{array}$

918

1833

1856

2075

$1185 \quad 2464$

$1176 \quad 1592$

$1869 \quad 2148$

$1421 ; 1906$

$1463 \quad 3145$

$1447 \quad 2158$

$1570 \quad 2277$ 
DOG

NUMBER

$M 12 M 1.0$

FO 1M1.7

FO 2M1.7

MO 3M 1.7

OSTEOSARCOMA

MO 4M 1.7

O STEOSARCOMA

FO5M 1.7

MO6M1.7

F07M 1.7

FO 8M1.7

MOSM 1.7

M1OPi 1.7

F11M1.7

M12M1.7

FO 1M2.0

D STEOSARCOMA

$\mathrm{FO} 2 \mathrm{M} 2.0$

CARCINOMA SMALL INTESTINE

$M O 3 M 2.0$

$M 04 M 2.0$

FO5M2.0

$M 06 M 2.0$

OSTEOSARCOMA

FOTM 2.0

FO $8 M 2.0$

MO $9 M 2.0$

M1OM 2.0

F11M2.0

$M 12 M 2.0$

F0.1M3.0 INTE STINAL: HE MORRHAGE PNE UMONIA

O STEOSARCOMA

OSTEOSARCOMA

OSTEOSARCOMA

CHR CNIC PANCREATITIS

R.ETICULUM CELL SARCOMA: OF VERTEBRA

FO 2M 3. 0

$M O 3 M 3.0$

$M 04 M 3.0$

FO $5 M 3.0$

MOGM3.0

F07M3.0

FO 8M3.0

MOSM3.0
OSTEOSARCOMA
O STEOSARCOMA
- STEOSARCOMA
O STEOSARCOMA
O STEUSARCOMA
- STEOSARCOMA
O STEOSARCOMA
O STEOSARCOMA
O STEOSARCOMA 


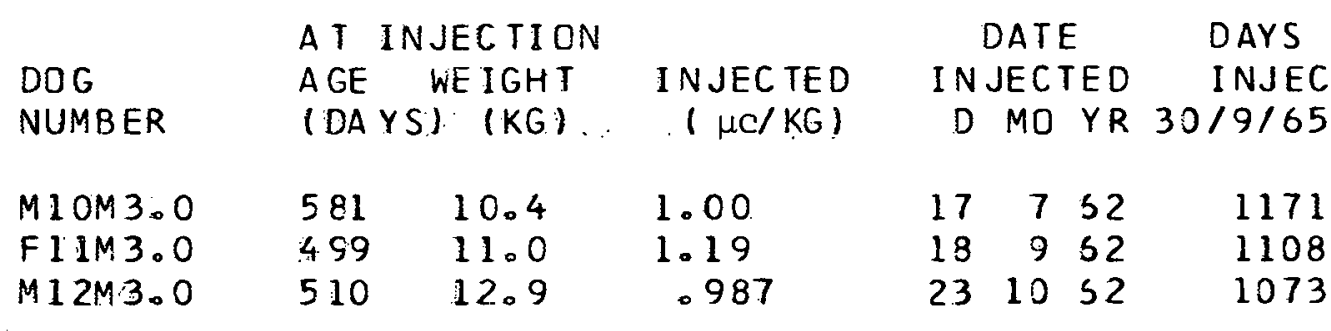

\begin{tabular}{|c|c|c|c|c|c|c|c|c|}
\hline FO1M4.0 & 510 & 7.56 & 2.60 & & .01 & 54 & 841 & 561 \\
\hline FO2M4.0 & 460 & 6.95 & 1.86 & 29 & 11. & 54 & 778 & \\
\hline MO3M4.0 & 579 & 9.65 & 3.37 & 13 & 03 & 56 & 418 & \\
\hline MO3M4.0A & 494 & 7.34 & 2.64 & 4 & $: 06$ & 57 & 1063 & \\
\hline MO4M4.O & 609 & $7: 84$ & 2.47 & 15 & 1 & 57. & 896 & 268 \\
\hline FOSM4.0 & 509 & 9.63 & 2.67 & 5 & 03 & 57 & 1064 & 43 \\
\hline MO6M 4.0 & 502 & 9.49 & 2.66 & 23 & 04 & .27 & 1121 & \\
\hline Fo7M4.0 & 544 & 8.40 & 2.67 & 4 & 05 & 57 & 1253 & \\
\hline FOIM5.0 & 494 & 7.77 & 8.11 & 4 & 01 & 54 & 232 & \\
\hline F02M5.0 & 460 & 7.35 & 5.46 & 29 & 11 & 54 & 780 & \\
\hline MO3M5.O & 579 & 8.87 & 10.4 & 13 & 3 & 56 & 688 & 141 \\
\hline MO4M5.O & 482 & 7.29 & 7.89 & 15 & 01 & 57 & 561 & \\
\hline F05M 5.0 & 658 & 11.1 & 8.48 & 5 & 3 & 57 & 770 & \\
\hline MO $6 M 5.0$ & 580 & 7.53 & 8.67 & 23 & 4 & 5.7 & 792 & 67 \\
\hline FO7M5.0 & 494 & 7.35 & 8.92 & 4 & .05 & 57 & 966 & 180 \\
\hline
\end{tabular}

${ }^{*}\left(\mu \mathrm{C} \mathrm{Th}^{228} / \mu \mathrm{C} \mathrm{Ra}{ }^{228}\right)$. injeçted $=0.15$ for FlMI.0, 2.0, 3..0, 4.0, 5.90.

$=0.03$ for F2MI.0, 1.7, 2.0, 3.0, 4.0, 5.0, M3Ml.0, $1.7,2.0,3.0,4.0,5.0$.

$=0.006$ for groups $4,5,6,7,8,9,10,11$, 12, and dogs F1M0.5, F2M0.5, M3MO.5, FIMIA, FIMI.7 and M3M4.0A. 
$D O G$

NUMBER

$M 10 M 3.0$

FIIM3.0

$M 12 M 3.0$
COMMENTS ON DEAD DOGS
FO1M4.O $\mathrm{FO} 2 \mathrm{M} 4.0$ $M 03 M 4.0$ $M 03 M 4.0 A$ $M 04 M 4.0$ F05M 4.0 $M 06 M 4.0$ FO $7 M 4.0$

FO1M5.0 F02M5.0 MO3M5.0 MO4M5.0 F05M5.0 M.O6M5.O F07M5.0
OSTEDSARCOMA + CRIPPLING FRACTURE

OSTEOSARCOMA

SSTR ANGULA TED INGUINAL HERNI A

O STEOSARCOMA, NEPHRITIS, ULCERATIVE GINGIVITIS + PNEUMONIA FRACTURED MANDIBLE + ULCERATIVE GINGIVITIS

OSTEOSARCOMA

OSTEOSARCOMA

D STEOSARCOMA

NEPHRITIS +. SEVERE ANEMI A

CRIPPLING FRACTURES

ULCERATIVE GINGIVITIS

CRIPPLING FRACTURE

ULCERATIVE GINGIVITIS

OSTEOSARCOMA: +CRI PPLING FRACTURE

LILERATIVE GINGIVITIS, MYOCARDIAL INFARCTION + GLAUCOMA 
D. THORIUM-228 (RADIOTHORIUM) \#

DOG

NUMBER

\begin{tabular}{|c|c|c|}
\hline MOLITO.O & 493 & 8.24 \\
\hline MO2TO.O & 488 & 7.28 \\
\hline F03T0.0 & 797 & 11.6 \\
\hline M04T0.0 & 591 & 8.10 \\
\hline MOSTO.O & 458 & 10.4 \\
\hline FO6T0.0 & 489 & 9.64 \\
\hline FO6TO.OA & 688 & 8.61 \\
\hline MOTTO.O & 517 & 10.5 \\
\hline MO8TO.O & 533 & 10.8 \\
\hline F09T0.0 & 569 & 8.28 \\
\hline F10T0.0 & 536 & 10.4 \\
\hline F $11 T 0.0$ & 530 & 9.45 \\
\hline$F 1210.0$ & 492 & 9.0 \\
\hline
\end{tabular}

AT INJECTION AGE WEIGHT (DAYS) (KG)

IN JEC TED $(\mu \mathrm{C} / \mathrm{K} G)$

\begin{tabular}{|c|c|c|c|c|}
\hline & Ar & & DAYS & INCE \\
\hline & $\mathrm{EEC}$ & TED & INJEC] & IION \\
\hline D & MO & $Y R$ & $30 / 9 / 65$ & DEATH \\
\hline 8 & 02 & 54 & 4252 & \\
\hline 28 & 09 & 54 & 4020 & \\
\hline 6 & 06 & 55 & & 2592 \\
\hline 18 & 10 & 55 & & 3072 \\
\hline 14 & 10 & 58 & 2543 & \\
\hline 10 & 1 & 61 & & 171 \\
\hline 15 & 12 & 30 & 1750 & \\
\hline 7 & 2 & 51 & & 1412 \\
\hline 24 & 5 & 51 & 1.590 & \\
\hline 29 & 6 & 5.1 & 1554 & \\
\hline 28 & 7 & 51 & 1525 & \\
\hline 4 & 5 & 53 & 849 & \\
\hline 9 & & 53 & 814 & \\
\hline
\end{tabular}

1283

1283

1283

1283

2.7. $03 \quad 52$

9.0230

4.: $6 \quad 53$

$10 \cdots 1: 81$

$7 \cdot 2 \cdot 51$

$24 \quad 5 \quad 51$

$29 \quad 6 \quad 31$

$28 \quad 7 \quad 51$

$4: 5 \quad 53$

9753

849

889

10

1724

1696

1590

1554

1525

849

814
DOSE TO

SKELETON

(RADS)

\section{M08T0. 2}

F09T0.2

FIOT0.2

F11T0.2

F12T0.2

530

.492

9.76
7.37

.00170

.00171

.00190 .

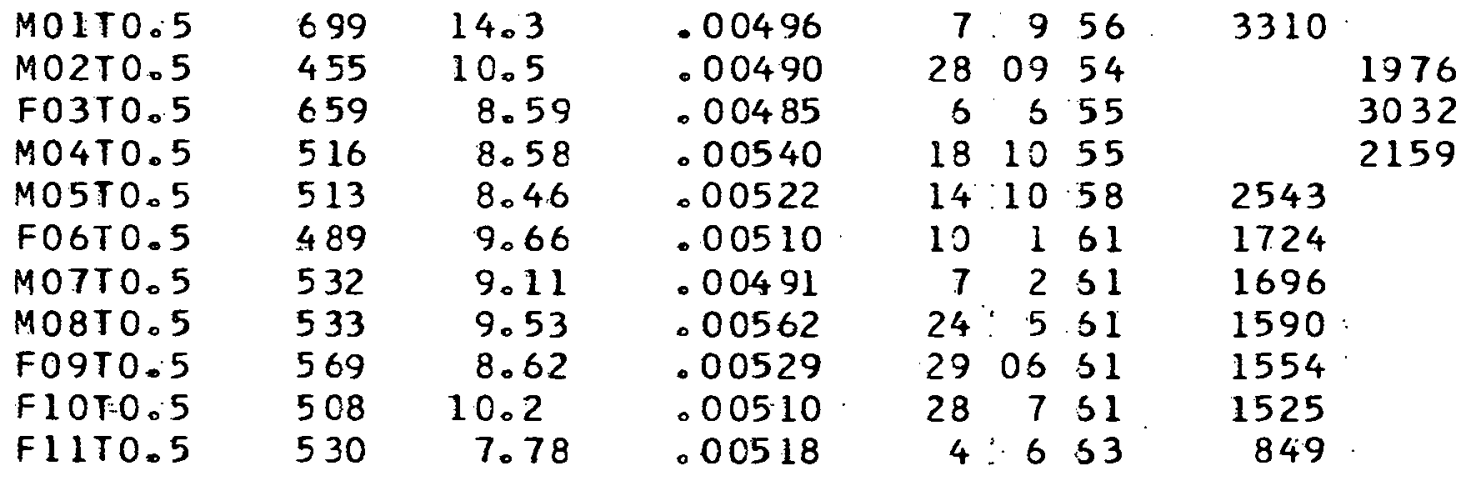

41

44

46 
DOG

NUMBER

COMMENTS ON DEAD.DOGS

MOLTO.O

MO2T0.O

F03T.0.0

M04TO.O

MO5TO.O

F06T0.0

F06T0.0A

MO7TO.O

MO8TO.O

FO9TO.0

FIOTO.0

F11T0.0

F12T0.0

BRA IN HEMORRHAGE

LYM PHOSARCOMA

TRA UMA

BRA IN HEMORRHAGE

MOLTO. 2

MO2TO.2

F03TO. 2

M04TO.2

MO5TO.2

M05TO.2A

STRANGULATION ON VOMITUS + GRAND MAL

F06T0.2

M07ro. 2

MO8TO.2

FO9TO. 2

F10TO.2

F11T0.2

F12T0.2

MO1T0.5

MO2T0.5

F03T0.5

MO4TO. 5

M05TO.5

F06T0.5

M07TO. 5

MO8T0.5

F09T0.5

F10T0.5

F11T0.5

STR ANGULATION ON VOMI.TUS + GRAND MAL

P YOMETRITIS + SECONDARY PERITONITIS

STATUS EPILEPTICUS + PNEUMONIA 
DOG

NUMBER

F12T0.5
AT INJECTION AGE WEIGHT (DAYS) (KG)

$$
9.94
$$

$\begin{array}{ll}9.36 & .0146 \\ 9.27 & .0146 \\ 8.84 & .0145 \\ 8.27 & .0146 \\ 1.9 & .0146 \\ 8.81 & .0150 \\ 9.18 & .0147 \\ 8.69 & .0166 \\ 10.0 & .0160 \\ 0.2 & .0150 \\ 7.55 & .0154 \\ 9.96 & .0167\end{array}$

$\begin{array}{ll}\text { MOIT1.0 } & 493 \\ \text { MO2T1.0 } & 699 \\ \text { FO3T1.0 } & 723 \\ \text { MO4T1.0 } & 699 \\ \text { MO5T1.0 } & 513 \\ \text { FO6T1.0 } & 489 \\ \text { MOTT1.0 } & 532 \\ \text { MO8T1.0 } & 533 \\ \text { FO9T1.0 } & 527 \\ \text { F1OT1.0 } & 508 \\ \text { F11T1.0 } & 521 \\ \text { F12T1.0 } & 472\end{array}$

493
699

723

699

489

532

5.33

527

508

472$$
.00567
$$

I NJEC TED

$(\mu \mathrm{C} / \mathrm{KG})$

.00567
DATE I N JECTED D MD YR

$9 \quad 753$

$\begin{array}{rrr}8 & 02 & 54 \\ 7 & 09 & 56 \\ 7 & 09 & 56 \\ 7 & 09 & 56 \\ 14 & 10 & 58 \\ 10 & 1 & 51 \\ 7 & 2 & 51 \\ 24 & 5 & 51 \\ 29 & 6 & 51 \\ 28 & 7 & 51 \\ 4 & 6 & 53 \\ 9 & 7 & 53\end{array}$

$\begin{array}{rrr}7.956 \\ 29 & 0954\end{array}$

60655

$\begin{array}{lll}18 & 10 & 55\end{array}$

9. 250

10. 151

$\begin{array}{lll}7 & 5 & 51\end{array}$

4. 553

24.551

$29 \quad 5 \quad 51$

$\begin{array}{lll}28 & 7 & 61\end{array}$

$\begin{array}{lll}4 & 5 & 53\end{array}$

9763

.0296

.0329

F12T1.5 465

$\begin{array}{lccl}\text { MOIT2.0 } & 491 & 10.2 & .0976 \\ \text { MO2T2.0 } & 483 & 9.16 & .0875 \\ \text { F03T2.0 } & 474 & 7.87 & .0908 \\ \text { MO4T2.0 } & 553 & 13.0 & .0900 \\ \text { MO4T2.0A } & 650 & 10.6 & .0899 \\ \text { M05T2.0 } & 598 & 9.112 & .0848 \\ \text { F06T2.0 } & 451 & 8.65 & .0879 \\ \text { M07T2.0 } & 5.17 & 8.85 & .0881\end{array}$

$\begin{array}{rrr}8 & 02 & 54 \\ 28 & 09 & 54 \\ 6 & 06 & 55 \\ 18 & 10 & 55 \\ 7 & 09 & 56 \\ 9 & 02 & 50 \\ 10 & 1 & 51 \\ 7 & 2 & 51\end{array}$

DAYS SINCE

INJECT ION

DEATH

814
DOSE TO SKELETON

(RADS)

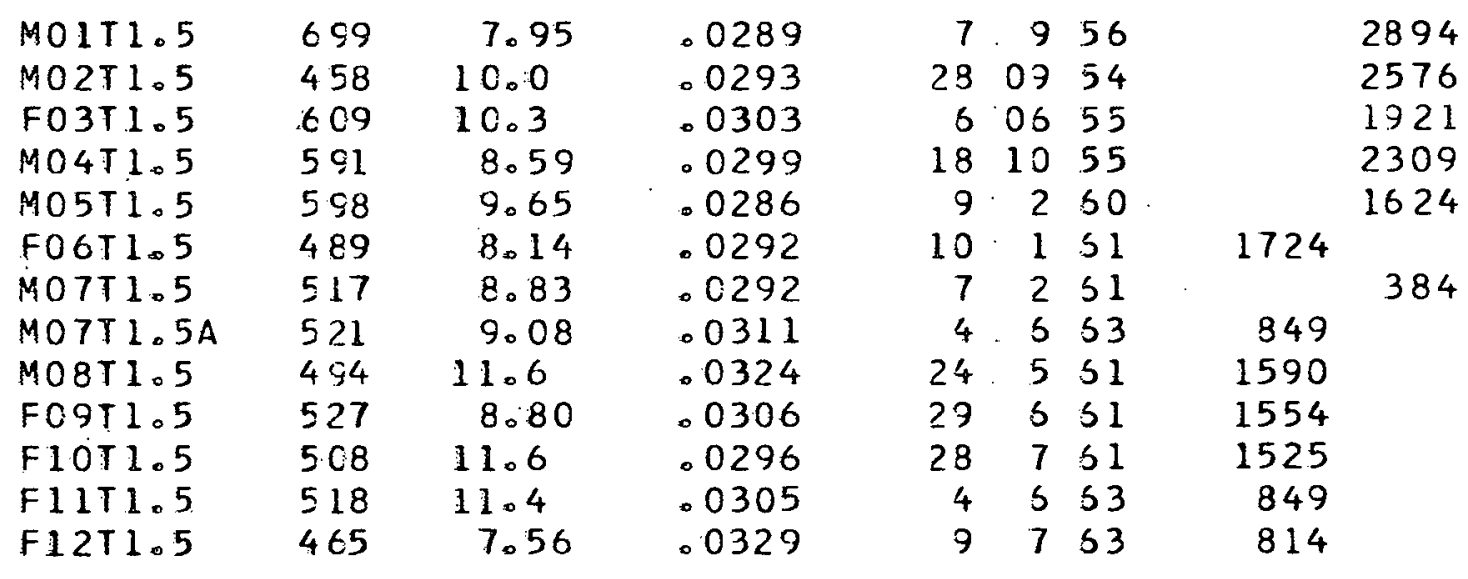

1282

693

1234

1541

78

1222

1085

1108

1015

258

257

249

257

223

95

611

695

70

625

556

583

558 
DOG

NUMBER

COMMENTS ON DEAD DOGS

F12T0.5 5

MOITI.O

MO2T1.0

F03T1.0

M04T1.0

MO5T1.0

FO6T1.0

MOTT1.0

MOBTLOO

FO9TL.0

F1OT 1.0

F11T1.0

F $12 T 1.0$
OSTEOSARCOMA

OSTEUSARCOMA THYROID CARCINOMA PERIANAL ADENOCARCINOMA
MO1T1.5

MO2T1.5

F03T1.5

MO4T1.5

M05T1.5

F06T1.5

MO7T 1. 5

MO7T1.5A

MO8T1.5

FO9T 1.5

F10T1.5

F11T1.5

F12T1.5

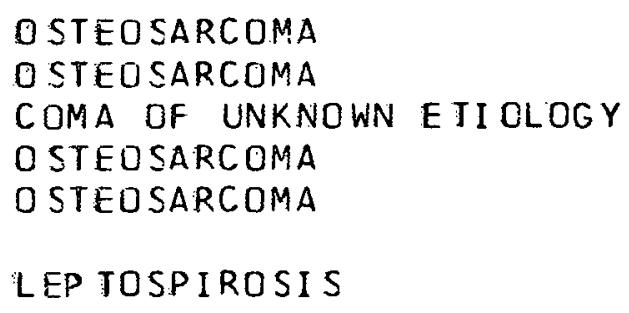

MOTT2.0 OSTEOSARCOMA 


\begin{tabular}{|c|c|c|c|}
\hline $\begin{array}{l}\text { DOG } \\
\text { NUMBER }\end{array}$ & $\begin{array}{l}\text { A T } \\
\text { AGE } \\
\text { I DA }\end{array}$ & $\begin{array}{l}\text { JEC I I ON } \\
\text { WE I GHT } \\
)(K G)\end{array}$ & $\begin{array}{r}\text { IN JEC } \\
(\mu \mathrm{C})\end{array}$ \\
\hline $\begin{array}{l}\text { MO8T2.0 } \\
\text { F09T2.0 } \\
\text { F1OT2.0 } \\
\text { FI 1T2.0 } \\
F 12 T 2.0\end{array}$ & $\begin{array}{l}533 \\
527 \\
508 \\
518 \\
464\end{array}$ & $\begin{array}{c}10.7 \\
8.09 \\
10.7 \\
10.8 \\
8.92\end{array}$ & $\begin{array}{l}.098 \\
.097 \\
.091 \\
.090 \\
.100\end{array}$ \\
\hline $\begin{array}{l}\text { MO1T3.0 } \\
\text { MO2T3.0 } \\
F 03 T 3.0 \\
\text { MO4T3.0 } \\
\text { MO5T3.0 } \\
\text { F06T3.0 } \\
\text { MOTT3.0 } \\
\text { MO8T3.0 } \\
F 09 T 3.0 \\
F 10 T 3.0 \\
F 11 T 3.0 \\
F 12 T 3.0\end{array}$ & $\begin{array}{l}314 \\
458 \\
471 \\
606 \\
571 \\
451 \\
427 \\
494 \\
511 \\
508 \\
518 \\
459\end{array}$ & $\begin{array}{c}9.15 \\
11.9 \\
12.0 \\
9.69 \\
10.7 \\
8.83 \\
9.90 \\
10.1 \\
11.5 \\
9.26 \\
10.3 \\
11.5\end{array}$ & $\begin{array}{l}.301 \\
.301 \\
.272 \\
.285 \\
.269 \\
.282 \\
.266 \\
.313 \\
.298 \\
.280 \\
.290 \\
.320\end{array}$ \\
\hline
\end{tabular}

DATE DAYS SINCE I N JECTED INJECTION

D MO YR $30 / 9 / 65$ DEATH

$24 \quad 5.51$

$29 \quad 6 \quad 51$

28751

4. 653

9753

849

814
1078

1209

1022
DOSE TO

SKELETON

(RADS)

641

677

584 $\begin{array}{rrr}8 & 02 & 54 \\ 28 & 09 & 54 \\ 6 & 06 & 55 \\ 18 & 10 & 55 \\ 9 & 02 & 50 \\ 10 & 1 & 51 \\ 7 & 2 & 51 \\ 24 & 5 & 51 \\ 29 & 6 & 51 \\ 28 & 7 & 51 \\ 4 & 5 & 53 \\ 9 & 7 & 53\end{array}$

$\begin{array}{llll}8 & 02 & 54\end{array}$

28.0954

$6.06 \quad 55$

181055

$\begin{array}{llll}\text { MO1T4.0 } & 480 & 8.32 & .882 \\ \text { M02T4.0 } & 458 & 8.32 & .916 \\ \text { F03T4.0 } & 461 & 7.25 & .800 \\ \text { M04T4.0 } & 6 C 6 & 8.81 & .835\end{array}$

645

833

763

793

1879

859

1736

547

1164

$801 \quad 1575$

890 . 1584

1156

1909

861

1536

685

1566

1062

1933

971

1732

791

1591

$804 \quad .1773$

M04T4.0 606

$8.81 \quad .835$

793

4237

5185

4290

4587

$\begin{array}{llll}M 01 T 5.0 & 480 & 9.48 & 2.76 \\ M 02 T 5.0 & 483 & 8.22 & 2.63\end{array}$

$802: 54$

$28.09 \quad 54$

212

5457

97

2535 
$D O G$

NUMBER

COMMENTS ON DEAD DOGS

MO8T2.0

OSTEOSARCOMA

F09T2.0

O STEOSARCOMA

F10 2.0

D STEOSARCOMA

F11T2.0

F12T2.0

MOLT3.O

O STEOSARCOMA + SEVERE ANEMI A

$M 02 T 3.0$

OSTEOSARCOMA + TRAUMA

F03T3.0

O STEOSARCOMA

MO4T3.0

O STEOSARCOMA

MO5T 3.0

O STEOSARCOMA

FO $6 \mathrm{~T} 3.0$

O STEOSARCOMA

MOTT3.0

O STEOSARCOMA

MO8T3.0

D STEOSARCOMA

F09T3.0

- STEOSARCOMA

F10T3.0

O STEOSARCOMA

F11T3.0

O STEOSARCOMA

F12T3.0

HEMANGIOSARCOMA

MO1T4.0

OSTEOSARCOMA + CRIPPLING FRACTURE

M02T4.O

OSTEOSARCOMA OCRIPPLING FRACTURE * NEPHRIIIS

F03T4.0

WLCERATIVE. GINGIVITIS + NEPHRITIS

MO4T 4.0

ULCERATIVE GINGIVITIS.

MO1T5.0 NEPHRITIS

MO2T5.0 PANCYTOPENIA, 
E. STRONTIUM-90

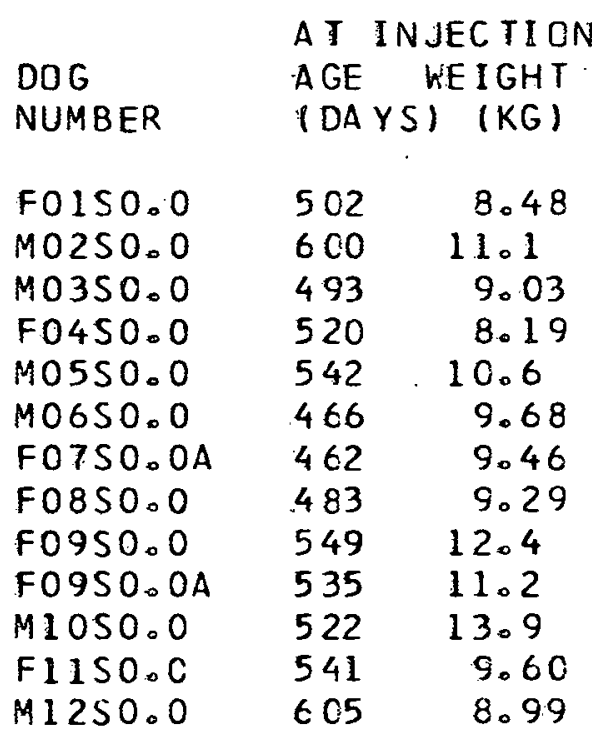

DATE DAYS SINCE

I N JEC TED

( $\mu \mathrm{C} / K G$ )

INJECTED

D MO YR $30 / 9 / 65$ DEATH

DOSE TO

SKELETON

(RADS)

$\begin{array}{rrrr}18 & 01 & 55 & 3908 \\ 14 & 02 & 56 & 3516 \\ 11 & 09 & 57 & 2941 \\ 15 & 10 & 57 & 2907 \\ 19 & 11 & 57 & 2872 \\ 27 & 05 & 58 & 2683 \\ 7 & 01 & 59 & 2458 \\ 19 & 05 & 59 & 2326 \\ 11 & 08 & 59 & \\ 4 & 5 & 53 & 849 \\ 29 & 09 & 59 & 2193 \\ 3 & 11 & 59 & 2158 \\ 6 & 01 & 50 & 2094\end{array}$

708
$1801 \quad 55$

140256

$1402 \quad 56$

$\begin{array}{lll}11 & 09 & 57\end{array}$

$\begin{array}{lll}15 & 10 & 57\end{array}$

$\begin{array}{lll}19 & 11 & 57\end{array}$

$\begin{array}{lll}27 & 05 & 58\end{array}$

111158

190559

110859

290959

$311: 59$

5.0150

$\begin{array}{ll} & 308 \\ 3516 & \\ 3516 & \\ 2941 & \\ 2907 & \\ & 2705 \\ 2683 & \\ 2515 & \\ 2326 & \\ 2242 & \\ 2193 & \\ 2158 & \\ 2094 & \end{array}$

308

21

69

$1402 \quad 56 \quad 3516$

$14.0256 \quad 3516$

$110957 \quad 2941$

$\begin{array}{llll}15 & 10 & 57 & 2907\end{array}$

$\begin{array}{lll}19 & 11 & 57\end{array}$

5. 353

$\begin{array}{lll}27 & 05 & 58\end{array}$

11.1158

19559

$1103: 59$

290959

1715

161

939

2683

2515

2242

1973

215

2193

$10.2 \quad 1.60$

$\begin{array}{rr}8.47 & 2.03 \\ 10.0 & 1.62 \\ 13.6 & 1.66\end{array}$

13.6 
DOG

NUMBER

FOISO.O

MO2SO.O

M03SO.O

F04SO.O

M0550.O

MO6SO.O

FO750.0A

FO 850.0

F0950.0

FO9SO.OA

TRA UMA

MIOSO.0

F11S0.0

M12SO.O

F01S1.0

FOIS1.0A

MO2SL.O

M0351.O

F04S1.0

MO5S1.0

M06S1.0

F0751.0

FOBS1.0

FO9S1.0

M1OS1.0

F 1151.0

$M 1251.0$

F0151.7

MO2S1.7

M03S1.7

F04S1.7

M05S1.7

MO5S1.7A

M06S1.7

F07S1.7

F08S1.7

F0951.7

MIOS1.7
SACRIFICED -IMPROPER I NJEETION AGE-

STA TUS EPILEPTICUS

COMA OF UNKNOWN ETIOLOGY

IS IA IUS EPILEPTICUS, CHRONIC PANCREATITIS 
DOG
NUMBER
AT INJECTIION AGE WE IGHT (DAYS) (KG).
DATE

IN JEC TED

(. $\mu C / K G)$

F11S1.?

M12S1.7
$543 \quad 11.0$

$607 \quad 11.9$
1.68

1.68
IN JECTED

D MO YR

$\begin{array}{lll}3 & 11 & 59\end{array}$

50150
DAYS SINCE

INJECI ION

30/9/65 DEATH
DOSE TO

SKELETON

(RADS)

$\begin{array}{cccc}\text { FO1S2.0 } & 502 & 5.59 & 3.70 \\ \text { MO2S2.0 } & 567 & 8.97 & 3.42 \\ \text { MO3S2.0 } & 494 & 7.82 & 3.39 \\ \text { FO4S2.0 } & 522 & 9.68 & 3.41 \\ \text { MOSS2.0 } & 560 & 8.72 & 3.24 \\ \text { MO6S2.0 } & 466 & 9.19 & 3.50 \\ \text { FO TS2.0 } & 488 & 11.2 & 3.19 \\ \text { FO8S2.0 } & 465 & 9.49 & 4.14 \\ \text { FO9S2.0 } & 473 & 14.1 & 3.28 \\ \text { M1OS2.0 } & 508 & 10.7 & 3.34 \\ \text { F11S2.0 } & 543 & 10.4 & 3.41 \\ \text { M12S2.0 } & 607 & 11.6 & 3.49\end{array}$

18155

2158

2094

\begin{tabular}{|c|c|c|}
\hline $\begin{array}{l}F 01 S 3.0 \\
M O 2 S 3.0 \\
M O 3 S 3.0 \\
F O 4 S 3.0 \\
M O S S 3.0 \\
M O 6 S 3.0 \\
F O 7 S 3.0 \\
F O 8 S 3.0 \\
F O 9 S 3.0 \\
M 1 O S 3.0 \\
F 11 S 3.0 \\
M 12 S 3.0\end{array}$ & $\begin{array}{l}468 \\
565 \\
494 \\
527 \\
557 \\
466 \\
486 \\
465 \\
468 \\
519 \\
541 \\
605\end{array}$ & $\begin{array}{c}7.36 \\
9.62 \\
11.4 \\
9.17 \\
8.90 \\
9.44 \\
9.80 \\
12.5 \\
10.0 \\
12.5 \\
9.00 \\
8.43\end{array}$ \\
\hline
\end{tabular}

$\begin{array}{llll}18 & 0155 \quad 3908\end{array}$

$14.0256 \quad 3516$

$\begin{array}{llll}11 & 09 & 57 & 2941\end{array}$

$\begin{array}{lll}15 & 10.57 & 2907\end{array}$

$\begin{array}{llll}19 & 11 & 57 & 2872\end{array}$

$\begin{array}{llll}27 & 05 & 58 & 2683\end{array}$

$111158 \quad 2515$

$\begin{array}{llll}19 & 05 & 59 & 2326\end{array}$

$1108 \quad 59 \quad 2242$

$\begin{array}{llll}29 & 09 & 59 & 2193\end{array}$

$\begin{array}{llll}3 & 1159 & 2158\end{array}$

$\begin{array}{llll}5 & 0150 \quad 2094\end{array}$

$\begin{array}{cccc}\text { F01S4.0 } & 468 & 8.74 & 33.3 \\ \text { MO2S4.0 } & 567 & 11.2 & 32.6 \\ \text { M03S4.0 } & 593 & 9.83 & 32.1 \\ \text { F04S4.0 } & 528 & 8.24 & 32.1 \\ \text { MO5S4.0 } & 562 & 9.65 & 30.6 \\ \text { M06S4.0 } & 504 & 16.0 & 32.7 \\ \text { F07S4.0 } & 478 & 10.9 & 30.9 \\ \text { FO8S4.0 } & 465 & 10.9 & 40.6\end{array}$

$\begin{array}{lll}18 & 01 & 55\end{array}$

$14: 0256$

$\begin{array}{lll}11 & 09 & 57\end{array}$

$\begin{array}{lll}15 & 10 & 57\end{array}$

191157

3.0958

111158

190559
3269

480 
DOG

NUMBER

F11S1.7

M12S1.7

F01S2.0

$M 0252.0$

$M 0352.0$

F0 452.0

$M 0552.0$

$M 0652.0$

F0752.0

F0852.0

F0952.0

$M 10 S 2.0$

FIIS2.0

M12S2.0

FO1S3.0

MO2S3.0

MO3S 3.0

FO4S3.0

MO5S3.0

M06S3.0

F0753.0

F0.853.0

FOSS3.0

M1OS3.0

F1153.0

M12S3.0

F0154.0

M0254.0

MO 354.O

F04S4.0

M0554.0

M0654.0

F0754.0

F0854.0

COMMENTS ON DEAD DOGS

BAC TERIAL PNE UMONIA

A




\begin{tabular}{|c|c|c|c|c|c|c|c|c|c|}
\hline $\begin{array}{l}\text { DOG } \\
\text { NUMBER }\end{array}$ & $\begin{array}{l}A T I N \\
A G E \\
I D A Y S\end{array}$ & $\begin{array}{l}\text { JEC II ON } \\
\text { WE IGHT } \\
)(K G)\end{array}$ & $\begin{array}{c}\text { I N JEC TED } \\
(\mu \mathrm{C} / \mathrm{KG})\end{array}$ & $\begin{array}{r}\text { IN } \\
\text { D }\end{array}$ & $\begin{array}{l}\text { DATE } \\
\text { JECT } \\
\text { MD }\end{array}$ & $\begin{array}{l}\text { E } \\
\text { TED } \\
\text { YR }\end{array}$ & $\begin{array}{r}\text { DAYS } \\
\text { INJEC } \\
30 \% 9 / 65\end{array}$ & $\begin{array}{l}\text { S INCE } \\
\text { T ION } \\
\text { DEATH }\end{array}$ & $\begin{array}{l}\text { DOSE TO } \\
\text { SKELETO } \\
\text { (RADS) }\end{array}$ \\
\hline $\begin{array}{l}F 09 S 4.0 \\
M 10 S 4.0 \\
F 11 S 4.0 \\
M 12 S 4.0\end{array}$ & $\begin{array}{l}468 \\
517 \\
542 \\
605\end{array}$ & $\begin{array}{l}9.56 \\
8.20 \\
8.86 \\
10.9\end{array}$ & $\begin{array}{l}30.6 \\
31.3 \\
32.7 \\
32.3\end{array}$ & $\begin{array}{r}11 \\
29 \\
3 \\
6\end{array}$ & $\begin{array}{l}08 \\
09 \\
11 \\
01\end{array}$ & $\begin{array}{l}59 \\
59 \\
59 \\
30\end{array}$ & $\begin{array}{l}2242 \\
2193 \\
2094\end{array}$ & 2114 & 3324 \\
\hline 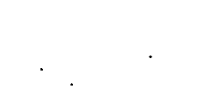 & & ; & & & & & & & \\
\hline $\begin{array}{l}\text { FO1S5.0 } \\
M 02 S 5.0 \\
M 02 S 5.0 A \\
M 03 S 5.0 \\
F 04 S 5.0 \\
M 05 S 5.0 \\
M 0655.0 \\
M 0655.0 A \\
F 0755.0 \\
F 0855.0 \\
F 09 S 5.0 \\
M 10 S 5.0 \\
F 11 S 5.0 \\
M 12 S 5.0\end{array}$ & $\begin{array}{l}434 \\
551 \\
545 \\
507 \\
528 \\
621 \\
504 \\
462 \\
478 \\
535 \\
459 \\
517 \\
542 \\
606\end{array}$ & $\begin{array}{l}9.38 \\
12.2 \\
11.4 \\
10.3 \\
11.4 \\
8.53 \\
9.33 \\
11.2 \\
10.2 \\
11.2 \\
8.82 \\
8.55 \\
8.97 \\
12.5\end{array}$ & $\begin{array}{c}103 . \\
102 . \\
96.6 \\
102 . \\
105 . \\
95.2 \\
98.8 \\
94.2 \\
92.7 \\
90.5 \\
93.5 \\
95.9 \\
102 . \\
99.2\end{array}$ & $\begin{array}{r}18 \\
14 \\
7 \\
15 \\
15 \\
19 \\
3 \\
7 \\
11 \\
7 \\
11 \\
29 \\
3 \\
6\end{array}$ & $\begin{array}{r}01 \\
02 \\
1 \\
10 \\
10 \\
11 \\
9 \\
01 \\
11 \\
01 \\
03 \\
09 \\
11 \\
01\end{array}$ & $\begin{array}{l}55 \\
56 \\
59 \\
57 \\
57 \\
57 \\
58 \\
59 \\
58 \\
59 \\
59 \\
59 \\
59 \\
50\end{array}$ & . & $\begin{array}{r}960 \\
255 \\
1740 \\
2256 \\
1448 \\
1285 \\
35 \\
1021 \\
1129 \\
1469 \\
1982 \\
990 \\
1667 \\
1165\end{array}$ & $\begin{array}{r}8127 \\
3202 \\
12007 \\
16297 \\
9281 \\
9930 \\
647 \\
11616 \\
10822 \\
10941 \\
13569 \\
7744 \\
9956 \\
8327\end{array}$ \\
\hline
\end{tabular}


DOG

F0954.0

$M 1054.0$

F1154.0

$M 1254.0$

F0155.0

$M 0255.0$

MO2S5.0A

MO3S5.0

F04S5.0

MO5S5.0

M0655.0

M0655.0A

F075 5.0

F08S5.0

FO9S5.0

M 1055.0

FI IS 5.0

M12S5.0
O STEUSARCOMA

$\subseteq$ TR ANGULATED INGUINAL HERNIA

O STEOSARCOMA

O STEOSARCOMA

O STEOSARCOMA

SEVERE ANEMIA, AUTOAGGLUTINATION, INFARCTION, SPLENOMEGALY INTESTINAL HEMORRHAGE

DSTEOSARCOMA, INFARCTION + THROMBOCYTOPENIA

STATUS EPILEPTICUS

OSTEOSARCOMA

SQUAMOUS CELL CARCINOMA ARISING FROM FRONTAL SINUS

SEVERE ANEMIA + THROMBOCYTOPENIA

HEMANGIOSARCOMA (LEFT MANDIBLE)

HEMANG IOSARCOMA (RIB) 
TABLE II.TEST ANI MALS (SEPT.30,1965)

A. RADIUM-226*

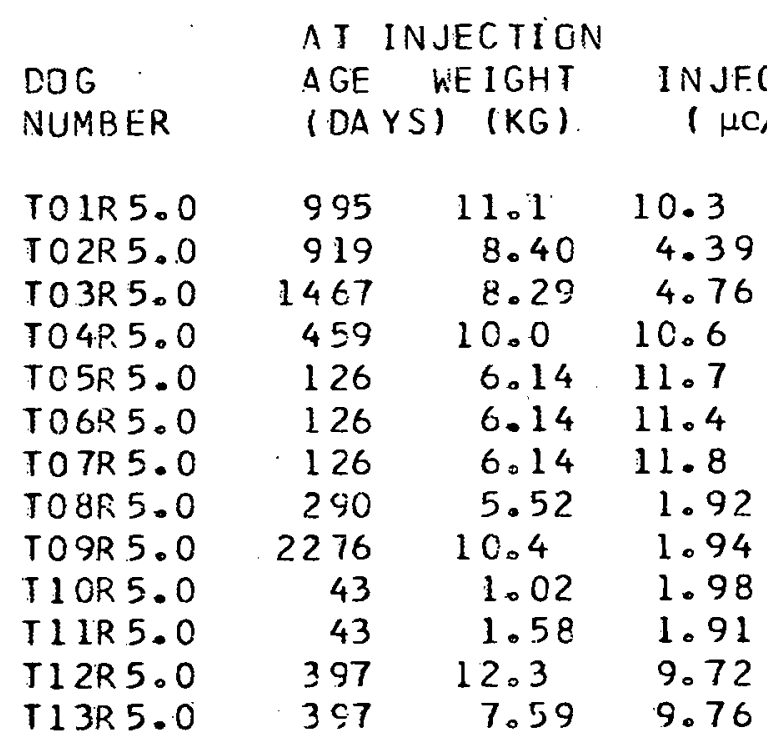

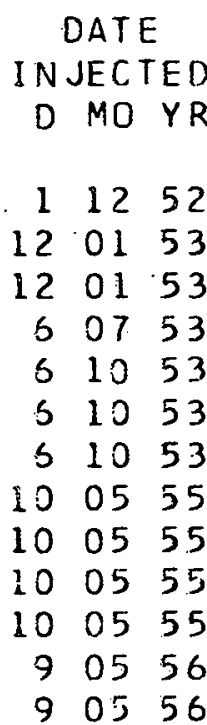

DAYS SINCE INJECTION $30 / 9 / 65$ DEATH

1074

1368

428

1

1

1

58

58

49

49

225

188
DOSE TO SKELETON (RADS)

$\begin{array}{llll}T 14 R 4.0 & 674 & 8.12 & 3.17 \\ T 15 R 4.0 & 672 & 9.03 & 3.11\end{array}$

110756

9469

4724

1316

24

25

24

25

488

585

293

363

2707

2319

110756

380

2127

5117

$\begin{array}{llll}\text { T16R5.0 } & 604 & 12.4 & 9.68 \\ \text { T17R5.OH } & 383 & 12.2 & 9.87 \\ \text { TI 8R5.CH } & 383 & 11.1 & 10.8 \\ \text { TI9R5.OH } & 383 & 11.3 & 10.7 \\ \text { T2OR.5.OH } & 383 & 11.4 & 10.6 \\ \text { T21P.5.OH } & 381 & 11.8 & 10.1 \\ \text { T22R5.OH } & 381 & 11.9 & 10.1\end{array}$

$\begin{array}{lll}11 & 07 & 57\end{array}$

$\begin{array}{lll}28 & 10 & 58\end{array}$

12

$28 \cdot 10 \quad 58$

$\begin{array}{lll}23 & 10 & .58\end{array}$

$\begin{array}{lll}28 & 10 & 58\end{array}$

$28 \quad 10 \quad 58$

$\begin{array}{ll}29 & 10 \quad 58\end{array}$

1147

183

1226

13655

1219

12628

1330

11580

326

12937

3710

587

5911

$\begin{array}{llcl}\text { T23R4.OH } & 384 & 9.50 & 4.05 \\ \text { T24R4.OH } & 384 & 11.9 & 3.24 \\ \text { T25R4.OH } & 379 & 11.3 & 3.42 \\ \text { T26R4.OH } & 379 & 11.0 & 3.48 \\ \text { T27R4.OH } & 372 & 11.5 & 3.34\end{array}$

$\begin{array}{lll}25 & 11 & 58\end{array}$

1471

4340

$\begin{array}{lll}25 & 11 & 58\end{array}$

1505

5593

$\begin{array}{llll}25 & 11 & 58\end{array}$

1309

4672

$\begin{array}{lll}25 & 11 & 58\end{array}$

1780

4719

$\begin{array}{lll}25 & 11 & 58\end{array}$

1414

3382

$\begin{array}{llll}\text { T28R 3.0H } & 372 & 11.7 & 1.11\end{array}$

$25 \quad 11 \quad 58$

387

357

$\begin{array}{llll}T 29 R 5.0 & 474 & 13.5 & 10.4\end{array}$

30359

216

3404 
DOG

TO1R5.0

TO2R 5.0 TO $3 R 5.0$ TO4R 5.0 TOSR 5.0 TOSR 5.0 TOTR 5.0 TORR 5.0 TO SR 5.0 TIOR 5.0 II IR 5.0 TI $2 R 5.0$ T1 $3 R 5.0$

$T 14 R 4.0$ $T 15 R 4.0$

T1 6R 5.0

TI TR5.OH TI.8R $5.0 \mathrm{OH}$ T19R 5.O.H T2OR 5.OH T21R5.OH T22R5.OH

T23R 4. OH $\mathrm{T} 24 \mathrm{R} 4 . \mathrm{OH}$ $\mathrm{T} 25 \mathrm{R} 4 . \mathrm{OH}$ T26R 4.OH T27P.4.OH

T28R.3.OH
OSTEUSARCOMA OSTEOSARCOMA SPECIAL STUDY SPECIAL STLDY SPECIAL STUDY SPECIAL STLDY SPECIAL STLDY SPECIAL STUDY SPECIAL SIUDY SPECIAL STUDY SPECIAL STUDY SPECIAL STUDY SPECIAL STUDY

SPECIAL STUDY O STEOSAPCOMA

SPECIAL STUDY

O STEOSARCOMA + ULCERATIVE GINGIVITIS

O STEUSARCOMA + ULCERATIVE GINGIVITIS

CSTEOSARCOMA + ULCERATIVE: GINGIVITIS

OSTEOSARCOMA + ULCERATIVE GINGIVITIS

NEPHRITIS

CRIPPLING FRACTURES

OSTEU SARCOMA

OSTEUSARCOMA

OSTEOSARCOMA

OSTEOSARCOMA

OSTEOSAR.COMA 
DOG

NUMBER

T3OR 5.0

T31R.5.0
AT INJECTION AGE WEIGH T (DAYS) (KG)
DATE INJEC TED $(\mu C / K G)$
IN JECTED

DAYS SINCE. INJECTION

D MO YR 30/9/65 DEATH

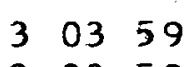

$\begin{array}{lll}3 & 03 & 59\end{array}$

$\begin{array}{lll}474 & 11.5 & 10.4\end{array}$

$\begin{array}{lll}471 & 10.5 & 10.4\end{array}$

471

471

11.4

1.13

T $332 R 3.0$

T $34 R 3.0$

T35R 3.0 J

10.6

1.15

470

15.7

1.12

9.44

.951

$\begin{array}{rrr}3 & 03 & 59 \\ 3 & 3 & 59 \\ 3 & 3 & 59 \\ 5 & 5 & 59\end{array}$

2294

1822

1737

178

303

$$
670
$$

DOSE TO SKELETON (RADS)

695

10.2

2.99

$T 36 R 4.0$
$T 37 R 4.0$

T $38 R 4.0$

695

9.53

3.00

$695 \quad 10.1$

3.02

22.1250.

1154

1627

1503

221250

$\begin{array}{lll}3 & 4 & 52 \\ 3 & 4 & 52\end{array}$

7
63

1

THIR.1.0

899

13.0

.0483

.0487

3452

$\begin{array}{lll}4 & 4 & 32 \\ 4 & 4 & 52\end{array}$

7
64

2
11

T43R 1.7

967

14.0

.146

.0145

$4 \quad 4: 52$

$\begin{array}{lll}5 & 4 & 32 \\ 5 & 4 & 52\end{array}$

.941
.928

68

7
69

71

12

$810 \quad 12.5$

103

T4 TR 6.0

T48R6.0
99

2842
5.27. 29.4

$11.2 \quad 25.1$ $\begin{array}{lll}11 & 5 & 62\end{array}$

$27 \quad 1232$
352

49 1597
T 4 9R:5.0

T 5 OR: 5.0

TSIR 5.0

T $52 R 5.0$
485

485

418

$418 \quad 10.7$
7.54

7.46

8.48

8.57
2:553

2. 5.53

3553

8563
.111

15

92

15 
DOG

NUMBER

COMMENTS ON DEAD DOGS

T3OR5.0 NEPHRITIS

T31R:5.0 NEPHRITIS

T32R3.0 OSTEOSARCOMA

T33R 3.0 OSTEOSARCOMA, NEPHRITIS

T34R3.0 OSTEOSARCOMA

T35R3.0J SPECIAL STUDY

T36R4.0 : OSTEOSARCOMA

T37R 4.0 OSTEOSARCOMA

T38R 4.0 OSTEOSARCOMA

T4OR 1.0 SPECIAL STUDY

T4IR 1.0 SPECIAL STUDY

T42R1.7 SPECIAL STUDY

T43R1.7 SPECIAL STUDY

$T 44 R 3.0$

SPECIAL STUDY

T45R3.0 SPECIAL STUDY

T46R3.0 SPECIAL STUDY

T47R6.0 SPECIAL STUDY

T48R6.0 LEUKOPENIA, PNEUMONIA + SPECIAL MELANOMA STUDY

T49R5.0 SPECIAL STUDY

TSOR 5.0 SPECIAL STUDY

T5IR 5.0 SPECIAL STUDY

TS2R5.0 SPECIAL STUDY 


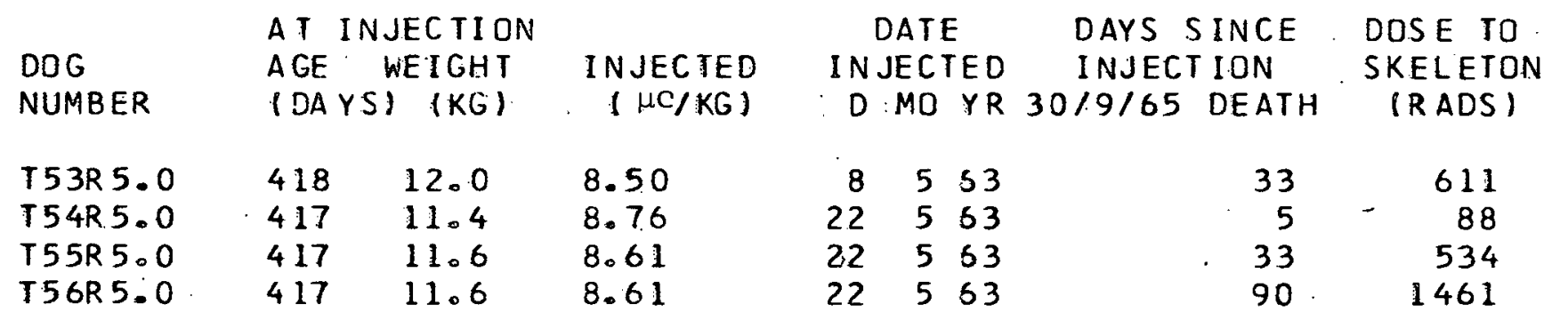

$\begin{array}{lccccccr}\text { T5TR 4.0 } & 501 & 12.1 & 2.72 & 15 & 963 & 14 & 54 \\ \text { T58R4.0 } & 496 & 11.7 & 2.41 & 15 & 853 & 61 & 286 \\ \text { T59R 4.0 } & 496 & 9.64 & 2.57 & 15 & 853 & 63 & 272 \\ \text { T6OR4.0 } & 490 & 12.1 & 2.33 & 15 & 853 & 117 & 415 \\ \text { T6IR4.0 } & 490 & 9.48 & 2.70 & 15 & 853 & 371 & 1567 \\ \text { T62R 4.0 } & 490 & 8.63 & 2.68 & 15 & 853 & 460 & 1586\end{array}$

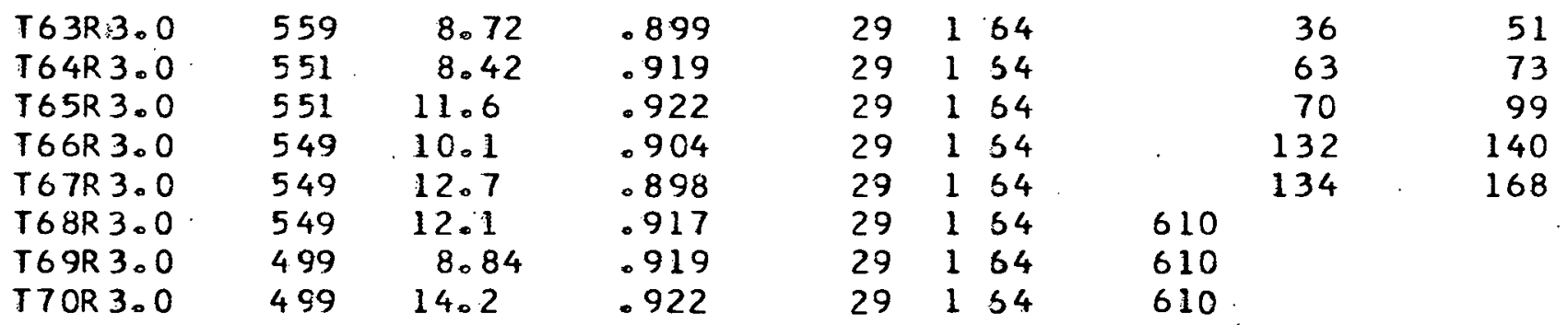

* The multiple injection dogs were male beagles born in Davis, California but injected in our laboratory. Each was injected 6 times over a 280 day period with 56 days between each injection. Each $\mathrm{Ra}^{226}$ injection was $20.0 \mu \mathrm{C}$ for the dogs Tl7R5H - T22R5H; $6.41 \mu \mathrm{c}$ for T23R4H - T27R4H; and $2.16 \mu \mathrm{C}$ for T28R3H. Tabulated for each dog are his age at lst injection, his average weight during the injection period, total $\mu \mathrm{c} /$ average weight, the date of lst injection, the time from lst injection to death, and sum of the skeletal doses computed from each injection to death.

T35R3J also received $99 \mu \mathrm{C} \mathrm{Sr}^{85}$.

T39R0.0 has been reassigned and is now MI2M0.0. 
DOG

NUMBER

COMMENTS ON DEAD DOGS

T53R 5.0 ISPECIAL STUDY T54R5.0 SPECIAL STUDY T55R:5.0 SPECIAL STUDY T56R5.0 SPECIAL STUDY

T57R.4.0 SPECIAL STUDY T58R4.0 SPECIAL STUDY T59R4.0 SPECIAL STUDY T6OR 4.0 SPECIAL STUDY T6IR4.0 SPECIAL STUDY T62R4.0 SPECIAL STUDY

T63R3.0 SPECIAL STUDY T64R3.0 SPECIAL STUDY T65R3.0 " SPECIAL STUDY T66R 3.0 SPECIAL STUDY T67R3.0. SPECIAL STUDY T 68 R 3.0 $T 69 R 3.0$

TTOR 3.0 
B. PLUTONIUM-239

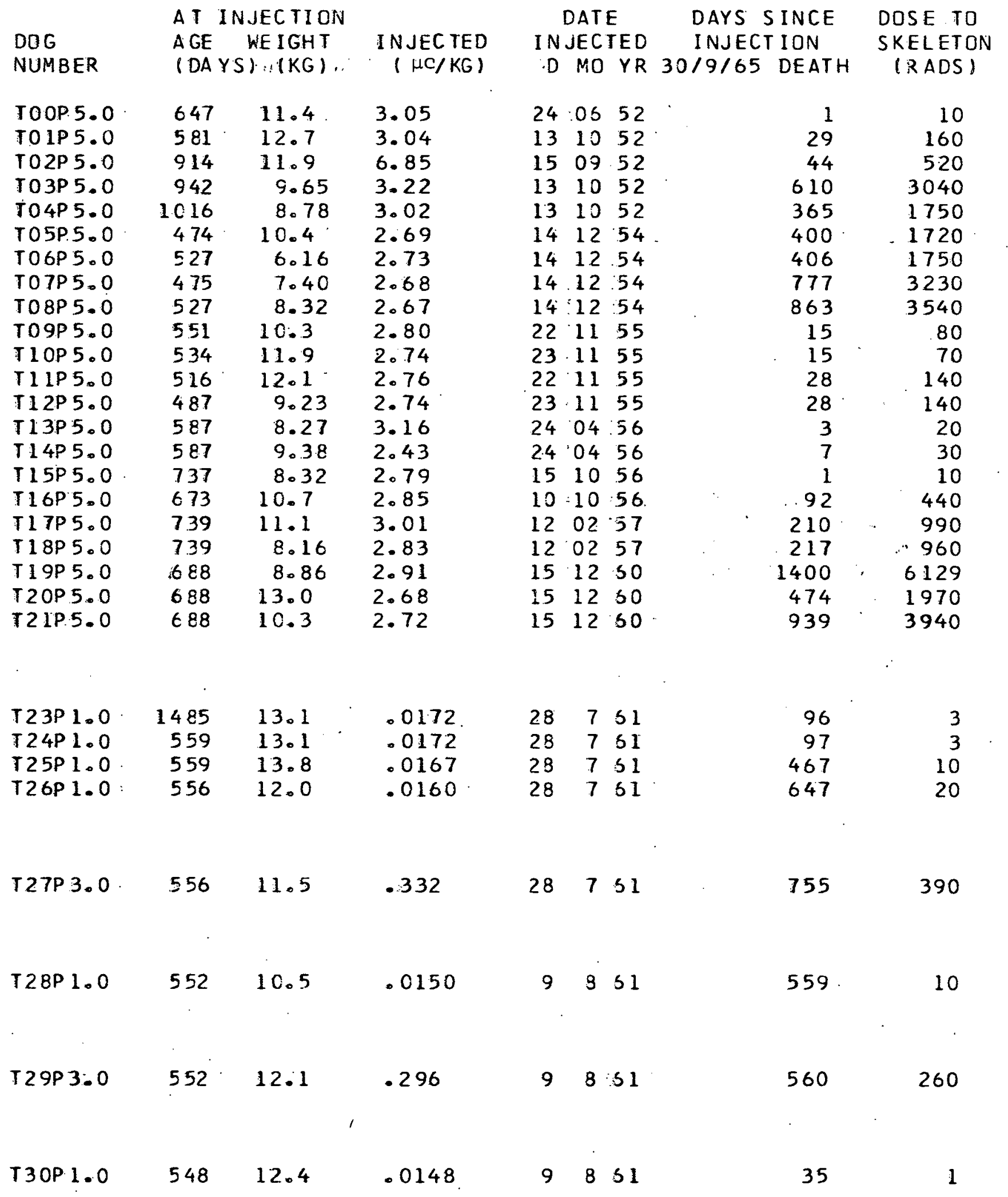


DOG

NUMBER

COMMENTS ON DEAD DOGS

TOOP.5.0 SPECIAL STUDY

TO1P5.0: SPECIAL STUDY

TO2P5.0 SPECIAL STUDY

TO3P5.0 SPECIAL STUDY

T04P.5.0 SPECIAL STUDY

TO5P 5.0 SPECIAL STUDY

TO6P5.0 SPECIAL STUDY

T07P5.0: SPECIAL STUDY

TO8P5.0 SPECIAL STUDY

TO9P5.0 SPECIAL STUDY

TIOP5.0 SPECIAL STUDY

TI1P5.0 SPECIAL STUDY

T12P5.0 SPECIAL STUDY

T13P5.0 SPECIAL STUDY

T14P.5.0 SPECIAL STUDY

T15P:5.0 SPECIAL STUDY

T16P5.0 SPECIAL STUDY

T17P5.0 SPECIAL STUDY

T18P5.0 SPECIAL STUDY

T19P5.0 - OSTEOSARCOMA,BLCOD DYSCRASI A,LIVER DEGENERAT ION

T2OP5.0 LIVER DEGENERAIION, ASCITES + THROMBOCYTOPENIA

T21P5.0 TOXIC NEPHRITIS + LIVER DEGENERATION

T23P1.0: SPECIAL STUDY

T24P1.0 SPECIAL STUDY

T25P1.0 SPECIAL STUDY

T26P1.0 SPECIAL STUDY

T27P3.0: SPECIAL STUDY

T28P1.0 SPECIAL STUDY

T29P3.0 SPECIAL STUDY

T3OP.1.0 SPECIAL STUDY 
DOG

NUMBER

T31P 3.0

T32P 1.0

T33P1:0

T 34P 1.0

520

8.47

.0162

550

10.7

550

9.68

.0153

.0154

.303

15951

362

180

T35P 3.0

$550 \cdot 11.9$

T36P 1.0

J $37 P 1.0$

$$
544
$$

542 .

10.4:

$$
.0158
$$

15951

15. 951

274

375

746

10

15. 951

15931

.0148

.304

15961

187

90

T38P 3.0 , $\quad 489$

$$
7.96
$$

$$
.304
$$

T39P 1.0

$$
1534
$$

10.7

.0151

9.92

.0177

15951

15951

376

769

10

20

T41P5.0

T42P.5.0

T43P 5. OH

T 4 P 5.0

543

8.50

3.01

2.40

$600 \quad 14.0 \quad 2.86$

$\begin{array}{lll}517 & 12.0 & 2.72\end{array}$
$15: 7 \cdot 35$

21:955

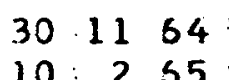

304

13

57

40: $\quad 200$

9 
DOG

NUMBER

T31P3.0: SPECIAL STUDY

T $32 P 1.0$

T33P 1.0

$\mathrm{T} 34 \mathrm{P} 1.0$

SPECIAL STUDY

SPECIAL STUDY

SPECIAL STUDY

T35P3.0 SPECIAL STUDY

T36P 1.0

$137 P 1.0$

SPECIAL STUDY

SPECIAL STUDY

T38P 3.0 S.PECIAL STUDY

T39P1.0. SPECIAL STUDY

T4OP:1.0: SPECIAL STUDY

T41P 5.0

T42P. 5.0

T43P5.OH

SPECIAL STUDY

SPECIAL STUDY

T44P 5.0 


\section{R.ADIUM-228 (ME SOTHORIUM) *}

\begin{tabular}{|c|c|c|c|c|c|c|c|c|}
\hline $\begin{array}{l}\text { DOG } \\
\text { NUMBER }\end{array}$ & $\begin{array}{l}\text { AT INJE } \\
\text { AGE WE } \\
\text { IDAYSI }\end{array}$ & $\begin{array}{l}\text { ECTION } \\
\text { EIGHT } \\
(K G)\end{array}$ & $\begin{array}{l}\text { INJEC JED } \\
(\mu \mathrm{K} / \mathrm{KG})\end{array}$ & $\begin{array}{r}\text { DATE } \\
\text { IN.JEC } \\
D \cdot M O\end{array}$ & $\begin{array}{l}\text { E } \\
\text { TED } \\
\text { YR }\end{array}$ & $\begin{array}{c}\text { DAYS } \\
\text { INJEC } \\
30 / 9 / 65\end{array}$ & $\begin{array}{l}\text { S INCE } \\
\text { T ION } \\
\text { DEATH }\end{array}$ & $\begin{array}{l}\text { DOSE TO } \\
\text { SKELETON } \\
\text { (RADS) }\end{array}$ \\
\hline $\begin{array}{l}\operatorname{TO1M4} 4.5 \\
\operatorname{TO2M4.5}\end{array}$ & $\begin{array}{l}529 \\
463\end{array}$ & $\begin{array}{l}9.13 \\
8.93\end{array}$ & $\begin{array}{l}4.23 \\
4.27\end{array}$ & $\begin{array}{l}8.09 \\
8.09\end{array}$ & $\begin{array}{l}54 \\
54\end{array}$ & : & $\begin{array}{l}314 \\
755\end{array}$ & $\begin{array}{l}1598 \\
5097\end{array}$ \\
\hline T03M5.0 & 579 & 9.15 & 10.6 & 1303 & 56 & ' & 700 & 1.5867 \\
\hline
\end{tabular}


DOG

NUMBER

COMMENTS ON DEAD DOGS

$101 M 4.5$

$T 02 M 4.5$

CAN INE DISTEMPER

SPECIAL STUDY

T03M5.0 ULCERATIVE GINGIVITIS. SEVERE: ANEMIA + CRIPPLING FRACTURE 
D. THORIUM-228 (RAOI OTHORIUM) *

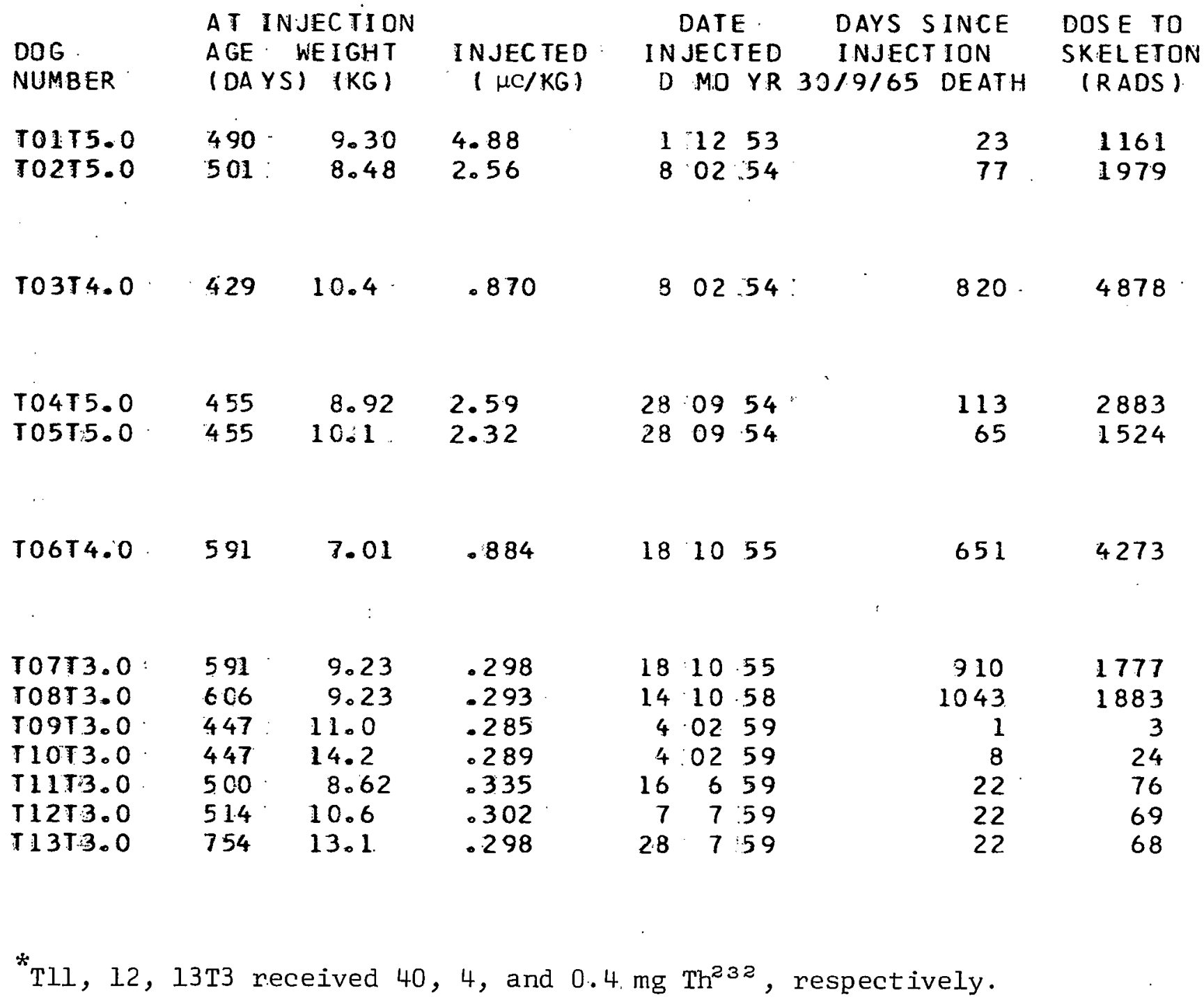


DOG

NUMBER

COMMENTS ON DEAD DOGS

TO $1 T 5.0$

DIED, SPECIAL STUDY

TO2T5.0

SPECIAL STULY

T03T 4.0

CRIPPLING FRACTURES + NEPHRITIS

T04T5.0

THR OMBOCYTOPENIA + PURPURA.

T05T5.0

NEPHRITIS, THROMBOCYTOPENIA + PURP:JRA

T06T4.0 CRIPPLING FRACTURES

TO7T3.0 SPECIAL STUDY

TO8T3.0 OSTEOSARCOMA

TO9T3.0 SPECIAL STUDY

T1OT3.0 SPECIAL STUDY

T11T3.0 SPECIAL STUDY

T12T3.0 SPECIAL STUDY

TI3T3.0 SPECIAL STUDY 
E. SIR CNTIUM-90*

\begin{tabular}{|c|c|c|c|c|c|c|c|c|c|}
\hline DOG. & $\begin{array}{l}\text { A I IN } \\
\text { AGE } V\end{array}$ & $\begin{array}{l}\text { JEC TION } \\
\text { WE IGHT }\end{array}$ & I N JEC IED & & & EED & $\begin{array}{l}\text { DAYS } \\
\text { INJEC }\end{array}$ & $\begin{array}{l}\text { INCE } \\
\text { ION }\end{array}$ & $\begin{array}{l}\text { DOSE TO } \\
\text { SKELETON } \\
\text { (RADS) }\end{array}$ \\
\hline & & $(K G)$ & & D & MO & $Y R$ & & & \\
\hline TO1SO.0 & 151 & 7.71 & & 5 & 03 & .54 & & 2 & \\
\hline $\begin{array}{l}\text { TO2S5.0 } \\
\text { T0355.0 } \\
\text { T04S5.0 } \\
\text { T05S5.0 } \\
\text { TOSS5.0 } \\
\text { TOZS5.0 }\end{array}$ & $\begin{array}{l}1.49 . \\
144 \\
151 \\
144 \\
155 \\
155\end{array}$ & $\begin{array}{l}6.85 \\
6.19 \\
7.05 \\
5.25 \\
7.01 \\
6.74\end{array}$ & $\begin{array}{l}148 . \\
1480^{\circ} \\
148 \circ \\
148 . \\
87.0 \\
87.0\end{array}$ & $\begin{array}{r}5 \\
5 \\
5 \\
5 \\
16 \\
16\end{array}$ & $\begin{array}{l}03 \\
03 \\
03 \\
03 \\
03 \\
03\end{array}$ & $\begin{array}{l}54 \\
54 \\
54 \\
54 \\
54 \\
54\end{array}$ & & $\begin{array}{r}11 \\
18 \\
41 \\
116\end{array}$ & $\begin{array}{r}626 \\
957 \\
1945 \\
4863 \\
2 \\
84\end{array}$ \\
\hline T0850.0 & 243 & 7.00 & & & & & & & \\
\hline $\begin{array}{l}\text { TO8S2.OH } \\
\text { TO9S2.OH } \\
\text { T1OS2.OH } \\
\text { T11S2.OH }\end{array}$ & $\begin{array}{l}67 \\
67 \\
67 \\
67\end{array}$ & $\begin{array}{l}3.69 \\
2.79 \\
3.11 \\
3.85\end{array}$ & $\begin{array}{l}2.74 \\
3.62 \\
3.25 \\
2.62\end{array}$ & $\begin{array}{l}27 \\
27 \\
27 \\
27\end{array}$ & $\begin{array}{l}09 \\
09 \\
09 \\
09\end{array}$ & $\begin{array}{l}55 \\
55 \\
55 \\
55\end{array}$ & & $\begin{array}{r}66 \\
66 \\
132 \\
132\end{array}$ & $\begin{array}{l}31 \\
40 \\
93 \\
75\end{array}$ \\
\hline$T 12 S 3.0$ & 593 & 10.6 & 10.5 & 11 & 09 & 57 & & 5 & 15 \\
\hline T13S4.0 & 324 & 10.5 & 19.1 & 8 & 07 & 50 & & 8 & 50 \\
\hline $\begin{array}{l}T 1455.0 \\
T 1555.0\end{array}$ & $\begin{array}{l}542 \\
595\end{array}$ & $\begin{array}{l}10.0 \\
9.43\end{array}$ & $\begin{array}{l}96.1 \\
98.4\end{array}$ & $\begin{array}{l}7 \\
7\end{array}$ & $\begin{array}{l}11 \\
11\end{array}$ & $\begin{array}{l}51 \\
51\end{array}$ & & $\begin{array}{r}9 \\
30\end{array}$ & $\begin{array}{l}175 \\
423\end{array}$ \\
\hline$T 16 S 2.0$ & 604 & 9.71 & 3.27 & 8 & 11 & 51 & & 9 & 6 \\
\hline $\begin{array}{l}T 1756.0 \\
T 1856.0\end{array}$ & $\begin{array}{l}670 \\
670\end{array}$ & $\begin{array}{l}7.18 \\
5.94\end{array}$ & $\begin{array}{l}295 . \\
302 .\end{array}$ & $\begin{array}{l}19 \\
19\end{array}$ & $\begin{array}{l}1 \\
1\end{array}$ & $\begin{array}{l}62 \\
62\end{array}$ & 1350 & 14 & 689 \\
\hline
\end{tabular}


DOG

NUMBER

COMMENTS ON DEAD DOGS

TOISO.0. SPECIAL STUDY

T0255.0 SPECIAL STUDY

TO3S5.0 SPECIAL STUDY

TO4S5.0 SPECIAL STUDY

TO5\$5.0 SPECIAL STUDY

T0655.0: SPECIAL STUDY

TO7S5.0. SPECIAL STUDY

TOBS 0 SPECIAL STUDY

TO8S2.0H

SPECIAL STUDY

TO9S2.OH SPECIAL STUDY

T1OS2.0H SPECIAL STUDY

T11S2.OH SPECIAL STUDY

T12S3.0. BREMSSTRAHLUNG. PHANTOM

T1354.0. BREMSSTRAHLUNG PHANTOM SAM MCGEE

T14S5.0. SSPECIAL STUDY

T15S.5.0 SPECIAL STUDY

T16S2.0. SPECIAL STLDY

T1756.0 LEUKDPENIA, THROMBOCYTOPENIA * PURPURA $\mathrm{T} 18 \mathrm{~S} 6.0$ 


$\begin{array}{llr} & \text { AT INJECTION } \\ \text { DOG } & \text { ACE WEIGHT } & \\ \text { NUMBER } & \text { IDAYS }(K G) & (\mu C / K G)\end{array}$

$11956.0 \quad 670 \quad 5.43284$.

$\mathrm{T} 20 \$ 4.0 \mathrm{~J}$

440

8.5428 .9

$\begin{array}{llll}T 21 S 2.5 J & 363 & 7.20 & 8.3\end{array}$
21063

13

78

DATE DAYS SINCE DOSE TO INJECTED INJECTION SKELETON D MO YR $30 / 9 / 65$ DEATH (RADS )

$19 \quad 162$

24

637

13

*T08 ... 11S2.0H were given 10 injections, 1 mc each at weekly intervals. Age is at first injection, wt. is average during the injection period, $\mu \mathrm{c} / \mathrm{kg}$ is total $\mathrm{Sr}^{90} /$ average weight, date is at first injection, days are from first injection to death, and dose is computed from mid-injection to death.

T20S4.0J received $0.5 \mu \mathrm{C} \mathrm{Sr}^{85}$.

T21S2.5J received $0.5 \mu \mathrm{c} \mathrm{Sr}^{85}$ and $600 \mu \mathrm{c} \mathrm{Sr} \mathrm{Sr}^{89}$. 
DOG

NUMBER

COMMENTS ON DEAD DOGS
T1956.0 LEUKUPENIA. THROMBOCYTOPENIA + PURPURA

T1956.0 LEUKUPENIA THROMBOCYTOPENIA + PURPURA

T20S4.0J SPECIAL STUDY

T21S2.5J SPECIAL STUDY 
F. RADIUM-224*

\begin{tabular}{|c|c|c|c|c|c|c|c|c|c|}
\hline DOG & $\begin{array}{l}A T \\
A G E\end{array}$ & $\begin{array}{l}\text { JEC II ON } \\
\text { WE IGHT }\end{array}$ & IN JEC TED & & JEC & FED & $\begin{array}{l}\text { DAYS S } \\
\text { INJECT }\end{array}$ & $\begin{array}{l}\text { INCE } \\
\text { ION }\end{array}$ & $\begin{array}{l}\text { DOSE TO } \\
\text { SKELETO }\end{array}$ \\
\hline NUMBER I & (DAYS) & $\mid(K G \mid$ & $(\mu c / K G)$ & $D$ & MO & YR & $30 / 9 / 65$ & DEATH & \\
\hline$T 0103.0 \mathrm{~J}$ & 460 & 9.55 & .875 & 26 & 3 & 53 & & $4 \mathrm{hr}$ & \\
\hline $\begin{array}{l}\text { TO2Q4.0 } \\
\text { TO3Q4.0 }\end{array}$ & $\begin{array}{l}466 \\
466\end{array}$ & $\begin{array}{l}12.0 \\
13.1\end{array}$ & $\begin{array}{l}2.91 \\
2.91\end{array}$ & $\begin{array}{l}27 \\
27\end{array}$ & $\begin{array}{l}3 \\
3\end{array}$ & $\begin{array}{l}53 \\
63\end{array}$ & $\begin{array}{l}918 \\
918\end{array}$ & & \\
\hline $\begin{array}{l}\operatorname{ro4} 45.0 \\
\operatorname{ros} 55.0\end{array}$ & $\begin{array}{l}480 \\
455\end{array}$ & $\begin{array}{l}9.55 \\
9.67\end{array}$ & $\begin{array}{l}9.71 \\
9.59\end{array}$ & $\begin{array}{l}24 \\
24\end{array}$ & $\begin{array}{l}4 \\
4\end{array}$ & $\begin{array}{l}53 \\
63\end{array}$ & $\begin{array}{l}890 \\
890\end{array}$ & & \\
\hline T06Q6.0 & 455 & 8.29 & 24.6 & 17 & 10 & 63 & & 13 & 980 \\
\hline $\begin{array}{l}\text { TO } 7 Q 5.0 \\
\text { TO } 8 Q 5.0\end{array}$ & $\begin{array}{l}465 \\
475\end{array}$ & $\begin{array}{l}11.8 \\
9.77\end{array}$ & $\begin{array}{l}9.83 \\
9.91\end{array}$ & $\begin{array}{l}6 \\
6\end{array}$ & $\begin{array}{l}11 \\
11\end{array}$ & $\begin{array}{l}53 \\
63\end{array}$ & 694 & 16 & 400 \\
\hline $\begin{array}{l}\text { TO } 924.0 \\
\text { T } 10 Q 4.0\end{array}$ & $\begin{array}{l}503 \\
503\end{array}$ & $\begin{array}{l}9.80 \\
10.3\end{array}$ & $\begin{array}{l}2.96 \\
2.96\end{array}$ & $\begin{array}{l}4 \\
4\end{array}$ & $\begin{array}{l}12 \\
12\end{array}$ & $\begin{array}{r}63 \\
63\end{array}$ & 666 & 262 & 133 \\
\hline $\begin{array}{l}T 11 Q 3.0 \\
T 12 Q 3.0 \\
T 13 Q 3.0 \\
T 14 Q 3.0\end{array}$ & $\begin{array}{l}495 \\
495 \\
495 \\
438\end{array}$ & $\begin{array}{l}9.10 \\
13.5 \\
11.3 \\
10.3\end{array}$ & $\begin{array}{l}1.02 \\
1.02 \\
1.02 \\
1.00\end{array}$ & $\begin{array}{l}4 \\
4 \\
4 \\
4\end{array}$ & $\begin{array}{l}12 \\
12 \\
12 \\
12\end{array}$ & $\begin{array}{l}53 \\
53 \\
53 \\
53\end{array}$ & $\begin{array}{l}666 \\
666 \\
666 \\
666\end{array}$ & & \\
\hline
\end{tabular}

* T01Q3.0J received $18.0 \mu \mathrm{c} \mathrm{Sr}^{85}$. 
DOG

NUMBER

TO1Q3.0J SPECIAL STUDY

T02Q4.0

TO $3 Q 4.0$

$T 0405.0$

T05Q5.0

TO6Q6.0 PURPURA HEMORRHAGICA

TO7Q5.0

TO 8Q 5.0

PUR PURA HEMORRHAGICA

$109 Q 4.0$

$T 10 Q 4.0$

STATUS EPILEPTICUS

T $11 Q 3.0$

$T 12 Q 3.0$

11303.0

$T 14 Q 3.0$ 
G. ANCI LLARY*

DOG

NUMBER

FC1A 0. 0

F02A0.O

MO $3 A 0.0$

MO4AO.O

M05AO. 0

MOGAO.O

MOTAO.O

MO8AO.O

F09A 0.0

F1OAO.O

F11A0.0

F12A0.0

F1 $3 A 0.0$

F1 $4 A 0$. 0

F15AO. 0

F1 6A.0.0

F1TAO.0

F19A0.0

F2OA0. 0

F22A0.0

M23A0.0

M24A0.0

M26A0.0

M27A0.0

M28A0.0

F32AO. 0

F $33 A 0.0$

F34A0.0

M35A0.0

M36A0.0

M37AO.O

F38A:0.0

$\mathrm{M} 42 \mathrm{AO} 0 \mathrm{O}$

F $43 A 0.0$

F $47 A 0.0$

M5 OA.0. 0

F51A0.0

F52AO:O

F54AO.O

M5 $6 A 0.0$

M5BA 0.0

M59AO. 0

M73A0.0
A T INJECTION AGE WEIGHT (DAYS) (KG)
DATE

IN JEC TED $(\mu C / K G)$
IN JECTED

DAYS SINCE

INJECT ION

D MO YR $30 / 9 / 65$ DEAT.H
DOSE TO

SKELETON

(RADS)
1383

2492

1451

3345

47.13

5266

3896

3746

3719

2605

4198

4219

4527

3777

4874

4415

2145

4166

2464

4350

1741

3074

4133

2130

3114

1990

3282

2584

529

1971

4089

3802

1265

3881

1732

2264

1089

509

3190

701

767

567

5695 
$D O G$

NUMBER

COMMENTS ON DEAD DOGS

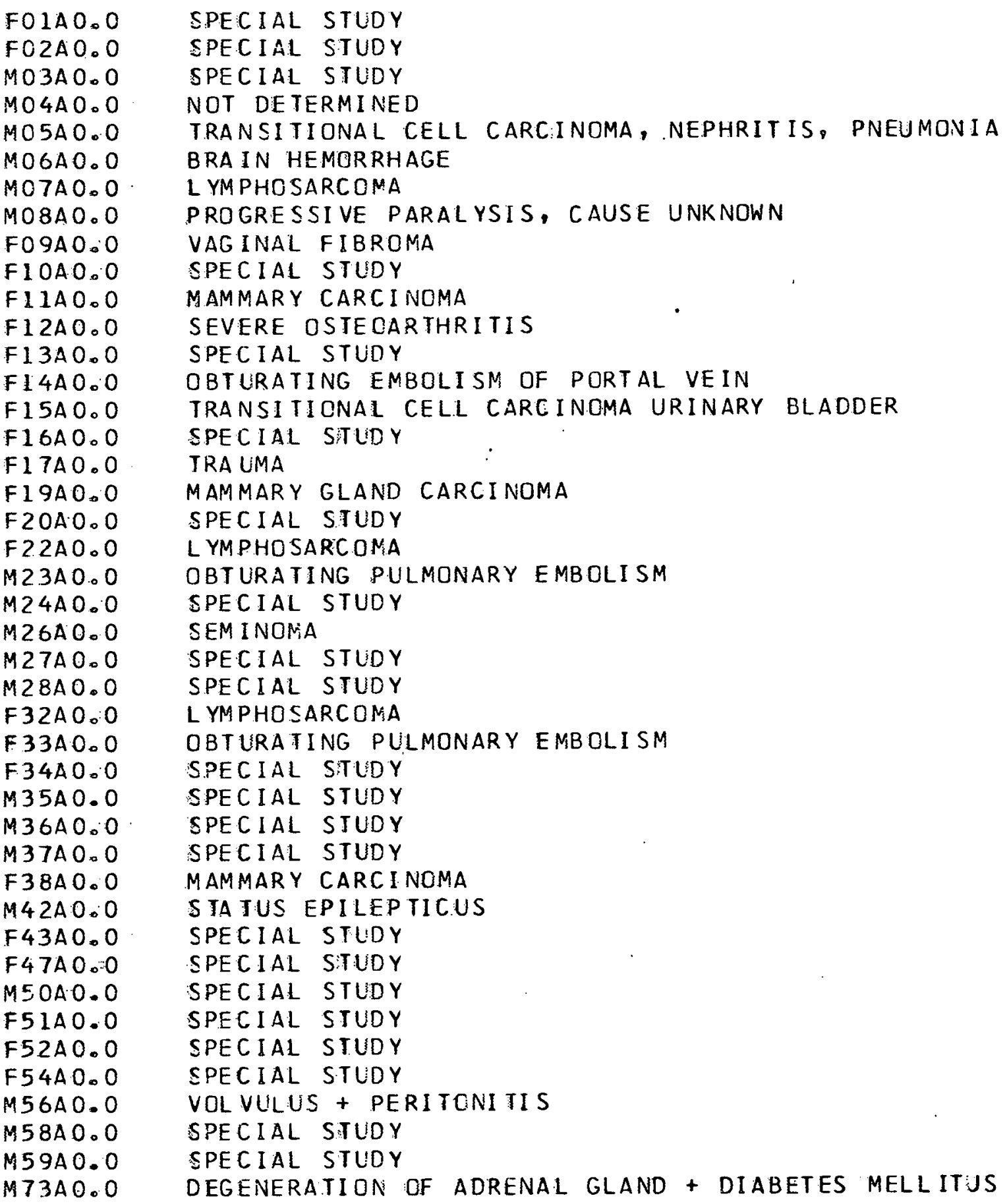


AT INJECTION

DOG

NUMBER

MB $6 A 0.0$
DATE DAYS SINCE DOSE TO IN JECTED INJECTION

D MO YR $30 / 9 / 65$ DEATH
SKELETON

(RADS)

\section{INJEC TED}

$(\mu \mathrm{C} / K G)$.

499

\footnotetext{
* Time interval shown here is the animal's age.
} 
DOG

NUMBER

M86A0.0 SPECIAL STUDY
COMMENTS ON DEAD DOGS 
AN ANALYSIS OF HEMATOLOGICAL DATA FROM BEAGLES GIVEN Ra226

US ING THE RANDOM IZED BLOCK ANALYS IS OF VARIANCE

Jean H. Dougherty; Peter J. Mikolaj, and Richard Maxwell*

\section{Introduction}

At present the major statistical analyses being carried out on the hematological data are the randomized block analysis of variance. As a result of a previous study( 1 ) of data from beagles given $\mathrm{Sr}^{90}$, it was decided that this type of analysis better fitted the experimental design rather than the one-way analysis of variance. Other methods of analysis will also be tried in the future in order to obtain the most meaningful data. Since $\mathrm{Ra}^{226}$ is the radionuclide to which the others are compared, it was decided to make the preliminary analysis on the dogs injected with radium.

\section{Methods}

A series of randomized block analyses of variance were made on the radium data at 6 month intervals from 6 months post injection through 120 months post injection. A similar type of analysis on the dose-time relationship of several hematological values was made several years ago on dogs injected with $\mathrm{Pu}^{239}, \mathrm{Ra}^{226}, \mathrm{Ra}^{228}$ and $\mathrm{Th}^{228}$ for 24 months post injection(2). Because the 1.7 level dogs were added later in the experiment, the analysis was performed on the $0,1,2,3,4$ and 5 level dogs only. An earlier study(3) indicated that there was a time trend in several hematological values; thus we could utilize only those dogs which were entered into the experiment as a block or group.

A program was written for the IBM 7040-1401 computer system at the University of Utah Computer Center. Since covariance and non-independence between paired measurements were suspected, it was decided that the

\footnotetext{
*University of Utah Computer Center.
} 
randomized block design would provide an efficient model for the experiment.

The mathematical model used was:

$$
X_{i j}=M+T+B+E
$$

where $X_{i j}$ is a measurement from the $i^{\text {th }}$ treatment and $j^{\text {th }}$ block, $M$ is overall"or grand mean, $\mathrm{T}$ is the treatment effect, $\mathrm{B}$ is the block effect, and $E$ is the random error effect: The numbers $M, T, B$ are fixed constants, and $E$ is a normally and independently distributed random variable with" mean zero and common variance $\sigma^{2}$. In this experiment "block" refers to a group of dogs injected at the same time and "treatment groups" are the several dose levels used:

An important assumption for this model is that the variances of -all treatment groups must be equal. This is a very critical point since one would suspect that high doses of radiation may appreciably alter variances within treatment groups.

The null hypothesis for this design states there are no differences in treatment means. This hypothesis is accepted or rejected depending on whether the calculated F-ratio for treatments is smaller or larger than the tabulated value. 'If the null hypothesis is rejected, we must: find a way to determine where the differences are among the treatment means. Since T-tests are invalid when one his more than two groups, we decided to use a multiple range test (discussed further below).

Because of the possibility of a large amount of biological varia-. bility, it was decided to include in the program a test for homogeneity of variances. The standard test is Bartlett's(4) which compares the'individual variances to the average variance. After noting that the Bartlett's approached the significant reference $X^{2}$ in the intervals immediately . following irijection of the radionuclide, we decided to add a second test, 
Cochran's(5), which compares the largest group variance to the sum of all the group variances. Thus, we would have two methods testing whether the data met the assumptions of the randomized block analysis.

A third consideration for this analys is was the handling of missing data. If a dog was found to have a clinical illness on the day it was scheduled for testing, we felt that the hematological values would reflect clinical illness not radiation and should not be included. Thus, when the situation warranted, the card was pulled from the 6 months data. Also, a value could be missing due to the death of the dog. Because the randomized block method requires a measurement for each cell, another routine was included in the program. As mentioned in the report on the dose-time relationship (2), the missing data were estimated by the Yates(6) method. In order to reduce the possibility of error, when a value is estimated the error degrees of freedom are reduced by one. In general, this increases the denominator of the $F$ ratio, resulting in a smaller $F$ value. To investigate just how many values could be estimated by this method, runs were made where first all the values were known, and then measurements were removed up to $50 \%$ of the entire deck. For the most part $25 \%$ of the data could be estimated without appreciably increasing or decreasing the number of significant differences. Thus, we decided upon using $25 \%$ as the maximum number of total variates to be estimated and arbitrarily set $50 \%$ and 66-2/3\%, respectively, as the block and treatment maxima. When the number to be estimated for individual block or treatment exceeded the mentioned limits, the entire group was eliminated. In passing it was noted that the group variances became more homogeneous as the number of missing values increased.

A final routine that was written into the program was the multiple range test mentioned previously. To allow for future revisions in the 
analysis, the test chosen was the Duncan(7). By simply changing an information card with different table values, one could perform the 'Student-Newman-Keuls' multiple range test with different probabilities of Type I and II errors. $\therefore$. We chose the Duncan for this series of experimental analyses because we wished to minimize the possibility of rejecting a difference that was true.

In order to obtain a better picture of the overall experiment, a second computer program has been written. This program graphs by dose level (up to four levels of a radionuclide on one graph) the monthly means for all hematological values containing three or more dogs per group. Thus, all the data available can be utilized in providing a graphic representation of the experiment.

Table I. Sample Size of Radium Randomized Block Analyses

\begin{tabular}{ccccc}
\hline $\begin{array}{c}\text { Months } \\
\text { Post Injection }\end{array}$ & $\begin{array}{c}\text { Total } \\
\text { Observations }\end{array}$ & $\begin{array}{c}\text { Number of } \\
\text { Blocks }\end{array}$ & $\begin{array}{c}\text { Number of } \\
\text { Dose Levels }\end{array}$ & $\begin{array}{c}\text { Observations } \\
\text { Estimated }\end{array}$ \\
\hline 6 & 72 & 12 & 6 & 4 \\
12 & 72 & 12 & 6 & 4 \\
18 & 66 & 11 & 6 & 3 \\
24 & 72 & 12 & 6 & 3 \\
30 & 72 & 12 & 6 & 5 \\
36 & 72 & 12 & 6 & 9 \\
42 & 72 & 12 & 6 & 9 \\
48 & 60 & 12 & 5 & 4 \\
54 & 60 & 12 & 5 & 13 \\
60 & 48 & 12 & 4 & 3 \\
66 & 48 & 12 & 4 & 6 \\
72 & 48 & 12 & 4 & 10 \\
78 & 48 & 12 & 4 & 10 \\
84 & 44 & 11 & 4 & 10 \\
90 & 30 & 10 & 3 & 3 \\
96 & 36 & 12 & 3 & 5 \\
102 & 36 & 12 & 3 & 9 \\
108 & 30 & 10 & 3 & 8 \\
114 & 18 & 6 & 3 & 4 \\
120 & 12 & 4 & 3 & 2 \\
\hline
\end{tabular}


Results

The number of dogs. at the various. six monthly intervals is presented in Table I: The sample size decreases with time post injection due to the death of some of the dogs. In a . Eew instances where a large number of values were estimated (i.e., 42 months post injection, etc.) the majority of missing values came from the highest dose level at that point, since only a few dogs at this dose level were alive at this time. The 0,1 and 2-levels were analyzed through 120 months post injection, the 3-level through 84 months, the 4-level through 54 months and the 5level through 42 months.

Table II. Actual Values of Lymphocytes of Dogs Injected with $\mathrm{Ra}^{226}$ at 6 Months Post Injection

\begin{tabular}{|c|c|c|c|c|c|}
\hline \multicolumn{6}{|c|}{ Dose Level } \\
\hline 0 & 1 & 2 & 3 & 4 & 5 \\
\hline 3.240 & 3.030 & 4.500 & 2.520 & 1.890 & 1.460 \\
\hline 4.970 & 5.490 & 3.920 & 3.670 & 3.220 & 1.550 \\
\hline 2.840 & $4.106 *$ & $3.557 *$ & 3.900 & $2.380^{\circ}$ & 2.140 \\
\hline 6.770 & 4.010 & 3.360 & 4.720 & 4.610 & 4.250 \\
\hline 2.960 & 5.290 & 3.110 & 3.150 & 2.390 & 1.570 \\
\hline 5.260 & 5.130 & 3.420 & 4.660 & 2.360 & 2.020 \\
\hline 2.050 & 3.730 & 3.220 & 1.770 & 2.180 & 1.140 \\
\hline 2.810 & 5.120 & 2.820 & 3.140 & 2.320 & 2.120 \\
\hline 4.970 & 3.860 & 4.180 & 5.220 & 2.170 & 2.090 \\
\hline 4.070 & 3.300 & 5.490 & 2.740 & 2.170 & 1.390 \\
\hline 3.630 & 4.650 & 4.000 & 4.570 & 2.580 & 2.224 \\
\hline 4.450 & 3.920 & 3.470 & 3.970 & 0.670 & 1.634 \\
\hline
\end{tabular}

* Underlined values are those which were estimated.

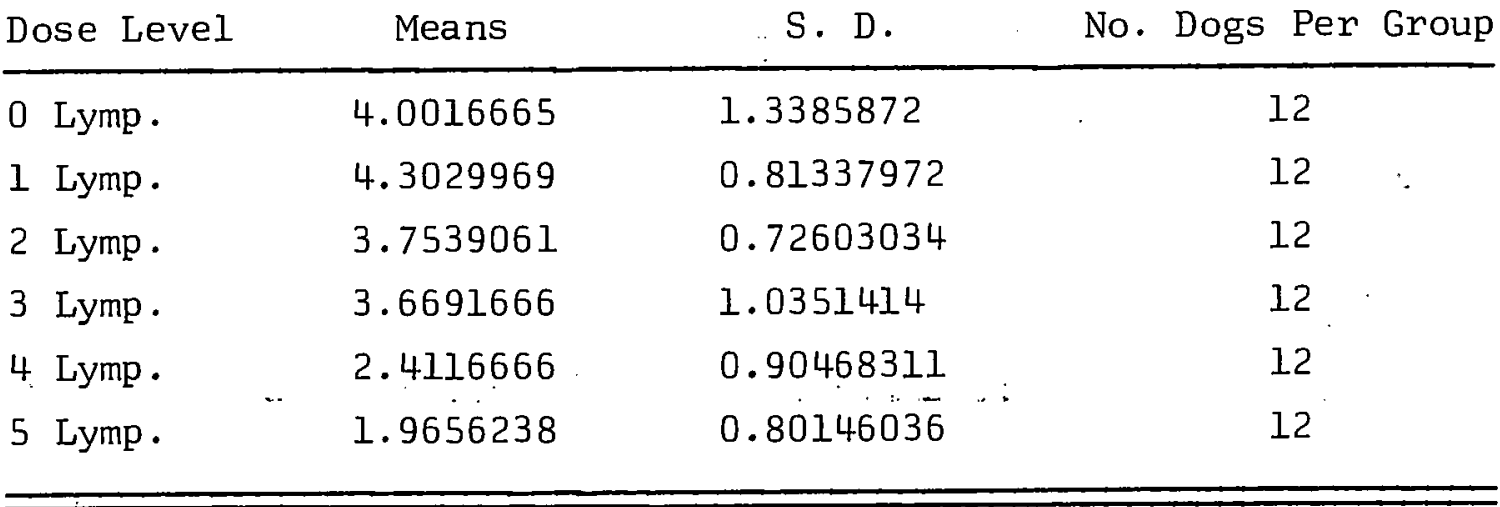


Table III. Results of Analysis on Lymphocytes of Dogs Injected with $\mathrm{Ra}^{226}$ at 6 Months Post Injection (Similar information is available for all analyses)

\begin{tabular}{lrrr}
\hline \hline Source & df & Sum of Squares & Mean Square \\
\hline Total & 67 & 113.37933 & 10.547667 \\
Treat & 5 & 52.738335 & 2.1401311 \\
Block & 11 & 23.541443 & 0.72744227 \\
Error & 51 & .37 .099556 &
\end{tabular}

F-Ratio for Treatments $=14.5(5,51)$

Tabled Value of F for Treatments $=\quad 2.4000000$

F-Ratio for Blocks $=\quad 2.94(11,51) \quad$ F.05 $=1.98$

Bartletts Test for Homogeneity of Variances
Corrected $\mathrm{x} 2=$
5.6949742
(5)
$.40>\mathrm{P}>.30$

Cochrans Test for Homogeneity of Variances
$S=$
0.32502645
$(6,12)$
P.05 $=0.3568$

Standard Error=

0.2462

$\mathrm{df}=51$

Means Being Analyized

5 Lymp.

1.9656238

4 Lymp.

2.4116666

3 Lymp.

3.6691666

2 Lymp:

3.7539061

0 Lymp.

4.0016665

I Lymp.

4. 3029969

5 Lymp - is: NE TO

3 Lymp.

2 Lymp.

0 Lymp.

1 Lymp.

4 Lymp. is NE TO

3 Lymp .

2 Lymp.

0 Lymp .

1 Lymp. 
Table. II shows a typical run from the computer on the lymphocyte values at 6 months post injection starting with the individual measurements. The values that have been underlined were those which were estimated. The mean lymphocyte values, standard deviations, and numbers per group are found in the lower half of the table. The remainder of the various tests performed by the computer are seen in Table III. If the $\mathrm{F}$ ratio found from the analysis is larger than the reference value, the program calculates the standard error of the mean and then continues with the Duncan's multiple range test. The degrees of freedom for the standard error of the mean are the same as those used in the error portion of the analysis. Thus, when a number of values are estimated, the resulting standard error is increased and a larger difference is needed before it can be called significant.

Tables IV, V, and VI present the months post injection where there are significant differences between dose levels (at the .01 or .05 level of significance) for mean values of the total white count (WBC), polymorphonuclear leukocytes (pmns) and lymphocytes, respectively. In all cases with the exception of the pmns in 5-level dogs at 42 months there was a decrease in numbers of cells rather than an increase. The volume of packed red cells (VPRC), sedimentation rate, reticulocytes, monocytes, eosinophils, and immature pmns were also analyzed in the same way but because of lack of space were not included in the tables. The only significant change in red cells occurred in the 5-level dogs as seen by a drop in VPRC and a rise in sedimentation rate at 18 through 42 months and an increase in reticulocytes at 30 and 36 months post injection. There was a depression in monocytes and eosinophils in the 3, 4 and 5level dogs which occurred about the same time as the decrease in pmns. As expected, the largest number of significant differences are found 
Table IV. Months Post Injection Where There are Significant Differences Between Dose Levels in WBC of Dogs Injected with $\mathrm{Ra}^{226}$

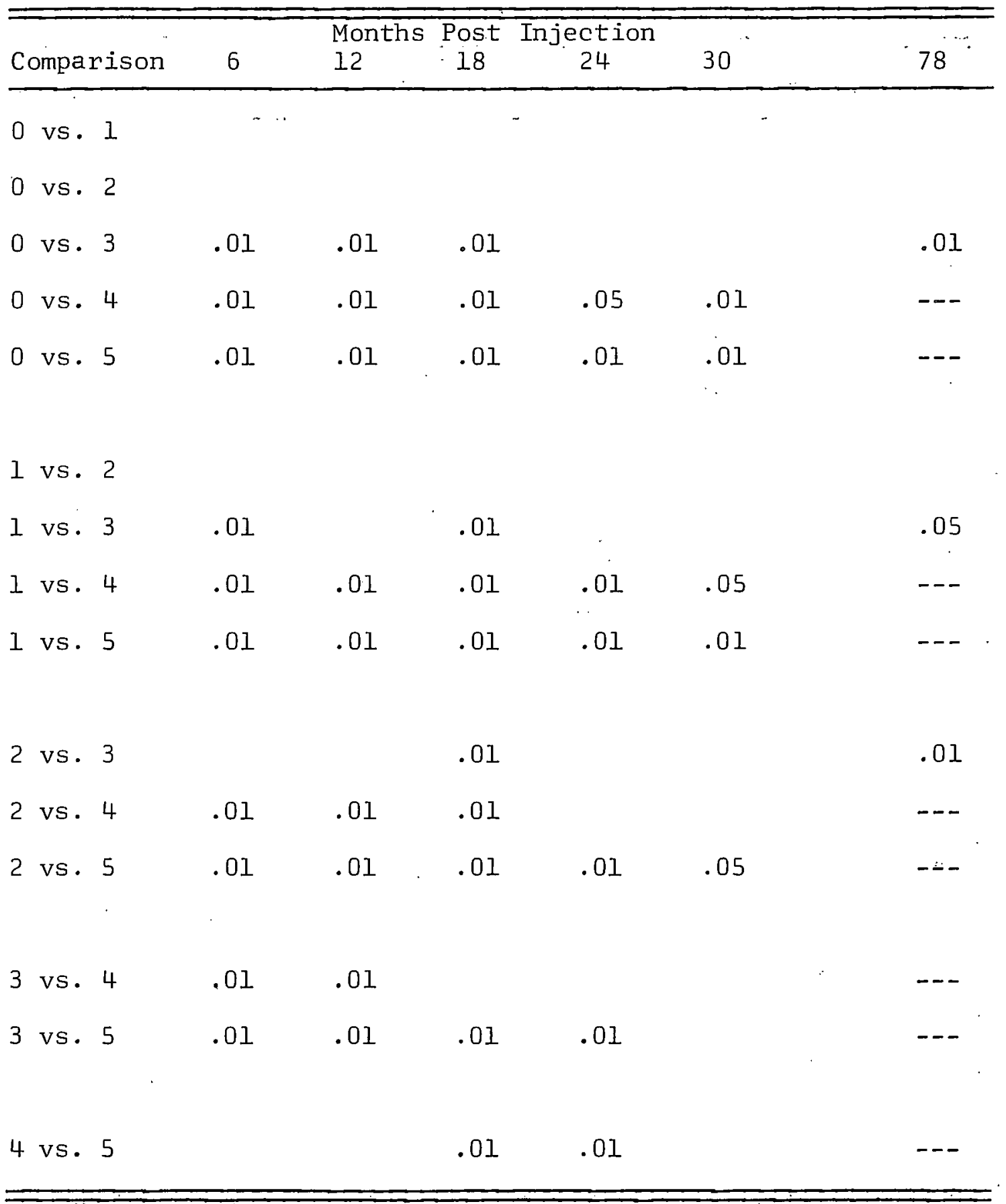


Table V. Months Post Injection Where There are Significant

Differences Between Dose Levels in pmns of Dogs Injected with $\mathrm{Ra}^{226}$

\begin{tabular}{|c|c|c|c|c|c|c|c|c|c|}
\hline & & & iths & it Ir & tio & & & & \\
\hline Comparison & 6 & 12 & 18 & 24 & 30 & $\approx$ & 42 & $\approx$ & 66 \\
\hline 0 vs. 1 & & & & & & & & & \\
\hline 0 vs. 2 & .01 & & & & & & & & \\
\hline 0 vs. 3 & .01 & .05 & .05 & & & & & & .05 \\
\hline $0 \mathrm{vs} .4$ & .01 & .01 & .05 & & & & & & -- \\
\hline 0 vs. 5 & .01 & .01 & .01 & .01 & $: 01$ & & .05 & & -- \\
\hline
\end{tabular}

1 vs: 2

l vs. $3 \quad .05 \quad .01$

$\begin{array}{lllll}\text { l vs. } 4 & .01 & .01 & .01 & .05\end{array}$

$\begin{array}{llllllll}1 & \text { vs. } 5 & .01 & .01 & .01 & .01 & .01 & .05 \uparrow\end{array}$

2 vs. 3

.01

.05

2 vs. 4

$.05 \quad .01 \quad .01$

2 vs. 5

$\begin{array}{llll}.01 & .01 & .01 & .01\end{array}$

$.05 \uparrow$

$\begin{array}{lll}3 \text { vs. } 4 & .05 \quad .05\end{array}$

3 vs.

$.05 \quad .01$

$.05 \quad .01$

$.05 \uparrow$

4 vs. 5

.05

.05

.05 .1 
Table VI. Months Post Injection Where There are Significant

Differences Between Dose Levels in Lymphocytes of Dogs Injected with $\mathrm{Ra}^{226}$

\begin{tabular}{|c|c|c|c|c|c|c|c|c|c|c|c|c|c|c|c|}
\hline \multirow{2}{*}{ Comparison } & \multirow[b]{2}{*}{6} & \multirow[b]{2}{*}{12} & \multirow[b]{2}{*}{18} & \multirow[b]{2}{*}{24} & \multirow{2}{*}{\multicolumn{3}{|c|}{$\begin{array}{cr}\text { Months } & \text { Post } \\
36 & 42\end{array}$}} & \multicolumn{2}{|c|}{ Injection } & \multirow[b]{2}{*}{$\approx$} & \multirow[b]{2}{*}{72} & \multirow[b]{2}{*}{78} & \multirow[b]{2}{*}{84} & \multirow[b]{2}{*}{$\approx$} & \multirow[b]{2}{*}{99} \\
\hline & & & & & & & & $\approx$ & 54 & & & & & & \\
\hline 0 vs. 1 & & & & & & & & & & & & & & & \\
\hline 0 vs. 2 & & & & & & & & & & & & & & & \\
\hline 0 vs. 3 & & .05 & .05 & & & & & & & & .05 & .05 & .05 & & $\therefore-$ \\
\hline $0 . v s \cdot 4$ & .01 & .01 & .01 & .01 & .01 & .05 & .05 & & .01 & & --- & $-\cdots$ & $=--$ & & --- \\
\hline 0 vs. 5 & .01 & .01 & .01 & .01 & .01 & .05 & .01 & & $\ldots$ & & -- & --- & -- & & -- \\
\hline 1 vs. 2 & & & & & & & & & & & & & & & .05 \\
\hline 1 vs. 3 & & .05 & & & . & & .05 & & & & .05 & .05 & .05 & & --- \\
\hline 1 vs. 4 & .01 & .01 & .01 & .01 & .05 & .05 & .01 & & .01 & & -- & --- & --- & & $-\ldots-$ \\
\hline 1 vs. 5 & .01 & .01 & .01 & .01 & .01 & .05 & .01 & & --- & & --- & --- & --- & & --- \\
\hline 2 vs. 3 & & & & & & & & & & & .05 & .05 & & & --- \\
\hline 2 vs. 4 & .01 & .01 & .05 & .05 & & .05 & & & .01 & & -- & $\cdots$ & $-\cdots$ & & --- \\
\hline 2 vs. 5 & .01 & .01 & .01 & .01 & .05 & .05 & .01 & & --- & & --- & -- & --- & & --- \\
\hline 3 vs. 4 & .01 & .01 & & .05 & & .05 & & & & & --- & --- & -- & & --- \\
\hline 3 vs. 5 & .01 & .01 & .01 & .01 & .01 & .05 & .01 & & -- & & --- & -- & -- & $\cdot$ & -- \\
\hline 4 vs. 5 & & & .05 & .05 & & & .05 & & --- & & --- & --- & --- & & --- \\
\hline
\end{tabular}


in the early monthly periods following injection of the radionuclide. The WBC, which is made up largely of pmns, is depressed at the 3,4 and 5-level from 6 through 18 months. This depression continues through 30 months in the 4 and 5-level dogs. There is a significant fall in pmns at the 2-level at 6 months with recovery by 12 months post injection. There is no change in any hematological values at the l-level. The pmn depression (Table $V$ ) at the 5-level continues through 30 months and these cells exceed the normal range at 42 months (i) when the dogs become terminal. The lymphocytes (Table VI) are affected through the 3-level but go down more slowly and stay depressed for a longer interval of time (through 84 months) after injection.

\section{Discussion and Summary}

In writing the program for the randomized block analysis of variance on the $\mathrm{Ra}^{226}$ data, a major problem that arose was how to deal with the missing data. The test for block significance indicated that during the periods following injection up to about 36 months there was a component of variance due to the way the animals were entered into the experiment that should be removed before testing for mean differences. For this period, then, the Yates correction provided the best method of handling the data. During this early post injection time period, there were relatively few values that were missing, and thus we felt it better to estimate the missing cell rather than eliminate the row or block containing the missing value.(s). Once the high level irradiated dogs were dropped from the analysis, however, there are two choices that might be made; (1) continue with the Yates estimation, or (2) go to the single classification analysis of variance when there are missing data. We chose the former method because the randomized block analysis provides greater sensitivity in that the error mean square is partitioned into the 
components of block and error rather than into one component alone. We feel that most meaningful results are obtained when the analysis is consistent over the entire period tested.. Thus, we kept the randomized block procedure because it provides more sensitivity in obtaining real differences.

Although the results presented above give a general picture of the depression and recovery of blood cells following irradiation, the fact that the analyses are performed on all dose levels at 6 month intervals leaves a.considerable span of time without data by this method of analysis. However, there are data available from the 3-level dogs at 3 month intervals and from 4 and 5 -level dogs at monthly intervals which could be utilized for comparison to controls (0-level dogs) if we had a method for estimating the values of the 0 -level dogs between the 6 month intervals when counts were actually made. One method which is being tried at the present time by computer programming is to fit a curve to the actual data by running a series of polynomial equations on each hematological trait on a dog and choosing the equation (up to a 7 th degree polynomial) which best describes the data points. The missing monthly values can then be generated from the curve and inserted into the randomized block program.

\section{References}

1. Dougherty, J. H., Rosenblatt, L. S., and Mikolaj, P. J., A comparison of two methods of analyzing the hematological data from Strontium-90 injected beagles. Radiobiology Laboratory, University of Utah Annual Report C00-119-232, 165-187 (March 1965) •

2. Dougherty, J. H., Rosenblatt, L. S., and Welch, Q. B., A preliminary analysis of the dose-time relationship of selected hematological values. Radiobiology Laboratory, University of Utah Annual Report C00-224, 46-56 (September 1961). 
3. Dougherty, J. H. and Rosenblatt, L. S., Changes in the hemogram of the beagle with age. J. Gerontology, 20, pp. 131-138 (1965ु)

4. Ostle, B., Statistics in Research, Ames, Iowa: Iowa State College Press, 1954, pp. 242-244.

5. Dixon, W. J., and Massey, F. J., Introduction to Statisticál Analysis, New York: McGraw Hill Book Company, 1951, p. 147.

6. Cochran, G., and Cox, G. M., Experimental Designs, New York: John Wiley and Sons, Inc., pp. 110-112.

7. Hunter, H. L., Critical values for Duncan's new multiple range test. Biometrics, 16, pp. 67i-685 (1960). 


\title{
SOFT TISSUE RETENTION OF Sr9 $\mathrm{S}^{\circ}$ IN THE BEAGLE
}

\author{
David R. Atherton and Betsy J. Stover
}

\begin{abstract}
The concentrations of $\mathrm{Sr}^{90}$ found in the liver, kidneys, and spleen of beagles, measured at various times following injection, decrease with increasing time and, after a. year or so, appear to decrease at the same rate as does whole body retention. These results are compared with similar measurements made on soft tissues from beagles injected with $\mathrm{Ra}^{226}$ and with the calcium concentrations measured in the soft tissues of humans.

\section{Introduction}

In previous publications from this laboratory $(1,2,3,4,5)$ various features of the studies of $\mathrm{Sr}^{\circ 0}$ in the beagle have been reported. In those reports it has been said that $\mathrm{Sr}^{90}$ deposits almost exclusively in the skeleton. The purpose of this brief report is to tabulate our findings relative to the retention of $S_{r}^{90}$ by non-mineralized tissue. Due to restrictions of time available for such studies, the tissues selected for analysis were limited to the liver, kidneys, and spleen, paralleling. the studies done on the soft tissue retention of $\mathrm{Ra}^{226}(6)$.

\section{Materials and Methods}

$\mathrm{Sr}^{90}$ was given to the dogs in these studies in a single I.V. injection in early adulthood as described previously(5). Weighed fractions of the tissues involved were obtained at autopsy. Sr9o analysis was done on the tissues as described by Atherton (7).

\section{Results}

The percent of the injected dose (corrected for physical decay) retained per gram of each of the three tissues studied is listed in Table 1. Also shown are the animal's identification number, and the interval between injection and death. The tabulation is arranged in order of increasing time interval and it is apparent that the concentration of $\mathrm{Sr}^{\circ 0}$ in these tissues tends to decrease with increasing time. 
The concentrations in spleen and liver appear to be about the same while that of the kidney appears to be higher than either except in the case of Flls5 where the concentration in the spleen is shown to be high. . It is possible that this high spleen concentration is a result of mislabeling samples rather than metabolic anomaly.

Table 1. Concentration of $\mathrm{Sr}^{90}$ in Liver, Kidneys, and Spleen

\begin{tabular}{|c|c|c|c|c|}
\hline Dog & $\begin{array}{l}\text { Interval: } \\
\text { Injection } \\
\text { To Death } \\
\text { (days) }\end{array}$ & $\begin{array}{l}\frac{\% \text { of Inj }}{g n} \\
\text { Liver }\end{array}$ & $\begin{array}{l}\text { od Dose } x \\
\text { Kissue } \\
\text { Kidneys }\end{array}$ & $\begin{array}{l}\times 10^{6} \\
\text { Spleen }\end{array}$ \\
\hline FISI & 1 & 1800 & & 1100 \\
\hline TI4S5 & 9 & 66 & 190 & 50 \\
\hline T16S2 & 9 & 44 & 240 & 350 \\
\hline T15S5 & 30 & 12 & 100 & 56 \\
\hline M6S5 & 35 & 20 & 30 & 18 \\
\hline M2S5 & 255 & 3.6 & & \\
\hline FlS5 & 960 & 2.7 & 5.6 & 1.6 \\
\hline M10S5 & 990 & 5.6 & 7.1 & 0.21 \\
\hline M6S5A & 1021 & 2.1 & 9.6 & 2.4 \\
\hline F7S5 & 1129 & 1.1 & 10 & 2.1 \\
\hline Ml2S5 & 1165 & 0.31 & 2.4 & 1.0 \\
\hline F4S5 & 1448 & 0.19 & 2.6 & 0.37 \\
\hline F $8 S 5$ & 1469 & 1.2 & 7.6 & 1.2 \\
\hline FIIS5 & 1667 & 0.25 & 0.32 & 6.7 \\
\hline M2S5A & 1740 & 0.35 & 3.0 & 1.1 \\
\hline F9S5 & 1982 & 0.47 & & 0.54 \\
\hline M2S 4 & 2093 & 0.52 & 4.7 & 0.58 \\
\hline F $8 S 4$ & 2206 & 0.35 & 4.7 & 0.46 \\
\hline M3S5 & 2256 & 0.33 & 3.8 & 0.52 \\
\hline M3S4 & 2781 & 0.27 & 5.3 & 0.61 \\
\hline FIS 4 & 3682 & 0.11 & 2.8 & 1.1 \\
\hline
\end{tabular}

In Table 2 the contribution to whole body' retention made by these three tissues is shown. Alsö presented is animal identification and the 
time interval, as in Table 1 , and the whole body retention of these animals which was measured by C. W. Mays and R. D. Lloyd of our physics group and to whom we are indebted for making their results available to us.

Table 2. Comparison of Whole Body to Soft Tissue Retention of $\mathrm{Sr}^{90}$

\begin{tabular}{|c|c|c|c|}
\hline Dog & $\begin{array}{l}\text { Interval: } \\
\text { Injection } \\
\text { To Death } \\
\text { (days) }\end{array}$ & $\mathrm{R}_{(\mathrm{M})}^{\mathrm{a}}$ & $\frac{\mathrm{R}^{\mathrm{b}}(\mathrm{LKS})}{\mathrm{R}_{(\mathrm{M})}} \times 10^{3}$ \\
\hline TI4S5 & 9 & 0.344 & 74 \\
\hline Tl6S2 & 9 & 0.380 & 89 \\
\hline T15S5 & 30 & 0.242 & 32 \\
\hline M6S5 & 35 & $0.318^{C}$ & 27 \\
\hline FIS5 & 960 & $0.128^{C}$ & 9.7 \\
\hline MIOS 5 & 990 & 0.136 & 18 \\
\hline M6S5A & 1021 & $0.126^{c}$ & 9.9 \\
\hline F7S5 & 1129 & 0.163 & 5.2 \\
\hline M12S5 & 1165 & 0.113 & 3.8 \\
\hline F 4S5 & 1448 & 0.0844 & 2.4 \\
\hline F $8 S 5$ & 1469 & 0.129 & 6.3 \\
\hline Fl1S5 & 1667 & 0.102 & 2.8 \\
\hline M2S5A & 1740 & 0.121 & 3.5 \\
\hline M2S4 & 2093 & 0.113 & 4.3 \\
\hline F8S4 & 2206 & 0.137 & 3.0 \\
\hline M3S5 & 2256 & 0.102 & 3.7 \\
\hline M3S4 & 2781 & 0.0712 & 7.3 \\
\hline \multirow[t]{2}{*}{ FIS4 } & 3682 & 0.0694 & 2.9 \\
\hline & & Average & $5.9 \pm 4.3^{d}$ \\
\hline
\end{tabular}

${ }^{a_{R}}(M)=$ Measured fractional whole body retention.

$\mathrm{b}_{\mathrm{R}}$ (LKS) $=\begin{gathered}\text { Measured percent retention found in the liver, kidneys and } \\ \text { spleen. }\end{gathered}$

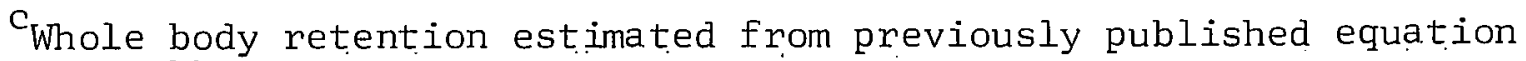
for $\mathrm{Sr}^{\circ} \mathrm{O}$ reterition in the beagle (8).

$\mathrm{d}_{\text {Mean }} \pm 1$ standard deviation of values from 960 days onward. 


\section{Discussion}

As preliminary studies indicated(4) the soft tissue retention of $\mathrm{Sr}^{90}$, as reflected by its retention in the liver, kidneys, and spleen, is very small, and decreases with time. Perhaps the most interesting feature of the data presented here is that the contribution these three tissues make to whole body retention seems to remain somewhat constant from sometime prior to 1000 days onward. In as much as whole body retention of $\mathrm{Sr}^{90}$ is essentially skeletal retention and in view of the rapid "wash out" of $\mathrm{Sr}^{90}$ from soft parts it appears that $\mathrm{Sr}^{\circ 0}$ associated with soft tissues at long times following injection is controlled by the animal's $\mathrm{Sr}^{90}$ reservoir (the skeleton) and diminishes as the reservoir is depleted.

For comparative purposes data relative to the soft tissue retention of $\mathrm{Ra}^{226}$, previously presented (6), has been treated the same as that seen in Table 2 and is shown in Table 3. Here too it appears that, from 500 days following injection and onward, the..radium found in the liver, kidneys, and spleen make a rather constant contribution to that retained by the whole body. However the contribution to whole body retention made by these three tissues is about a factor of 9 higher in the case of radium than in the case of strontium, $0.052 \pm 0.034 \%$ for radium and $0.0059 \pm$ $0.0043 \%$ for strontium.

If, as seems likely, the soft tissue retention of these two alkaline earth elements, at long times following their injection, is a reflection of bone metabolism, it would be interesting to compare these findings to calcium found in the same soft tissues. Unfortunately calcium measurements on other than mineralized tissue have not been made on the animals in these studies. However the ICRP Task Group on the Revision of the Standard Man(9) has collected a great deal of data including the 
Table 3. Comparison of Whole Body to Soft Tissue Retention of $\mathrm{Ra}^{226}$

\begin{tabular}{|c|c|c|c|}
\hline Dog & $\begin{array}{l}\text { Interval: } \\
\text { Injection } \\
\text { To Death } \\
\text { (days) }\end{array}$ & $\mathrm{R}_{(\mathrm{M})}^{\mathrm{a}}$ & $\frac{R^{\mathrm{b}}(\mathrm{LKS}) \mathrm{x}_{10}{ }^{3}}{\mathrm{R}_{(\mathrm{M})}}$ \\
\hline MIORI. 7 & 590 & 0.22 & 42 \\
\hline Ml0R5 & 825 & 0.21 & 52 \\
\hline MIR5 & 908 & 0.24 & 37 \\
\hline F8R5 & 968 & 0.22 & 73 \\
\hline F6R5 & 1015 & 0.30 & 130 \\
\hline TIR5 & 1074 & 0.13 & 42 \\
\hline M5R5 & 1220 & 0.23 & 8 \\
\hline M7R5 & 1288 & 0.18 & 51 \\
\hline F9R5 & 1288 & 0.24 & 14 \\
\hline F8R5 & 1324 & 0.20 & 140 \\
\hline T2R5 & 1368 & 0.12 & 33 \\
\hline M2R5 & 1380 & 0.19 & 53 \\
\hline F9R4 & 1470 & 0.16 & 34 \\
\hline M4R4 & 1518 & $0.13^{C}$ & 47 \\
\hline$M \perp R 4$ & 1606 & 0.18 & 37 \\
\hline M7R4 & 1647 & 0.16 & 54 \\
\hline M5R4 & 1659 & 0.26 & 54 \\
\hline M2R4 & 1884 & 0.24 & 14 \\
\hline $\mathrm{T} 15 \mathrm{R} 4$ & 2127 & 0.17 & 34 \\
\hline F9R3 & 2487 & $0.10^{\mathrm{C}}$ & 64 \\
\hline F8R3 & 2612 & $0.10^{\mathrm{C}}$ & 31 \\
\hline \multirow[t]{2}{*}{ M5R3 } & 2955 & $0.10^{\mathrm{C}}$ & 110 \\
\hline & & Average & $52 \pm 34^{\circ}$ \\
\hline
\end{tabular}

$\mathrm{a}_{(M)}=$ Measured fractional whole body retention.

${ }^{\mathrm{b}_{\text {(LKS })}}=\begin{aligned} & \text { Measured percent retention found in the liver, kidneys and } \\ & \text { spleen. }\end{aligned}$

Whole body retention estimated from previously published equation for $\mathrm{Ra}^{226}$ retention in the beagle (8) .

$\mathrm{d}_{\text {Mean }} \pm$ standard deviation of tabulated values. 
calcium concentration, which has been published(10), and the size of the three tissues of concern in the standard man. These data are summarized in Table 4 and are compared with the radium and strontium soft tissue studies on dogs in Table 5 .

Table 4. Comparison of Certain Soft Tissue to Whole Body Concentrations of Calcium in the Human

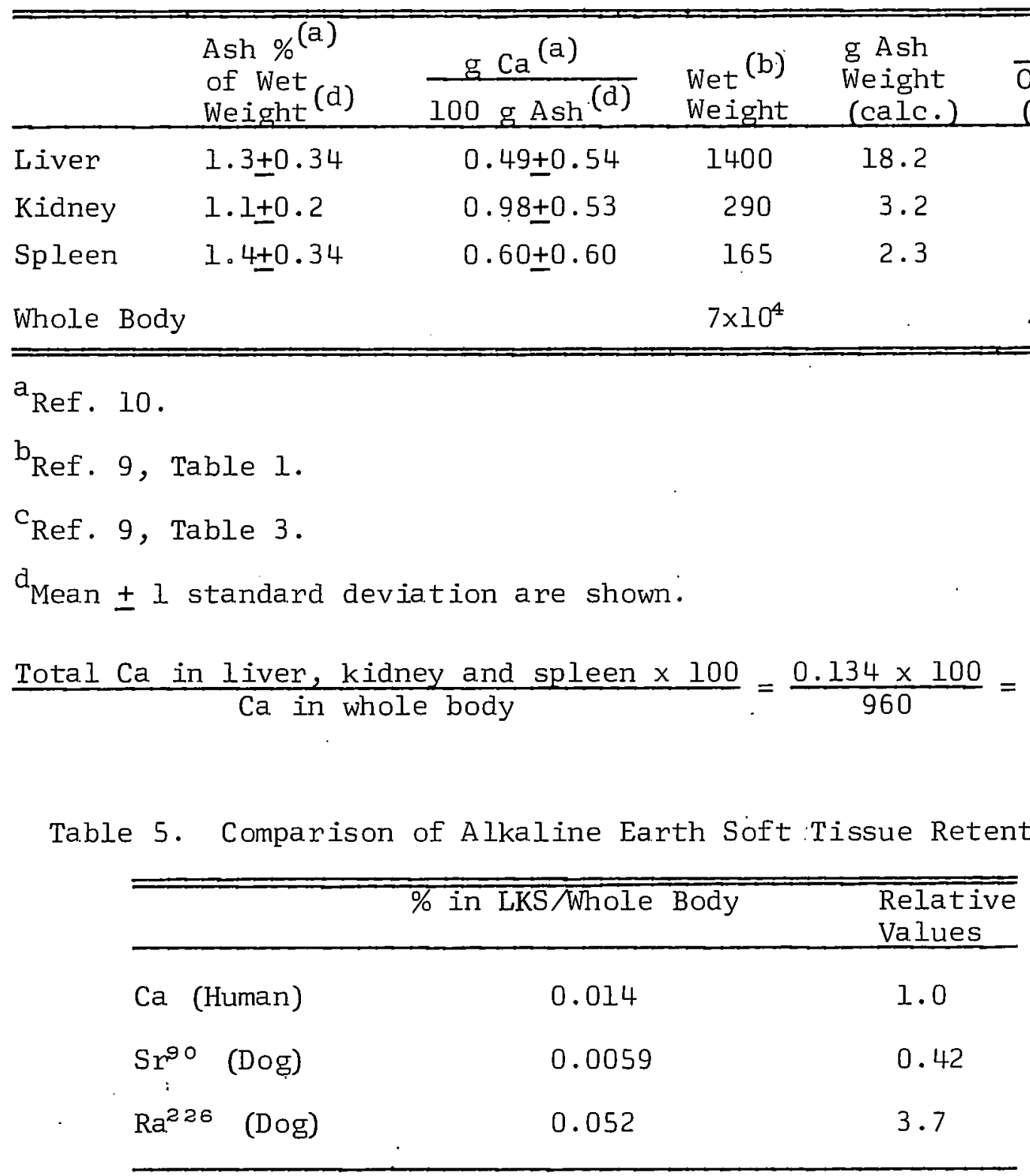




\section{References}

1. Betsy J. Stover and David R. Atherton, Metabolism of $\mathbf{S}^{90}$ in adult beagle dogs. Proc. Soc. Exptl. Biol. Med. 99, 201-205: (1958).

2. B. J. Stover, J. H. Dougherty, C. W. Mays and C. E. Rehfeld, Interim Report of Studies of $\mathrm{Sr}^{90}$ in Adult Beagles, Division of Radiobiology, Department of Anatomy, University of Utah, C00-221 (1960) .

3. Betsy J. Stover, David R. Atherton and Charles W. Mays, Studies of the retention and distribution of $\mathrm{Ra}^{226}, \mathrm{Pu}^{239}, \mathrm{Ra}^{228}\left(\mathrm{MsTh}_{1}\right)$, $\mathrm{Th}^{228}$ (RdTh), and $\mathrm{Sr}^{90}$ in adult beagles. In Some Aspects of Internal Irradiation (T. F. Dougherty, W. S. S. Jee, C. W. Mays and B. J. Stover, eds.) Pergamon Press (1962).

4. David R. Atherton, Betsy J. Stover, and C. W. Mays, Soft tissue retention of $\mathrm{Ra}^{225}$ and $\mathrm{Sr}^{90}$ given intravenously to the dog (abstract) . Radiation Research, 16, 4 (1962).

5. T. F. Dougherty, B. J. Stover, J. H. Dougherty, W. S. S. Jee, C. W. Mays, C. E. Rehfeld, W. R. Christensen, and H. C. Goldthorpe, Studies of the biological effects of $\mathrm{Ra}^{226}, \mathrm{Pu}^{239}, \mathrm{Ra}^{238}$ (MsTh $)$, $\mathrm{Th}^{228}$ (RdTh), and $\mathrm{Sr}^{\circ}$ in adult beagles. Radiation Research 17, 625-681 (1962) .

6. David R. Atherton, Betsy J. Stover and Charles W. Mays, Soft tissue retention of ${ }^{226} \mathrm{Ra}$. in the beagle. Health Physics 11, 101-108 (1965).

7. David R. Atherton, Determination of $\mathrm{Sr}^{90}$ in Biological Material with Solvent Extraction of $Y^{90}$ into Scintillator Solution, Research in Radiobiology, Report C00-119-233, p. 88 (1965) .

8. Thomas F. Dougherty, Research in Radiobiology, C00-119-232, p. 8 (1965)

9. Preliminary report of the ICRP Task Group on the Revision of the Standard Man (Provided by Walter S. Snyder and Mary Jane Cook).

10. Isabelle H. Tipton and M. J. Cook, Trace Elements in Human Tissue Part II. Adult Subjects from the United States, Health Physics $\underline{9}$, 103-145 (1963). 


\title{
DETERMINAT ION OF Sr 90 IN_B.IOLOG.ICAL_MA.TER.IAL_ WITH SOLVENT EXTRACTION OF YO INTO SCINTILLATOR SOLUT ION
}

\author{
David R. Atherton
}

\begin{abstract}
The general method of EHPA extraction of yttrium has been adapted for the analysis of excreta, bone and soft tissue samples containing $\mathrm{Sr}^{90}$. The feature of interest in the method is that the toluene used for dilution of. the EHPA contains PPO and POPOP which enables one to obtain count rates following extraction of the yttrium without further manipulation of the sample. Limit of the method is probably imposed by the counting system which in this laboratory permits the detection of $16 \mathrm{pC} i$ of $\mathrm{Y}^{\circ} \mathrm{w}$ with an expected error of $\pm 5 \%$ using 100 minute counting times.
\end{abstract}

\section{Introduction}

Many of the important characteristics of di(2-ethylhexyl)phosphoric acid (EHPA) have been described by Peppard et al. $(1,2)$. Petrow $(3,4)$ has described its use in methods used for the evaluation of $\mathrm{Ra}^{228}, \mathrm{Th}^{228}$ and $\mathrm{Sr}^{90}$ content of biological material, while Kauffman and Matuszek (5) have described a method for its use in determining the $\mathrm{Sr}^{90}$ content of water.

A method of determining the $\mathrm{Sr}^{90}$ content of dog excreta, bone and soft tissue was developed in this laboratory and is practically the same as that described by Kauffman and Matuszek (5). Basically, the procedure is to extract $y^{\circ}$ from the specimen of interest into a $1: 1$ dilution of EHPA in toluene which contains 2,5 dipenyloxazole (PPO) and 1,4 bis-2(5 phenyloxazolyl) benzene (POPOP). The organic phase is transferred to a $50 \mathrm{ml}$ Erlenmeyer flask and a count rate from the decay of $\mathrm{Y}^{\circ 0}$ is obtained by placing the flask on a photomultiplier tube which, of course, is associated with the proper electronic gear.

Because, in this system, analysis of samples of biological material present problems not encountered in the analysis of water samples, it is felt that a brief recitation of the method and a short discussion of two problems, referred to above, could be useful. 


\section{Experimental}

Apparatus. A photomultiplier tube (2 or 3 inch) in a light-tight compartment large enough to accommodate a $50 \mathrm{ml}$ Erlenmeyer flask. It is preferable that the volume containing the flask be shielded with about 2" of lead. The photomultiplier tube is properly connected to power supply, preamplifier, amplifier, scaler and timer.

Reagents. Nitric Acid, 30\% hydrogen peroxide, hydrochloric acid, di(2-ethylhexyl)phosphoric acid (EHPA), toluene, 2,5 dipenyloxazole (PPO), 1,4 bis-2-(5 phenyloxazolyl) benzene (POPOP), and absolute alcohol.

EHPA is prepared for use by diluting with an equal volume of toluene containing 8 gm PPO per liter and 0.2 gm POPOP per liter*.

Standard scintilator fluid is prepared by adding 4 gm PPO and 0.1 gm POPOP to a liter of toluene.

Procedure. Bone and fecal samples are prepared by incineration followed by solution in $\mathrm{HCl}$. Urine and soft tissue are wet ashed with heat, concentrated $\mathrm{HNO}_{3}$, and hydrogen peroxide, taken to dryness and put into $\mathrm{HCl}$ solution.

The $\mathrm{pH}$ of all samples is adjusted to 1.0 and $25 \mathrm{ml}$ of the EHPAscintillator solution are added to up to $100 \mathrm{ml}$ of sample solution. Samples are shaken 5 minutes (the time being noted so that a correction for decay of $Y^{\rho \circ}$ may be made) and at least 15 minutes are allowed for phase separation. The aqueous phase is drawn off and may be reserved for subsequent $\mathrm{Y}^{90}$ extraction (or other analysis) or discarded. The organic phase is washed with $25 \mathrm{ml}$ of $0.1 \mathrm{~N} \mathrm{HCl}$, and, after phase separation, the aqueous phase is disposed of as the first. The organic phase

\footnotetext{
* Some EHPA requires considerable pretreatment (3) to strip impurities from it. If pretreatment is necessary it might be well to add the PPO and POPOP after that procedure.
} 
is transferred into a $50 \mathrm{ml}$ Erlenmeyer flask, the separatory funnel is rinsed with $15 \mathrm{ml}$ of standard scintillator fluid and added to the sample and finally $10 \mathrm{ml}$ of absolute ethyl alcohol are added. The alcohol effects clearing of the sample which, unless prolonged times are allowed for phase separations, is an emulsion of the scintillator-extraction mixture with a trace of water.

The remaining step of the procedure is the evaluation of the $\mathrm{Y}^{\circ}$ content of the sample which is done by observing the relative count rates of the sample and a standard which is prepared in the following way. A known amount of $\mathrm{Sr}^{90}-\mathrm{Y}^{\circ \circ}$ contained in $1 \mathrm{ml}$ of a $0.1 \mathrm{~N} \mathrm{HCl}$ solution is placed in the same kind of flask as described above for the sample. 25 $\mathrm{ml}$ of the EHPA-scintillator mixture, $15 \mathrm{ml}$ of standard scintillator solution and $10 \mathrm{ml}$ of absolute alcohol are added. After vigorous shaking for a moment a clear standard of $\mathrm{Sr}^{\circ 0}-\mathrm{Y}^{\circ 0}$ results. Closing the flask tightly' insures a permanent standard.

Counting is done, as described by Kauffman and Matuszek (5), such that only the light flashes resulting from the $\beta$-decay of $Y^{\circ \circ}$ are detected.

\section{Results}

Typical results for three kinds of biological samples are shown in Table I. No effort has been made to test the limit of the sample size. The sample sizes tested were chosen on practical grounds--they are the sample sizes of interest.

The counting equipment consists of the bare essentials to do this kind of counting and 0.40 counts per $Y^{\ominus \circ}$ disintegration are observed with a blank* count rate of $20 \mathrm{c} / \mathrm{m}$. This places the sensitivity of the 
counting system such that, using 100 minute counting intervals, about 16 pCi of $\mathrm{Y}^{\circ \circ}$ can be detected with an expected counting error of $\pm 5 \%$.

TABLE.. I

Yield of Y९o with EHPA-Scintillator System

\begin{tabular}{|c|c|c|c|}
\hline Material & Amount & & $\begin{array}{l}\text { Fractional } \\
\text { Recovery }\end{array}$ \\
\hline Bone Ash & $0.392 \mathrm{~g}$ & & 0.975 \\
\hline Bone Ash & $1.176 \mathrm{~g}$ & & 1.01 \\
\hline Bone Ash & $1.960 \mathrm{~g}$ & & 0.980 \\
\hline Urine & $\sim 400 \mathrm{ml}$ & & 1.04 \\
\hline Feces & $1 / 10$ of 4 -day collection & & 0.978 \\
\hline \multirow[t]{2}{*}{ Feces } & $1 / 10$ of 4-day collection & & 0.950 \\
\hline & Average & 0.989 & \pm 0.03 \\
\hline
\end{tabular}

\section{Discussion}

The procedure for extracting $\mathrm{Y}^{90}$ and evaluating samples of biological material is quantitative, simple, and fast. There are two points of importance relative to the preparation of these materials for extraction which need mentioning:

First, the sample to be extracted must NOT be yellow. Yellow samples result from incomplete ashing and/or the presence of iron and in either case the colored material is extracted along with $\mathrm{Y}^{90}$ and a quenching. effect in the scintillator is observed. Incomplete ashing is most often encountered when wet ashing with concentrated $\mathrm{HNO}_{3}$ is employed. It is our experience that wet ashing can be completed somewhat more rapidly if the sample is taken to dryness in conc. $\mathrm{HNO}_{3} 3$ or 4 times, moistened with $30 \% \mathrm{H}_{2} \mathrm{O}_{2}$ and, on a hotplate, is taken to dryness repeatedly until no. charring occurs on drying. The sample is then wet with conc. HCl and diluted to the desired volume with water. 
If the sample contains enough iron to produce, a yellow color (red blood cells, for example) the iron can be removed by extraction with $30 \%$ Aliquat $336^{*}$ in toluene from $9 \mathrm{~N} \mathrm{HCl}$ solution of the sample, as described by Petrow (4), followed by readjustment of volume and pH for the EHPA extraction. An alternate and simpler method is to do an EHPA extraction as described above, discarding the organic phase. In either case the sample must be stored for an appropriate period for $Y^{\circ \circ}$ growth before an extraction for the evaluation of the $\mathrm{Sr}^{90}$ content of the sample can be done.

Storage of samples ready for extraction is the second point referred to above. It has been the experience of this laboratory that when samples are prepared for extraction, including adjustment of $\mathrm{pH}$ to 1.0 , that the $\mathrm{Y}^{\circ \circ}$ is not transferred quantitatively from the storage container to the extraction funnel even though three rinses of the storage container with $0.1 \mathrm{~N} \mathrm{HCl}$ were included in the procedure. Storage in either glass or plastic containers resulted in the loss of 40 , to $65 \%$ of the yttrium. It was also found that a single, short (10 second) rinse of the container with the EHPA-scintillator mixture would recover the adsorbed yttrium. Inasmuch as it is hardly feasible to store samples in separatory funnels, samples are prepared for extraction, stored in 8 ounce screw cap prescription bottles and, when the extraction is to.be done, the EHPAscintillator is added and shaking is done in the bottles. The emulsion is transferred to a separatory funnel; after phase separation, the bottle is rinsed into the funnel with $25 \mathrm{ml} 0.1 \mathrm{~N} \mathrm{HCl}$. The bottle is next rinsed with $15 \mathrm{ml}$ of the standard scintillator fluid which is trans-: ferred to the funnel for the last step in the procedure described above.

Methyltricaprylammonium chloride, available from General Mills Corp. 


\section{References}

1. D. F. Peppard, G. W. Mason, J. L. Maier, and W. J. Driscoll, Fractional Extraction of the Lanthanides as Their Di-Alkyl Orthophosphates. J. Inorg. Nucl. Chem. 4 , 33.4-343 (1957) .

2. D. F. Peppard, G. W. Mason, and S. W. Moline, The Use of Dioctyl Phosphoric Acid Extraction in the Isolation of Carrier-Free $\mathrm{Y}^{90}$, $\mathrm{La}^{140}, \mathrm{Ce}^{144}, \mathrm{Pr}^{143}$, and $\mathrm{Pr}^{144}$. J. Inorg. Nucl. Chem. $\underline{5}, 141-146$, (1957) •

3. Henry G. Petrow, Arthur Cover, Walter Schiessle, and Elizabeth Parsons, Radiochemical Determination of Radium-228 and Thorium-228 in Biological and Mineral Samples: Anal. Chem. 36, 1600-1603, (1964).

4. Henry G. Petrow, Rapid Determination of Strontium-90 in Bone Ash Via Solvent Extraction of Yttrium-90. Anal. Chem. 37, 584-586, (1965).

5. P. E. Kauffman, J. M. Matuszek, A Rapid Method for Sroo Analysis, Ninth Annual Meeting of the Health Physics Society, p/137, Cincinnati, Ohio, June 14-18, 1964. 
PREPARATION OF ST SO STANDÁRD IN PLASTIC BLOCK
FOR DENS ITOMETR IC EVALUATION OF RAD IOAUTOGRAMS

D. R. Atherton and W. S. S. Jee

Introduction

At the request of the Bone Group, the preparation of a standard for the evaluation of radioautograms produced by exposure: of film to tissue containịng $\mathrm{Sr}^{90}$ was undertaken.

The material specified for the standard was a block of bioplastic*. This material will set and harden when it contains a small amount of water but it is doubtful that a uniform distribution of strontium could be effected if it were introduced in an aqueous medium.

Recently, in working on the extraction of $Y^{90}$ (1) from $\mathrm{S}^{90}$ with EHPA, di (2-ethylhexyl) phosphoric acid), it was noted that the organic (EHPA) phase contained increasing amounts of $\mathrm{Sr}^{\circ 0}$ as the $\mathrm{pH}$ of the aqueous phase exceeded $\mathrm{pH} 2$. It was therefore decided to try to extract the $\mathrm{Sr}^{90}$ with EHPA at a fairly high $\mathrm{pH}$ and then add the $\mathrm{Sr}^{\circ \circ}$ contained in the organic phase to the bioplastic. The hope, of course, was that the two organic materials would be miscible and thus produce a uniform distribution of $\mathrm{Sr}^{90}$ in the finished bioplastic block.

\section{Procedure}

A 1:1 mixture of EHPA and toluene was shaken with an equal volume of a saturated solution of $\left(\mathrm{NH}_{4}\right)_{2} \mathrm{CO}_{3}$. Following phase separation, which was facilitated by centrifugation, the organic phase was washed with an equal volume of water and the organic phase retained.

The desired amount of $\mathrm{Sr}^{90}$ in dilute nitric acid solution was taken and the $\mathrm{pH}$ of the solution was raised to $\mathrm{pH} 7$ to 8 by dropwise addition

*Ward's Natural Science Establishment, Inc. 
of $5 \mathrm{~N} \mathrm{NaOH}$. The $\left(\mathrm{NH}_{4}\right)_{2} \mathrm{CO}_{3}$-treated EHPA, described above, was added and the two solutions shaken. Following phase separation, duplicate aliquots of the organic phase were added to 1. Bioplastic and 2. a. volumetric flask.

The bioplastic and EHPA were mixed well both prior to and following the addition of the catalyst used to promote the polymerization of the bioplastic. The liquid bioplastic was weighed, placed in a chamber with a somewhat reduced pressure until entrapped gas was evolved, and then placed in a drying oven at about $100^{\circ} \mathrm{C}$ for 60 hours. After removal from the oven the block was kept at room temperature and weighed each day for two days at which time it appeared to have attained a constant weight.

The aliquot placed in the volumetric flask was shaken with several times its volume of $6 \mathrm{~N} \mathrm{HCl}$ to strip the $\mathrm{Sr}^{\circ}$ from the EHPA and the volume was adjusted to the mark with $0.1 \mathrm{~N} \mathrm{HCl}$. An aliquot of the aqueous phase of this material was evaluated for its $\mathrm{Sr}^{90}$ content in order that the $\mathrm{Sr}^{\circ 0}$ concentration of the bioplastic block could be calculated.

Volume ratios used were as follows:

$$
\begin{aligned}
& \mathrm{Sr}^{\circ \circ} \text { at } \mathrm{pH} 7 \text { to } \mathrm{EHPA}=2: 1 \\
& \mathrm{Sr}^{\circ} \mathrm{-EHPA} \text { to bioplastic }-1: 4
\end{aligned}
$$

\section{Results}

$\mathrm{Y}^{\circ}$ was not extracted into the EHPA under these conditions, the high $\mathrm{pH}$ probably caused it to adsorb onto the surface of the container.

$\mathrm{Sr}^{90}$ appeared to have been handled quantitatively in the system.

A preliminary report by the Bone Group indicates that the distribution of the $\mathrm{Sr}^{\circ}$ in the bioplastic block is satisfactorily uniform. 


\section{References}

1. David R. Atherton, Determination of $\mathrm{Sr}^{90}$ in biological material with solvent extraction of $\mathrm{Y}^{90}$ into scintillator solution, page 88 of this report. 


\title{
GAMMA-RAY EMITTERS IN CONCRETE
}

Ray D. Lloyd, Gerald B. Folland, Bruce W. Church, and David H. Taysum

\begin{abstract}
The content of radionuclides in samples of readymix concrete obtained from three local suppliers was determined by gamma-ray counting. Average concentration of gamma emitters in the concrete in $\mathrm{pCi}$ per gram was found to be: $\mathrm{Ra}^{226} 0.7$; $\mathrm{Ra}^{228} 0.6$; $\mathrm{Th}^{228} 0.6$; and $\mathrm{K}^{40} 7.9$ (9 mg $\mathrm{K}$ ). Retention of $\mathrm{Rn}^{222}$ activity in the hardened concrete was about $85 \%$ that of the $\mathrm{Ra}^{226}$. The radioactivity of constituents was also measured. Concentration of gamma-ray emitters in gravel was approximately equal to that in the concrete mix, while their concentration was about $40 \%$ higher in sand and about $50 \%$ lower in cement.
\end{abstract}

\section{Introduction}

Some contribution to the gamma-ray background is made by radioactivity in construction materials. A study of the gamma-ray activity of concrete was undertaken to select materials for the erection of our new low background counting facility.

\section{Materials and Methods}

Samples were collected for evaluation in steel cans $8.2 \mathrm{~cm}$ diameter $x 11 \mathrm{~cm}$ high (\#2 size) from three major suppliers of ready-mix concrete in the Salt Lake area. These suppliers were A. J. Dean and Sons Ready Mix, 3041 East 3300 South; Utah Ready Mix Concrete Co., 1730 Beck Street; and Dan R. Fogle Red-E-Mix; 337 Hartwell Avenue. Enough sand, gravel, cement and actual concrete (mix) was collected so that each type of sample from each supplier filled two \#2 cans. Pieces of hardened mix were collected from Dean, while wet mix was obtained from Utah and Fogle. Other samples were dry when put into the cans.

All three companies obtain their cement from two suppliers, UtahPortland Cement Co. (from Parley's Canyon near Salt Lake City) and Ideal Cement Co. (near Morgan, Utah) . Our cement samples contained only UtahPortland except for the Utah Ready Mix Co. sample, which was half UtahPortland and half Ideal. 
Sand and gravel are obtained from company excavations along the Wasatch Front, near Salt Lake City, in shoreline deposits of ancient Lake Bonneville, which was contemporary with the Pleistocene glacial period. An attempt was made to obtain as representative a sample of sand and gravel as possible from each supplier. However, the sand and gravel deposits are not uniform throughout, and the composition of excavated material may change as different sections of the deposit are removed for use.

Counting was done with an $8 \times 4$ inch NaI(Tl) crystal connected to a 400 channel pulse-height analyzer. A specially constructed plastic positioner held the 2 -can samples at a standard $1.5 \mathrm{~cm}$ distance from the crystal face. Sample and detector were enclosed by a steel chamber with 6 inch thick walls. Sixty minute counting periods were used. The cans were initially counted unsealed after the wet concrete mix had hardened, then all cans were sealed against radon leak and recounted after a three week interval to allow the $\mathrm{Rn}^{2 a 2}$ to build into secular equilibrium with its parent $\mathrm{Ra}^{226}$.

Standards in glass ampoules $(10 \mathrm{ml})$ were counted in \#2 cans containing an absorber similar in density to each type of sample. A well in the center of the absorber was provided for the ampoule. The relative counting rates of an ampoule source centered in a can and an equal activity distributed throughout a can at this distance from our crystal was determined. Glass ampoules containing $\mathrm{Zr}^{95}+\mathrm{Nb}^{95}(\gamma$-ray energy $0.76 \mathrm{Mev})$ and $\mathrm{K}^{40}(1.46 \mathrm{Mev})$ were counted in the center well of a unit density absorber in a \#2 can. Their contents were then dissolved in individual \#2 cans of water and recounted. The ratio $\left[\frac{\mathrm{cpm} \text { as ampoule in well }}{\mathrm{cpm} \text { as distributed source }}\right]$ for the moderate energy $\mathrm{Zr}^{95}+\mathrm{Nb}^{95}$ was 0.906 and for the high energy $K^{40}$ was 0.915 , averaging 0.91 . It was 
therefore assumed that this ratio is relatively independent of the photon energies considered and this ratio was used to correct the counting rates of all ampoule sources used as:standards.

Results and Discussion

Table I summarizes the results of this study. Gamma-ray emitters which were identified in each of the samples were $\mathrm{Ra}^{226}$ (via $\mathrm{Bi}^{214}$ at $1.76 \mathrm{Mev}$ ), $\mathrm{Ra}^{228}$ (via $\mathrm{Ac}^{228}$ at $0.93 \mathrm{Mev}$ ), $\mathrm{Th}^{228}$ (via $\mathrm{Tl}^{208}$ at $2.62 \mathrm{Mev}$ ) and $\mathrm{K}^{40}$ (1.46 Mev). In each energy band, the contribution of other emitters was stripped out. Concentration of these emitters in the concrete samples (in $\mathrm{pCi} / \mathrm{kg}$ ) averaged; $\mathrm{Ra}^{226} 738 ; \mathrm{Ra}^{228}$ 618; $\mathrm{Th}^{228}$. 555 ; and $\mathrm{K}^{40} 7929$ (9.35 gm K). Indïvidual values were all within about $\pm 20 \%$ of the averages. Unsealed concrete samples retained about $85 \%$ of the $\mathrm{Rn}^{222}$ being produced by the radioactive decay of $\mathrm{Ra}^{226}$. Average concentration of these gamma ray emitters in the gravel samples was roughly equal to that in the mix, while the concentration in sand was about $40 \%$ higher, and in cement it was about $50 \%$ lower. 
TABLE I

Concentration of Gamma-ray Emitters

in Concrete and its Constituents.

\begin{tabular}{|c|c|c|c|c|c|c|c|}
\hline Sample & $\begin{array}{l}\text { Weight } \\
\text { kg }\end{array}$ & $\frac{\mathrm{gm} \mathrm{K}}{\mathrm{kg}}$ & $\frac{\mathrm{pCi} \mathrm{Ra}^{228}}{\mathrm{~kg}}$ & $\frac{\mathrm{pCi} \mathrm{Th}^{228}}{\mathrm{~kg}}$ & $\frac{\mathrm{pCi} \mathrm{Ra}^{226}}{\mathrm{~kg}}$ & in & $\begin{array}{l}\frac{\mathrm{Rn}^{222}}{\mathrm{Ra}^{226}} \\
\text { Concrete }\end{array}$ \\
\hline MIX & & & & & & & . \\
\hline Dean & 0.890 & .10 .88 & 678 & 636 & 930 & $\because$ & 0.73 \\
\hline Utah & 2.670 & 7.82 & 565 & 491 & 609 & & 0.96 \\
\hline Fogle & 2.726 & 9.36 & 610 & 538 & 674 & & 0.87 \\
\hline & Average & 9.35 & 618 & 555 & 738 & & 0.85 \\
\hline
\end{tabular}

$\underline{\text { SAND }}$

Dean

$1.538 \quad 13.40$

700

734.

895

Utah

i.938 11.88

664

683

842

Fogle

$\begin{array}{lll}1.344 & 20.56 & 1018 \\ \text { Average } & 15.28 & 794\end{array}$

1018

$\underline{1298}$

Average 15.28

812

1012

CEMENT

$\begin{array}{lllllll}\text { Dean } & 1.334 & 3.08 & 292 & 237 & 439 \\ \text { Utah } & 1.292 & 4.10 & 334 & 366 & 597 \\ \text { Fogle } & 1.287 & \underline{2.49} & \underline{294} & \underline{262} & \underline{504} \\ & \text { Average } & 3.22 & 307 & 288 & 513\end{array}$

GRAVEL

$\begin{array}{llllll}\text { Dean } & 1.544 & 12.50 & 553 & 567 & 844 \\ \text { Utah } & 1.546 & 13.46 & 783 & 752 & 857 \\ \text { Fogle } & 1.600 & \underline{8.05} & \underline{326} & \underline{339} & \underline{500} \\ & \text { Average } & 11.34 & 554 & 553 & 734\end{array}$




\section{USE OF THE CHINCH ILLA \\ IN THE STUDY OF BONE SEEKING RADIONUCLIDES \\ G. N. Taylor, L. Brewster, C. W. Mays and S. Orme}

Abstract: A brief evaluation of the suitability of the chinchilla as an experimental animal for toxicity studies dealing with several bone-seeking radionuclides is being made. This species has a maximum life span of 20 to 30 years, an epiphyseal closure time of 800 to 1000 days, and a skeletal to body weight ratio of approximately .07 . The injected $\mathrm{LD}_{50-30}$ dose for $\mathrm{Ra}^{226}$, given in isotonic saline via a single subcutaneous injection is about $800 \mu \mathrm{Ci} \mathrm{Ra}^{226} / \mathrm{kg}$.

A survey concerning the suitability of the chinchilla Chinchilla langigera) for use in the study of some of the bone-seeking radionuclides is being made. This paper will present a few of the features that have been investigated thus far.

Three of the factors that prompted our interest in this species were the relatively long life expectancy, as indicated by some reports(1), its desirable husbandry factors, and the economy of its maintenence.

Some disagreement was found relative to longevity and to more definitely establish this parameter we mailed questionaires to 30 recognized chinchilla ranchers in various parts of the $U$. S. and in Canada and England. Each was asked to indicate(I) the oldest chinchilla he had ever owned; (2) the oldest he had ever heard of; and (3) to estimate the average life span. The last item, average life span, proved to be an impractical question since such a high percentage are eventually pelted and not allowed to die a natural death. Replies to the other two questions ranged as follows:

$$
\begin{array}{lr}
\text { Oldest owned: } & 7 \text { to } 21 \text { years } \\
\text { Oldest heard of } & 12 \text { to } 27 \text { years }
\end{array}
$$

These data substantiate the relatively long life span and indicate the maximum to be in excess of 20 years and comparable to the dog. Thus this species could be used in some instances to compare rodent and canine 
studies without life expectancy as a variable.

Another factor investigated was the closure time of the epiphyses. This was estimated by radiographic examination of partially defleshed carcasses ranging from 6 months to 5 years at the time of death and from histologic study of the proximal tibial and the distal ulnar and radial epiphyses. Based on these findings closure occurs in the appendicular skeleton between 800-1000 days - significantly later than in the dog. The skeleton of this species further differed from the dog by the absence of typical osteones.

Thus far, organ weights have been determined in only one animal. These are summarized in Table 1. A point of interest is the relatively large adrenal gland as reported in other studies(2). In determining the skeletal weight, the carcass was kept moist with isotonic saline during defleshing and was weighed in a damp condition. The marrow was not removed.

Table 1. Organ and Skeletal Weights. (total body weight 518.35 gms; 17 months age)

\begin{tabular}{lcll}
\hline Heart & (gms) & Testes (both) & (gms) \\
Lungs & 1.795 & . & 4.851 \\
Liver & 2.333 & Adrenals (both) & 0.105 \\
Spleen. & 16.233 & Pituitary & 0.010 \\
Kidneys (both) & 0.682 & Eyes (both) & 1.867 \\
Brain & 4.332 & Thymus & 0.642 \\
& 4.799 & Skeleton & 37.330
\end{tabular}

$\frac{\text { Weight of skeleton }}{\text { Total body weight }}=\frac{37.330}{518.35}=.072$

$\mathrm{Ra}^{226}$. toxicity studies using 20 chinchillas are in progress (Table 2) The dose levels have been arranged to elicit both acute and chronic injury, and a wide age span has been employed to observe the significance 
Table 2. Summary of Chinchillas Used in $\mathrm{Ra}^{226}$ Toxicity Study

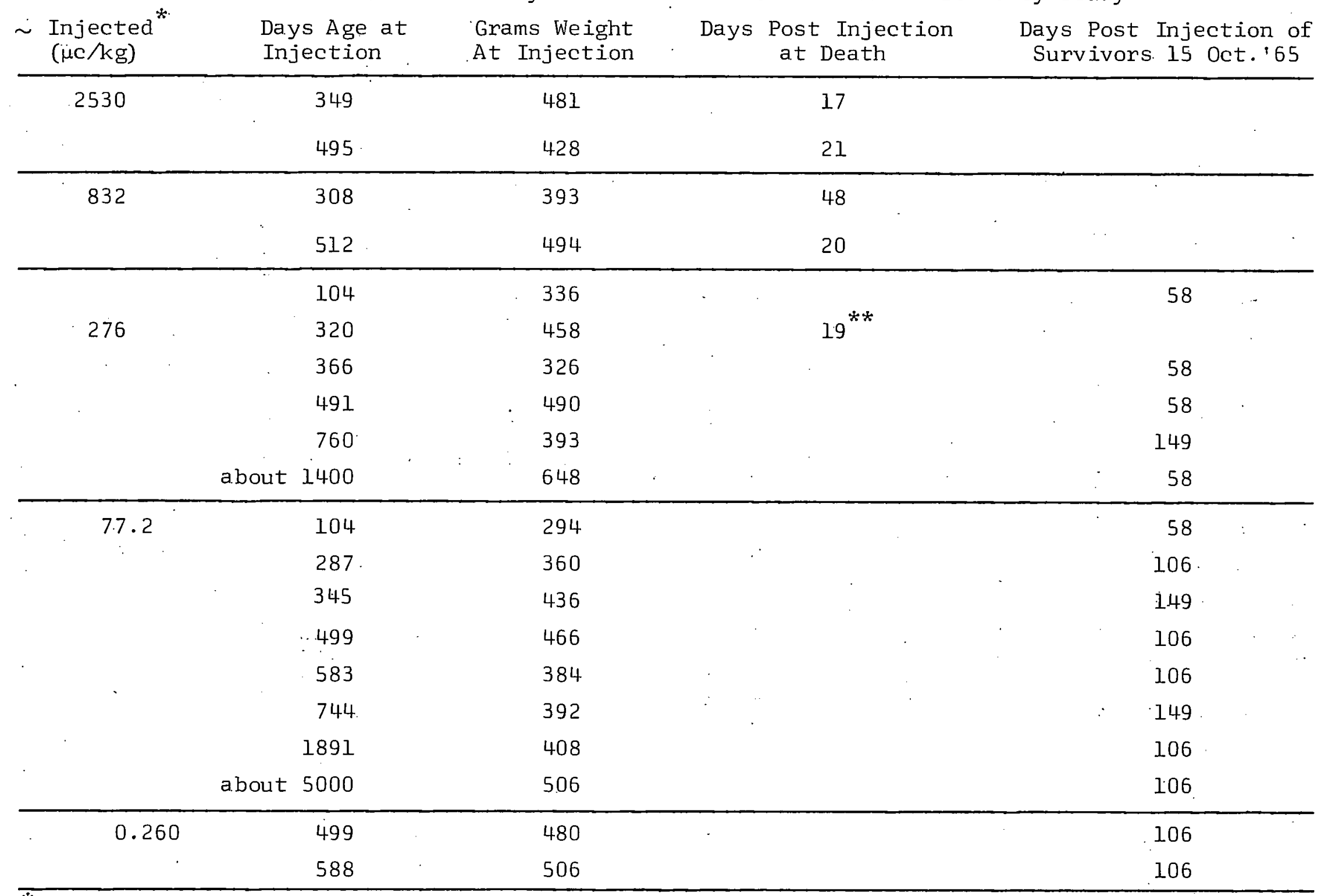

* Given in isotonic saline via a single subcutaneous injection.

** The immediate cause of death was not radiation induced. 
of age at injection. Administration of the nuclide was via a single subcutaneous injection in an isotonic saline solution. Early observations have indicated that the $\mathrm{LD}_{50-30}$ is approximately 800 injected $\mu \mathrm{c} / \mathrm{kg}$ of body weight (see Mays et al., this report for retention(3)) .

of the long term effects, osteosarcoma induction time should be especially interesting. If the latent period is related to life span, as suggested by Brues(4), this interval should be similar for a corresponding radiation exposure for both the dog and chinchilla.

\section{Acknowledgements}

Our appreciation is extended to Mr. Warren Lund and Mr. C. J. Trimble who were very helpful in supplying animals, and to the following chinchilla ranchers who assisted in approximating the life span: Mr. Harold Anderson, Atlantic, Iowa; Dr. F. E. Button, Sedalia, Colorado; Mr. Walter Campbell, North Haledon, New Jersey; Mr. Bill Capobianco, Catskill, New York; Corry Chinchilla Farm, Auburn, Iowa; Ready Chinchilla Farm, Bowling Green, Ohio; Mr. Fred J. Cox, Lexington, North Carolina; Mr. Bob Czapowski, Delafield, Wisconsin; Mr. E. B.'Finch, The Falls, Bass Lake, California; Mr. Irven C. Graham, Radcliff, Kentucky; Mr. Anthony Gujda, Swedesburg, Pennsylvania; Mr. Chapin Hand, Seward, Nebraska; Mr. Wayne G. Hendrickson, Santa Barbara, California; Mr. Austin Hollway, Centreville, Virginią; Mr. H. W. Hunt, Jr., Champaign, Illinois; Mr. Norbert Krause; Milwaukee 16, Wisconsin; Mr. W. F. Mays, Elizabethtown, Kentucky; Mr. J. A. Moore, De Soto, Texas; Mr. Frank Natole, Minneapolis, Minnesota; Miss Polly 0'Rosky, Harrisburg, Pennsylvania; Mr. Stan Pangborn, Washington 16, D. C.; Mr. James Patrick, New Paltz, New York; Mr. Walter Pohl, Hollywood 46, Caḷifornia; Mr. Edward Schroeder, Milwaukee 6, Wisconsin; Mr. Kurt Sonnenburg, Springfield, Virginia; Mr. Arthur T. Sweeney; Boston 24, Massachusetts; Mr. Wade Watson, Aledo, Texas; Mr. Joe B. Witt, Fairfield, Illinois; and Mr. Dick Wyatt, Sutherland, Nebraska. 


\section{References}

1. Empress Chinchilla 21 (No. 3): 7 (1965).

2. T. B. Roos and R. M. Shackelford, Some observations on gross anatomy of the genital system and two endocrine organs and body weights in the chinchilla, Anatomical Record, 123 (3):301-302 (1955).

3. C. W. Mays, R. D. Lloyd, G. N. Taylor, R. Stair, L. Brewster ànd D. R. Atherton, Radium retention in chinchillas, (This report):

4. A. M. Brues, Radiation as a carcinogenic agent, Rad. Res. 3 (No. 3): $272-280 \quad(1955)$. 
RADIUM RETENTION. IN CH INCHILLAS

Charles W. Mays, Ray D. Lloyd, Glenn N. Taylor, Richard Stair, Lynn Brewster and David R. Atherton

Abstract: Preliminary results suggest that the early retention of radium in the chinchilla is similar to that in man. This similarity, combined with the chinchilla's long life span and small space requirements may make "it an excellent animal for internal emitter RBE studies. Additional work is needed and is in progress.

PILOT STUDY

A limited number of chinchillas were obtained from local breeders. Most of these animals were fur chewers and thus could be obtained at negligible cost. $\mathrm{Ra}^{226}$ was injected subcutaneously in the back. Unfortunately, the first group was injected with radium in sodium-citrate, citric-acid solution ( $\mathrm{pH} 3.5$ and 0.08 molar in total citrate). This caused a high local retention of radium in soft tissue at the injection site; therefore, all animals in the first injection group were sacrificed. Subsequent groups were injected subcutaneously with radium in physiological saline solution; in which case the skeleton was the principal site of retention.

RADIUM RETENTION (total body)

$\mathrm{Ra}^{226}$ retention at death in several saline-injected chinchillas is shown in Fig. 1. These values supported by total body counting results for living chinchillas (not plotted) indicate that radium retention in the chinchilla is much lower than that in the dog or mouse, and in fact appears similar to that in man. Whether this similarity will persist at later times is being determined.

EXCRETION

About $94 \%$ of the excreted $\mathrm{Ra}^{226}$ is eliminated in the feces, and about $6 \%$ in the urine. Similar values occur in humans. The large clearance of radium by the digestive tract causes its low retention in man. The same 
may be true in the chinchilla. Blood clearance is being evaluated. RADIUM DISTRIBUT ION IN TISSUE

As shown below in Table 1, subcutaneous citrate injections resulted in a large deposition of $\mathrm{Ra}^{226}$ at the injection sites, whereas with saline, the skeleton contained nearly all of the" body burden. The exact" mechanism of this large soft tissue deposition following a sodium citrate, citric acid injection is unknown.

\section{TABLE 1. RADIUM DISTRIBUTION}

Subcutaneous injection as:

C ITRATE

SAL TNE

Days after injection:

$\frac{.72}{\text { CIR6 }} \quad \frac{48}{\text { ClR7 }}$

Chinchilla number:

Skeleton

Injection site

Other soft tissue

Total burden
$38 \%$

$18 \%$

$44 \%$

$100 \%$
$37 \%$

$17 \%$

$46 \%$

$100 \%$

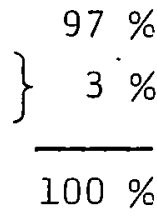

FRACTIONAL RADON RETENTION IN TISSUE

$\mathrm{Rn} / \mathrm{Ra}$ ratios were evaluated for the fresh tissues of ClR9. Unfortunately, in vivo values were not obtained for CIR7 and CIR6, but can be estimated from the $\mathrm{Rn} / \mathrm{Ra}$ in thawed, cut apart samples from these animals which were exposed to air several months after death. The $\mathrm{Rn} / \mathrm{Ra}$ ratio in living tissue is only slightly lower than in air-equilibrated dead tissue (see ClR9 femur and'tibia sample in Table 2).

In chinchilla bone, the $\mathrm{Rn} / \mathrm{Ra}$ ratio is similar to that for beagle bone at the same time after radium deposition, regardless of whether the radium was injected in saline or citrate. The high $\mathrm{Rn} / \mathrm{Ra}$ ratio in the pelt and injection sites is very interesting. It is also interesting that the $\mathrm{Rn} / \mathrm{Ra}$ ratio in chinchilla teeth is higher than that in chinchilla 
bone, since R. E. Rowland ${ }^{+}$has observed similar relationships in human teeth. In contrast, the $\mathrm{Rn} / \mathrm{Ra}$ ratio in the teeth of beagles injected intravenously with $\mathrm{Ra}^{226}$ in citrate as adults in only half the $\mathrm{Rn} / \mathrm{Ra}$ in their bones. ${ }^{++}$

TABLE 2. FRACTIONAL RADON RETENTION

$\mathrm{Rn} / \mathrm{Ra}$ Ratio

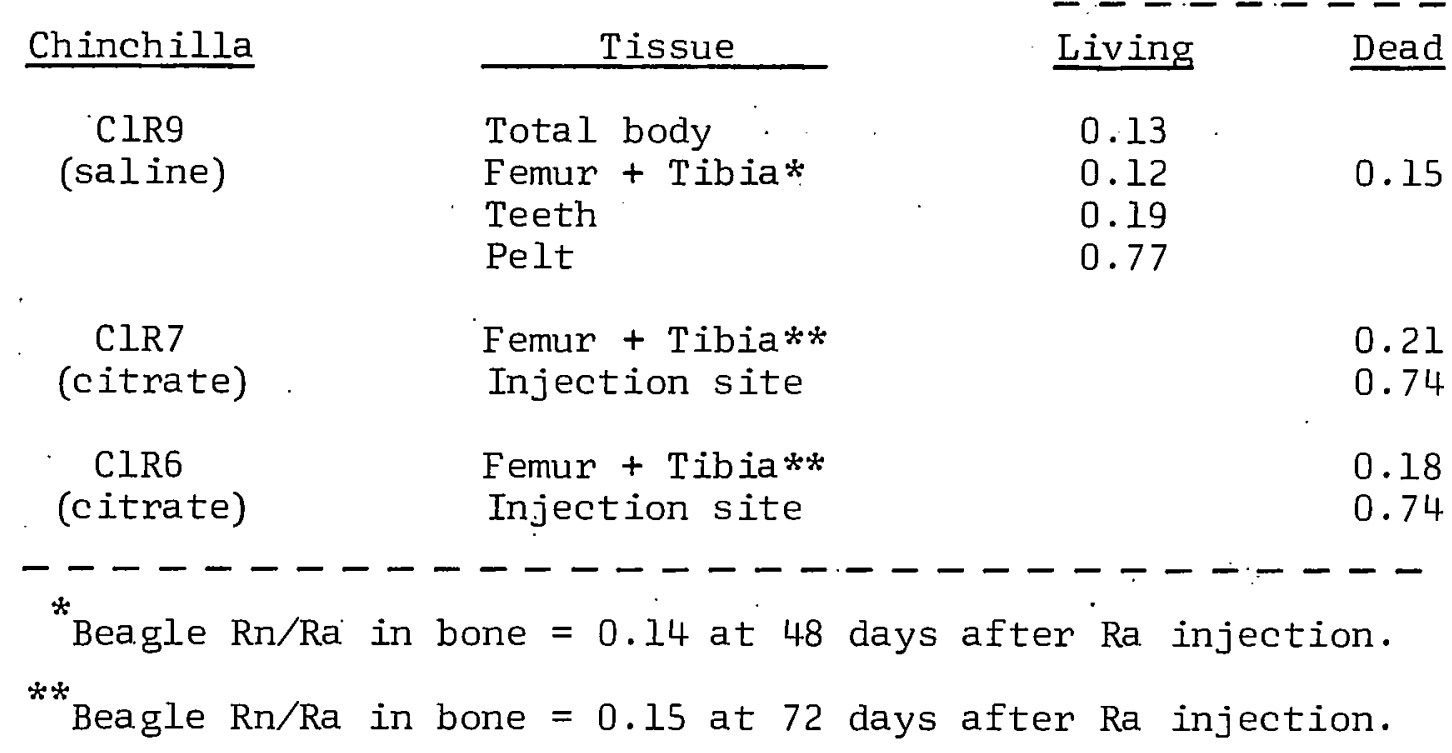

\section{SUMMARY}

(1) The metabolism of radium and radon is being studied in the chinchilla.

(2) Large soft tissue deposition of. radium occurred at the injection site when given subcutaneously in. citrate solution: this effect was absent with saline.

(3) The total body retention of radium in the chinchilla is low and is similar to that in man, at least for the first 100 days after injection.

(4) The primary route of radium excretion is fecal for the chinchilla, as it also is in man.

(5) The $\mathrm{Rn} / \mathrm{Ra}$ ratio in chinchilla bone is similar to that in other species at the same time after radium deposition.

(6) These preliminary results suggest that the chinchilla may be an excellent animal for internal emitter RBE studies.

$+$

Radium in Human Teeth. Arch. Oral Biol. 8:12-21, 1963.

${ }^{++}$Radon retention in radium-injected beagles, Rad. Res. 8:6, 480-489 (1958). 
RADIUM RETENTION IN SEVERAL SPECIES

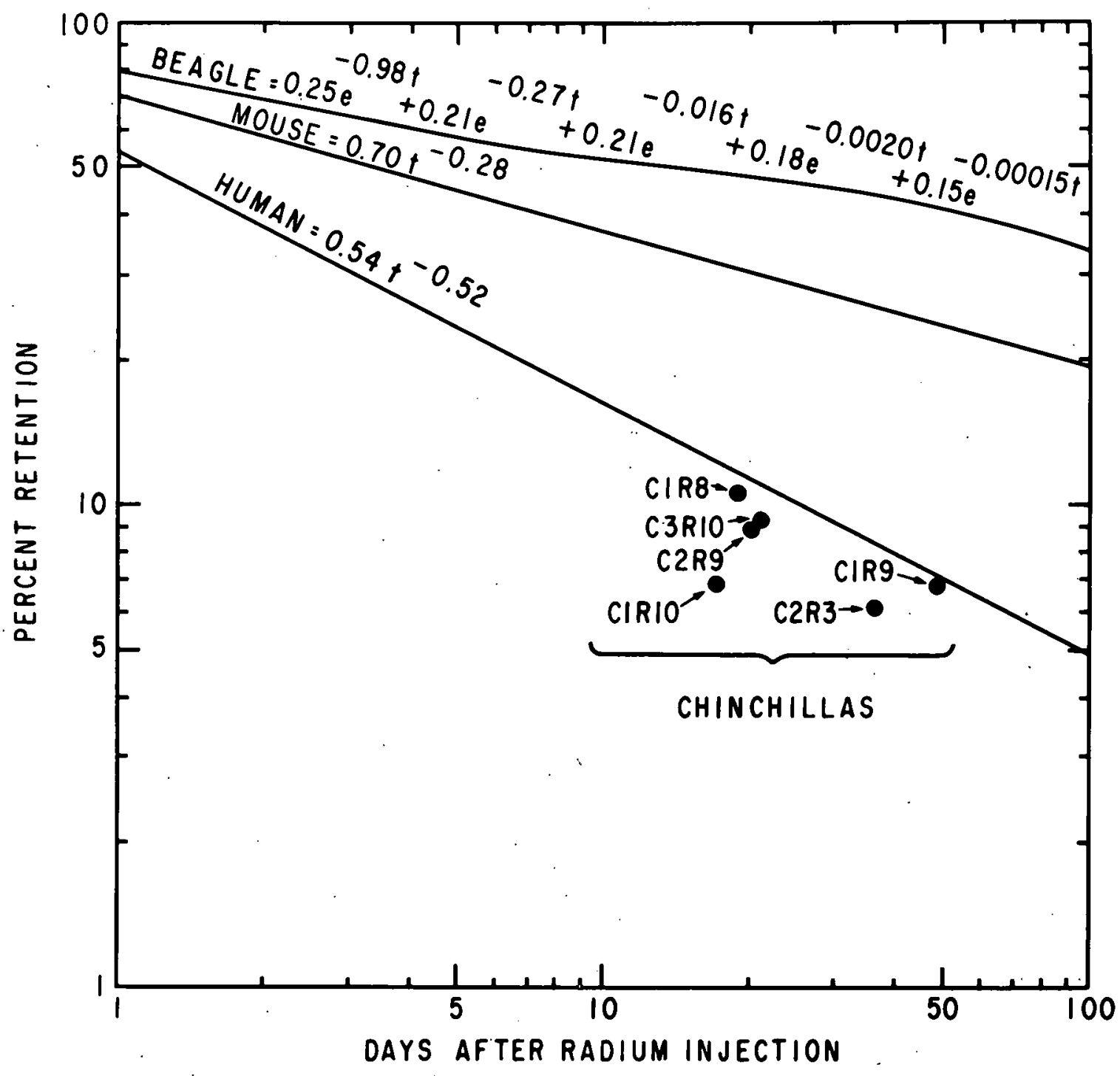

Fig. 1. Radium retention in several species. These chinchillas were injected subcutaneously with $\mathrm{Ra}^{226}$ in saline solution. 